\title{
5 Semantische Parameter von Konstruktionen
}

Das konstruktionssemantische Modell der drei Typen von Frames und Bedeutungen auf Type- und Token-Ebene, das ich in Kapitel 4 vorgestellt habe, ist ein erster Zugang, die semantischen Eigenschaften zu erfassen, die für eine Konstruktion und ihre Konstrukte eine Rolle spielen. Möchte man mehr von der semantischen Leistung einer Konstruktion erfahren und sie generalisiert über deren Konstrukte in einem konstruktikographischen Format beschreiben, ist es notwendig, die drei Typen von Frames und Bedeutungen miteinander in Beziehung zu setzen und ihr Zusammenspiel zu eruieren. Da dies zu einem großen Teil über die Feststellung der Frames auf Type- und Token-Ebene hinausgeht, möchte ich in diesem Kapitel als Basis für eine konstruktionssemantische Analyse im Allgemeinen und als konstruktikographische Analysekategorien im Besonderen sieben semantische Parameter von Konstruktionen vorschlagen. ${ }^{1}$

Die sieben Parameter dienen einerseits dazu, grundlegende Fragen der konstruktikographischen Erfassung von Konstruktionen hinsichtlich ihrer semantischen Eigenschaften zu klären und sind andererseits Gegenstand einer konstruk-

\footnotetext{
1 Den Begriff des Parameters nutzt bereits Lakoff (1987: 488-489) zur formalen und semantischen Beschreibung von Konstruktionen, wobei er für Letztere lediglich zwei Parameter ansetzt: die ,Bedeutungen' einzelner Strukturelemente der Konstruktion und die ,Bedeutung“ der Konstruktion als Ganzes. Langacker (2000: 24-28) setzt für die Regularität einer Konstruktion drei Parameter an: Kompositionalität, Spezifiziertheit und Produktivität, von denen ich die letzten beiden adaptiere. Ebenfalls unter dem Begriff des Parameters setzt Felfe (2012: 70-79) teilweise sich mit meinem Ansatz überschneidende Parameter zur Unterscheidung von syntaktischen Konstruktionen gegenüber LE an: formale Komplexität, semantische Allgemeinheit, Kompositionalität und Transparenz sowie Produktivität. Felfe verweist dabei auf Traugott (2008a: 8), die sich für die Parameter Allgemeinheit, Produktivität und Kompositionalität wiederum auf einen Vortrag Langackers beruft. Den Parameter der Kompositionalität setze ich nicht separat an, da Kompositionalität einerseits, wie in Unterabschnitt 2.2.1 argumentiert, zumindest für ArgumentstrukturKonstruktionen nicht auf Type-Ebene, sondern auf Token-Ebene (Konstruktebene) zu verorten ist. Andererseits beinhaltet der Parameter der emergenten Struktur (Abschnitt 5.7) ebensolche Aspekte von Nicht-Kompositionalität, weshalb ich sie im Zuge dieses Parameters diskutiere (vgl. schon Unterabschnitt 4.1.3). Weiterhin stellt Ziem (2018a: 8-11) eine Reihe von Untersuchungsgesichtspunkten von Phrasemen zusammen, von denen sich vier in den in diesem Kapitel vorgestellten Parametern wiederfinden: Musterhaftigkeit (hier: formale Abstraktheit), typische Filler der Leerstellen, Constraints (hier: Beschränkungen und Präferenzen) sowie Produktivität. Vier der fünf von Engelberg (2019: 16-22) zusammengestellten Eigenschaften von Argumentstrukturen überlappen sich ebenfalls mit meinen Parametern: Abstraktheit, Präferenzbasiertheit, Produktivität und Koerzion. Vgl. ferner auch Welke (2005: 71-91), der eine Reihe von Parametern für eine funktionalgrammatische Analyse aufstellt.
} 
tionssemantischen Analyse. Sie sollen in erster Linie folgende Fragen beantworten.

- Welche allgemeinen (formalen) Eigenschaften einer Konstruktion sind ausschlaggebend für ihre semantischen Eigenschaften und für die Evokation des Konstruktions-Frames?

- Welchen Einfluss haben Konstruktions-Frame und Konstruktionsbedeutung auf allgemeine konstruktikographische Aufgaben wie das Anlegen von Konstruktionseinträgen?

- Welche Rolle spielt die Instanziierbarkeit bestimmter Strukturelemente, insbesondere hinsichtlich der LE, die lexikalische Frames evozieren, für die semantischen Eigenschaften einer Konstruktion?

- Wie stark unterscheiden sich lexikalische Frames von einem KonstruktionsFrame und welche Konsequenzen hat der Grad dieser Unterscheidung für die Konstitution von Konstrukt-Frames?

- Wie groß ist der Einfluss des Konstruktions-Frames auf die Konstitution von Konstrukt-Frames - mit anderen Worten: Bis zu welchem Grad sind lexikalische Frames ausreichend, um Konstrukt-Frames zu konstituieren und wann tritt in einem Konstrukt Koerzion auf?

- Wie lässt sich die Produktivität einer Konstruktion auf Basis des konstruktionssemantischen Modells aus Kapitel 4 und damit genuin semantisch bestimmen?

- Wie sind semantische Aspekte, die außerhalb der Feststellung von lexikalischem Frame und Konstruktions-Frame liegen und auch über lexikalische Bedeutung und Konstruktionsbedeutung hinauszugehen scheinen, zu erfassen?

Ich erhebe nicht den Anspruch, dass es sich bei den sieben semantischen Parametern um eine vollständige Liste handelt und dass jeder Parameter für jede Art von Konstruktion gleichermaßen relevant ist. Vielmehr handelt es sich dabei um Aspekte, die sich bereits bei einer vergleichsweise kleinen Menge an Konstruktionen wie den dreien, die ich untersuche, als relevant erweisen. Folgende Parameter möchte ich unterscheiden:

1. formale Abstraktheit: die Komplexität der Konstruktion hinsichtlich der Anzahl ihrer Strukturelemente und ihrer lexikalischen Spezifiziertheit;

2. konstruktionelle Polysemie: die Frage, worauf unterschiedliche Lesarten von Konstrukten derselben Konstruktion zurückzuführen sind und welche Auswirkungen dies auf die konstruktikographische Erfassung hat;

3. Beschränkungen und Präferenzen: der Einfluss, den Beschränkungen in der Instanziierbarkeit der Strukturelemente einer Konstruktion sowie die Wahl eines lexikalischen Frames in einem Konstrukt auf Koerzionen durch den 
Konstruktions-Frame im Konstrukt-Frame oder die Produktivität der Konstruktion haben (vgl. auch Ziem 2018a: 10);

4. Frame-Nähe: die Frame-zu-Frame-Relationen der in den Konstrukten der Konstruktion evozierten lexikalischen Frames zum Konstruktions-Frame und die Auswirkungen dieser Relationen auf die semantischen Eigenschaften der Konstrukte;

5. Koerzionspotenzial: die Fähigkeit der Konstruktion, in den Konstrukten evozierte lexikalische Frames durch den Konstruktions-Frame zu koerzieren, sie also im Sinne einer Frame-Anpassung in einem Konstrukt-Frame (Unterabschnitt 4.4.1) zu anzupassen;

6. Produktivität: die Erweiterbarkeit der Konstruktion um Konstrukte, die ,neue lexikalische Frames - solche, die nicht in einer Frame-zu-Frame-Relation zum Konstruktions-Frame stehen - evozieren;

7. emergente Struktur: semantische Aspekte, die in einem Konstrukt entstehen, obwohl sie nicht direkt auf eine lexikalische Bedeutung oder die Konstruktionsbedeutung zurückzuführen, im engeren Sinne also nicht kompositionell sind.

Diese Parameter beziehen sich einerseits auf allgemeine (teils formale) Eigenschaften einer Konstruktion, zu denen formale Abstraktheit, Polysemie, Beschränkungen und Präferenzen sowie Produktivität zählen. Andererseits spiegeln sie die Zusammenhänge von lexikalischen Frames und lexikalischen Bedeutungen zu Konstruktions-Frame und Konstruktionsbedeutung wider, was etwa die Parameter Beschränkungen und Präferenzen, Frame-Nähe, Koerzionspotenzial und emergente Struktur betrifft. Hinzu kommt, dass einige der Parameter nicht unabhängig voneinander operieren, sondern in Beziehungen zueinander stehen oder sich gegenseitig bedingen. Diese Verhältnisse sind in Abbildung 5.1 dargestellt.

Die Hierarchie der Parameter, wie sie in Abbildung 5.1 wiedergegeben ist, soll die potenzielle konstruktikographische Relevanz der einzelnen Parameter sowie ihre Beziehungen zueinander verdeutlichen. So stehen formale Abstraktheit der Konstruktion und konstruktionelle Polysemie an der Spitze, da sie den grundsätzlichen konstruktikographischen Umgang mit einer Konstruktion hinsichtlich ihrer semantischen Eigenschaften festlegen, im Falle konstruktioneller Polysemie etwa die Frage danach, ob für eine Konstruktion ein einzelner Konstruktionseintrag angelegt werden muss oder die Polysemie Anlass zu mehreren Konstruktionseinträgen gibt. Die nächsten vier Parameter adressieren konkreter die Verhältnisse zwischen den drei Frame- und Bedeutungstypen (Kapitel 4) oder thematisieren daran anschließende, spezifischere Aspekte. Beschränkungen und Präferenzen sind den Parametern der Frame-Nähe, des Koerzionspotenzials und der Produkti- 


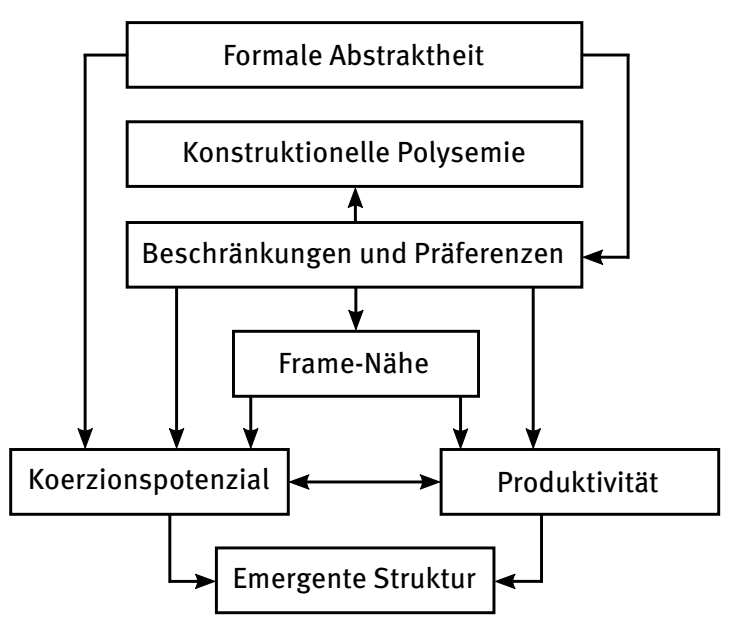

Abb. 5.1: Semantische Parameter von Konstruktionen

vität übergeordnet, da insbesondere Präferenzen für lexikalische Frames sowohl für den Parameter der Frame-Nähe als auch für das Koerzionspotenzial und die Produktivität einer Konstruktion eine Rolle spielen. Auch determinieren lexikalische Frames und lexikalische Bedeutungen zu einem großen Anteil die Polysemie einer Konstruktion. Gleichzeitig werden Beschränkungen und Präferenzen vom Grad der formalen Abstraktheit einer Konstruktion bestimmt. Die Frame-Nähe wiederum ist ein Parameter, auf den Koerzionspotenzial und Produktivität aufbauen. Das Koerzionspotenzial hängt mit der formalen Abstraktheit der Konstruktion zusammen. Koerzionspotenzial und Produktivität stehen darüber hinaus in einer direkten Beziehung zueinander. Von diesen beiden determiniert wird die emergente Struktur, die semantische Leistungen der Konstruktion erfasst, die sich in Standardwerten in Konstruktbedeutungen äußern. Auf diese vielfältigen Beziehungen zwischen den sieben Parametern gehe ich in den einzelnen Abschnitten dieses Kapitels näher ein.

Vorwiegend am Beispiel der reflexiven Bewegungskonstruktion möchte ich die einzelnen Parameter in den folgenden sieben Abschnitten 5.1 bis 5.7 in der eben aufgelisteten Reihenfolge erläutern. Ich gehe dazu vorrangig auf theoretische und ansatzweise auch methodologische Aspekte ein. Wie die semantischen Parameter aus methodischer Sicht ,gemessen“ werden und Eingang in einen Konstruktionseintrag finden können, ist Gegenstand von Kapitel 7. Dort präsentiere ich ebenso die einschlägigen empirischen Ergebnisse zu allen drei untersuchten Konstruktionen, die letztendlich in die drei Konstruktionseinträge im Zusatzmaterial eingehen. 


\subsection{Formale Abstraktheit}

Es ist ein Gemeinplatz der konstruktionsgrammatischen Forschung, ,dass komplexe sprachliche Strukturen selbst bedeutungstragende sprachliche Elemente sind (oder sein können), die sich von Wörtern nur durch ihre Komplexität und/oder Abstraktheit grundsätzlich unterscheiden.“ (Stefanowitsch 2009: 566567). Eine Frage, die sich dabei aufdrängt, ist, ob mit einer hohen formalen Abstraktheit der Konstruktion auch ein hoher Abstraktionsgrad ihrer semantischen Eigenschaften - allen voran: des Konstruktions-Frames - einhergeht, wie es bisweilen angenommen wird (vgl. auch Ziem \& Lasch 2013: 93-94; Szcześniak 2019a: 78): ${ }^{2}$

An einem Ende des Konstruktionskontinuums befinden sich abstrakt-schematische Konstruktionen, die sehr abstrakte Bedeutungen haben (wie die Subjekt-Prädikats-Konstruktion), und am anderen Ende des Kontinuums befinden sich Wörter und Morpheme, die sehr spezielle Bedeutungen aufweisen, die traditionell im Lexikon erfasst werden. Dazwischen befinden sich partiell gefüllte Konstruktionen, die unterschiedlich spezifizierte Bedeutungen haben, wie die Doppelobjektkonstruktion oder die Resultativkonstruktion. (Boas 2019: $246)^{3}$

Bereits in Abschnitt 4.3 habe ich argumentiert, dass sich Konstruktions-Frames nicht grundsätzlich von lexikalischen Frames unterscheiden, was daran sichtbar wird, dass beide identisch sein können. Dies wiederum ist daran zu erkennen, dass bestimmte KtE des KE EREIGNIS der reflexiven Bewegungskonstruktion oder der reflexiven Partikelverbkonstruktion Motion als lexikalischen Frame evozieren. Die Konstrukte in den Belegen in (1) und (2) für die LE bewegen (move.v) und begeben (go.v) sollen dies illustrieren.

2 Ich verwende den Begriff der formalen Abstraktheit als Oberbegriff insbesondere für den Begriff der Schematizität, da Letztere, wie in Unterabschnitt 5.1.2 zu sehen, nur eine von zwei Dimensionen formaler Abstraktheit betrifft. Anders gehen etwa Boas \& Ziem (2018a: 16) vor, die zwischen Schematizität (lexikalischer Spezifiziertheit) und Abstraktheit (Kategorienzugehörigkeit) unterscheiden. Wie Schmid (2020: 229-230) zeigt, entspricht der Begriff der Schematizität nur einer von zwei Dimensionen von Abstraktheit, nämlich derjenigen der Variabilität, die ich als lexikalische Spezifiziertheit bezeichne und in Unterabschnitt 5.1.2 diskutiere.

3 Allerdings handelt es sich bei der Ditransitivkonstruktion (Goldberg 1995: 141-151), die hier als Doppelobjektkonstruktion bezeichnet wird, nicht um eine teilschematische Konstruktion (vgl. auch Boas \& Ziem 2018b: 215-216), zumindest solange man sie nicht, wie etwa Croft (2003: 58), verbspezifisch definiert. 
(1) a. Langsam [Motion bewegte] sich das Skelett durch das Gras. (Glavinic, Thomas: Die Arbeit der Nacht, München Wien: Carl Hanser Verlag 2006, S. 134)

b. Dr. Hans Wilhelm Stein, Burgherr von Saaleck, verbarg die beiden und [Motion begab] sich nach München, in Ehrhardts Hauptquartier, um falsche Pässe und einen Fluchtwagen zu organisieren. (Die Zeit, 30.03.2000, Nr. 14)

(2) Die Schlange [Motion bewegte] sich ein paar Schritte vorwärts auf die Cafetür zu. (Boie, Kirsten: Skogland, Ort: Hamburg 2005, S. 28)

Beachtet man die Tatsache, dass es sich bei LE wie bewegen oder begeben, die Motion evozieren, um lexikalische Konstruktionen handelt (vgl. dazu Ziem 2015d: 56; Ruppenhofer, Boas \& Baker 2018: 489), drängt sich die Frage auf, in welcher Weise sich der Unterschied in der formalen Abstraktheit zwischen ihnen und einer syntaktischen Konstruktion wie der reflexiven Bewegungskonstruktion oder der reflexiven Partikelverbkonstruktion tatsächlich auf die semantischen Eigenschaften beider Arten von Konstruktionen (lexikalische und syntaktische) auswirkt. Ein solcher möglicher Zusammenhang zwischen der formalen Abstraktheit der Konstruktion und der Gestalt ihrer semantischen Eigenschaften scheint in der Konstruktionsgrammatik grundsätzlich angenommen zu werden, wenn Stefanowitsch \& Fischer (2007) festhalten,

dass der Bedeutungsbegriff in der Konstruktionsgrammatik in zweifacher Hinsicht sehr weit gefasst ist: erstens bezüglich des möglichen Abstraktionsgrades einer Konstruktion und zweitens bezüglich der traditionell unterschiedenen Arten von Bedeutungen (z.B. semantische vs. pragmatische Bedeutung). (Stefanowitsch \& Fischer 2007: 205)

Die formale Abstraktheit einer Konstruktion scheint demnach bereits implizit als ein entscheidender semantischer Parameter von Konstruktionen betrachtet zu werden. Es verwundert daher nicht, dass sie sogar als Parameter für die Klassifikation von Konstruktionen herangezogen wird. So verwendet Jacobs (2008: 6-8) das Kriterium der Abstraktheit für gleich drei Kategorien, indem er Konstruktionen nach ihrer (syntaktischen) Komplexität, ihrem (lexikalischen) Spezifiziertheitsgrad und ihrem (semantischen) Abstraktionsgrad klassifiziert. Smirnova \& Mortelmans (2010: 138-139) beziehen sogar ausschließlich Kriterien der Abstraktheit, auf formaler und semantischer Ebene, als Kriterien für die Klassifikation von Konstruktionen ein: Größe, Komplexität und Spezifiziertheit.

Der Vergleich zwischen einer lexikalischen Konstruktion wie der LE bewegen und syntaktischen Konstruktionen wie der reflexiven Bewegungskonstruktion oder der reflexiven Partikelverbkonstruktion legt nahe, dass die formale $\mathrm{Ab}$ - 
straktheit einer Konstruktion nur bedingt mit der semantischen Abstraktheit der Konstruktion korreliert und liefert Evidenz dafür, Konstruktionen nicht grundsätzlich von lexikalischen Bedeutungen unterschiedene ,grammatische‘ Bedeutungen zuzuschreiben (Unterabschnitt 4.3.3). Ein vermeintlicher Grundsatz wie „Je syntaktisch komplexer die Ausdrucksseite, desto semantisch abstrakter die Inhaltsseite“ (Ziem \& Lasch 2013: 93-94) muss deshalb einer differenzierteren Betrachtung unterzogen werden. Diese Beobachtungen sind allerdings kein Argument dafür, die formale Abstraktheit einer Konstruktion als semantischen Parameter von Konstruktionen auszuschließen, denn sie entscheidet gleichwohl über andere Aspekte, die in der konstruktionssemantischen Analyse einer Konstruktion berücksichtigt werden müssen.

- Die formale Abstraktheit einer Konstruktion entscheidet darüber, ob der Konstruktion überhaupt ein symbolischer Charakter und damit semantische Eigenschaften zugeschrieben werden können (vgl. Schmid 2020: 231) oder ob die Annahme einer ,bedeutungslosen' Konstruktion im Sinne von Fillmore, Lee-Goldman \& Rhomieux (2012: 325-328) gerechtfertigt ist.

- Die formale Abstraktheit einer Konstruktion entscheidet darüber, welcher Mechanismus für die Evokation des Konstruktions-Frames verantwortlich gemacht werden kann (dazu Unterabschnitt 8.1.3).

- Die formale Abstraktheit einer Konstruktion entscheidet mit darüber, inwieweit ihre semantischen Eigenschaften überhaupt im Rückgriff auf Frames (und spezieller: auf FrameNet) erfassbar sind und welche Methoden zur Ermittlung des Konstruktions-Frames gewählt werden müssen (dazu Unterabschnitt 8.5.2).

Insbesondere die beiden letztgenannten Punkte legen nahe, dass die Betrachtung der formalen Abstraktheit einer Konstruktion für eine Konstruktionssemantik basal ist. Dies ist der vorrangige Grund, sie in einen semantischen Parameter von Konstruktionen zu überführen. Deshalb möchte ich im Folgenden der Frage nachgehen, wie die formale Abstraktheit einer Konstruktion zu bestimmen ist. Taylor (2004: 51) diskutiert hierfür zwei mögliche Dimensionen, die er zuvorderst als Relationen zwischen Konstruktionen innerhalb eines Konstruktikons versteht: (i) das vertikale Verhältnis zwischen schematischen Konstruktionen und ihren lexikalisch spezifischeren Instanzen und (ii) das horizonale Verhältnis zwischen einer syntagmatisch größeren Konstruktion und kleineren Konstruktionen, die in sie eingebettet werden können. ${ }^{4}$ Ganz ähnlich unterscheidet Schmid (2020: 229-

4 Bei Croft (2001: 17) entsprechen diese Dimensionen den Dichotomien schematisch/spezifisch und atomistisch/komplex (zur deutschen Übertragung vgl. Imo 2007: 33). Jacobs (2016: 26) kom- 
230) die zwei Dimensionen Variabilität und Komplexität. Erstere besteht aus einer Skala zwischen festen bzw. spezifizierten Einheiten auf der einen und variablen bzw. schematischen Einheiten auf der anderen Seite, während Letztere sich auf das Kontinuum zwischen einfachen bzw. kleinen Einheiten und komplexen bzw. großen Einheiten bezieht.

Für eine Konstruktionssemantik lässt sich die formale Abstraktheit einer Konstruktion in Anlehnung an Taylors und Schmids Dimensionen wie folgt reformulieren. Die zwei Eigenschaften, die die formale Abstraktheit einer Konstruktion definieren, sind einerseits die Anzahl ihrer Strukturelemente (Taylors horizontale Dimension, Schmids Dimension der Komplexität) und andererseits ihre lexikalische Spezifiziertheit (Taylors vertikale Dimension, Schmids Dimension der Variabilität). ${ }^{5}$ In den folgenden Unterabschnitten 5.1.1 und 5.1.2 gehe ich auf diese beiden Dimensionen in dieser Reihenfolge ein. Um die Anwendung dieses semantischen Parameters zu demonstrieren, wende ich ihn auf die drei untersuchten Konstruktionen, die reflexive Bewegungskonstruktion, die reflexive Partikelverbkonstruktion und die reflexive Weg-Konstruktion, gleichermaßen an und zeige entsprechende Unterschiede zwischen ihnen auf.

\subsubsection{Anzahl der Strukturelemente}

Die Bestimmung der formalen Abstraktheit einer Konstruktion über die Anzahl ihrer Strukturelemente findet sich außer in den Ansätzen von Taylor (2004: 51) und Schmid (2020: 229-230) in zahlreichen Vorschlägen zur Unterscheidung von Konstruktionen hinsichtlich ihrer formalen Abstraktheit wieder. Croft (2001: 17), Jacobs (2008: 6-8) sowie Traugott \& Trousdale (2013: 11) unterscheiden zwischen atomaren Konstruktionen (etwa LE) und komplexen Konstruktionen (et-

\footnotetext{
biniert solche Dichotomien in der Unterscheidung zwischen substanziellen (atomistischen und spezifischen) und schematischen (komplexen) Konstruktionen. Netzwerktheoretisch ließe sich mit Diessel (2019: 12) in Anlehnung an Schmid (2017, 2020: 25-26) von sequenziellen Relationen einerseits (horizontal) und taxonomischen Relationen andererseits (vertikal) sprechen. Implizit unterscheidet auch Ziem (2018a: 9) zwei vergleichbare Dimensionen für die Musterhaftigkeit einer Konstruktion, nämlich die „syntagmatisch fixierte Abfolge von Konstituenten“ und die Frage, ob sie „lexikalisch spezifiziert [...] oder unspezifiziert“ sind.

5 Diese Definition von Abstraktheit ist damit eine deskriptive, die ich aufgrund ihrer Operationalisierbarkeit für konstruktikographische Zwecke bevorzuge. Eine stärker theoretischgebrauchsbasierte Definition vertreten etwa Clausner \& Croft (1997: 255): „Schematicity is the degree to which one unit (schema) generalizes over many specific units (instantiations)." Die deskriptive Definition stellt allerdings weniger eine Alternative als vielmehr eine veränderte Perspektivierung dar.
} 
wa syntaktischen Konstruktionen). Bei Smirnova \& Mortelmans (2010: 138) wie bei Traugott \& Trousdale (2013: 11) firmiert dieses Kriterium unter der Bezeichnung Größe und unterscheidet etwa Morpheme (als kleinste Konstruktionen) von Argumentstruktur-Konstruktionen (den größten - syntaktischen - Konstruktionen). Im Netzwerkmodell von Diessel (2019: 12) firmieren horizontale Relationen zwischen Strukturelementen unter dem Begriff der sequenziellen Relationen.

Das Kriterium der Anzahl der Strukturelemente ist darüber hinaus dem Lexikon-Grammatik-Kontinuum im Sinne von Langacker (1987: 3) inhärent. Entlang dieser Unterscheidung sieht etwa Goldberg (2003: 219, 2006: 5) Konstruktionen mit mehreren Strukturelementen (etwa die Ditransitivkonstruktion oder Passivkonstruktionen) als komplexer an als Konstruktionen mit nur einem einzigen Strukturelement (etwa lexikalische Konstruktionen oder Morpheme).

Über den konstruktikographischen Beschreibungsapparat, der die Bestandteile einer Konstruktion als KE, KEE und KorE auffasst (Unterabschnitt 2.3.1), ist die Anzahl der Strukturelemente einfach zu erfassen. Als Faustregel kann gelten, dass die formale Abstraktheit einer Konstruktion umso größer ist, je weniger Strukturelemente, also KE, KEE und KorE die Konstruktion besitzt. Umgekehrt ist eine Konstruktion umso weniger formal abstrakt, je mehr Strukturelemente sie aufweist. Die Unterschiede in der Spezifiziertheit der Strukturelemente, also der Unterschied zwischen einem (potenziell) frei besetzbaren KE und einem (mehr oder weniger) lexikalisch spezifizierten KEE oder KorE spielen an dieser Stelle noch keine Rolle.

Die Anzahl der Strukturelemente einer Konstruktion lässt sich nun an die Frage, ob der Konstruktion überhaupt semantische Eigenschaften zukommen, anbinden. Während der reflexiven Bewegungskonstruktion, die über vier Strukturelemente (BEWEGENDES, EREIGNIS, KEE und WEG) verfügt, ebenso wie der reflexiven Partikelverbkonstruktion und der reflexiven Weg-Konstruktion mit jeweils fünf Strukturelementen, Motion als Konstruktions-Frame zugeschrieben werden kann, der zugleich auch als lexikalischer Frame in Erscheinung treten kann, erscheint es nachvollziehbar, dass (syntaktische) Konstruktionen, die hinsichtlich der Anzahl ihrer Strukturelemente weniger komplex sind, bisweilen als ,bedeutungslos' aufgefasst werden. Das typische Beispiel hierfür ist die Subjekt-PrädikatKonstruktion, die - ihrem Namen nach aus nur zwei Strukturelementen bestehend - meist als ,bedeutungslos' analysiert wird (vgl. z.B. Fillmore, Lee-Goldman \& Rhomieux 2012: 326-328; Sag, Boas \& Kay 2012: 15; Boas 2014: 49). Interessanterweise bestehen viele der Konstruktionen, die Fillmore, Lee-Goldman \& Rhomieux (2012: 326-328) als ,bedeutungslos“ klassifizieren, wie die Subjekt-PrädikatKonstruktion aus lediglich zwei Strukturelementen, die sich ebenso bereits an deren Namen ablesen lassen: so etwa die Modifier-Head-Konstruktion oder die Subjekt-Auxiliar-Inversion (dort als Aux-initial bezeichnet). 
Die drei in dieser Arbeit untersuchten Konstruktionen lassen sich hinsichtlich der Anzahl ihrer Strukturelemente am tendenziell konkreten und wenig abstrakten Ende einer formalen Abstraktheitsskala ansiedeln, jedoch untereinander noch einmal hierarchisieren. So verfügt die reflexive Bewegungskonstruktion, wie in einem voll annotierten Konstrukt wie in (3) zu sehen, über vier Strukturelemente: BEWEGENDES, EREIGNIS, KEE und WEG.

(3) Mit jedem Tag deutlicher \{[EReIgnis schält] [KEE sich] [Bewegendes ein unbekanntes Wesen] [WEG aus der groben Form]\}. (Riedel, Susanne: Eine Frau aus Amerika, Berlin: Berlin Verlag 2003, S. 11)

Die reflexive Partikelverbkonstruktion verfügt über fünf Strukturelemente. Für diese Zählung ist es unerheblich, ob das Nicht-Kern-KE 〈WEG〉, wie in (4), uninstanziiert bleibt oder ob es, wie in (5), instanziiert wird. Die Anzahl der Strukturelemente liegt gegenüber derjenigen der reflexiven Bewegungskonstruktion höher, da die reflexive Partikelverbkonstruktion über ein zweites KEE, nämlich RICHTUNG, verfügt. Dies gilt auch dann, wenn die Partikel, wie in (6), in Kontaktstellung zum KtE von EREIGNIS instanziiert wird.

(4) Ein Buch aus lauter tiefgefrorenen Kapiteln - Tödlicher Frost, \{[BEwEgendes man] [EReignis friert] [KEE sich] [RIchtung durch]\}. (Die Zeit, 05.01.2000, Nr. 2)

(5) Ein Motor dröhnt von der Straße herüber, und \{[BEwEGENDES diese dröhnende Straße] [EREIGnis fräst] [KEE sich] [〈WEG in mein Gedächtnis] [RIchtung ein]\}. (Riedel, Susanne: Eine Frau aus Amerika, Berlin: Berlin Verlag 2003, S. 95)

(6) Ein geschickter Deal, mit dem $\left\{\left[_{\mathrm{KeE}} \mathrm{sich}\right]_{\text {[Bewegendes }} \mathrm{AOL}\right]_{\langle\mathrm{WEg}\rangle}$ in die Multimedia-Distribution] [RIchtung ein][EReignis kaufte]\}. (Die Zeit, 23.03.2000, Nr. 13)

Ebenso wie die reflexive Partikelverbkonstruktion verfügt auch die reflexive WegKonstruktion über fünf Strukturelemente, da sie gegenüber der reflexiven Bewegungskonstruktion ein zusätzliches KorE besitzt. Zum Vergleich sei in (7) noch einmal ein voll annotiertes Konstrukt dargestellt.

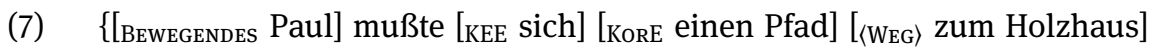
[EReIgnis bahnen]\}. (Koneffke, Jan: Paul Schatz im Uhrenkasten, Köln: DuMont Buchverlag 2000, S. 77)

Die reflexive Partikelverbkonstruktion und die reflexive Weg-Konstruktion sind lexikalisch spezifizierter als die reflexive Bewegungskonstruktion, da sie jeweils nicht nur über ein, sondern gleich über zwei lexikalisch spezifizierte Elemente verfügen. Die reflexive Weg-Konstruktion ist wiederum spezifischer als die reflexive 
Partikelverbkonstruktion, da ihr KorE lexikalisch nahezu vollständig invariant ist (neben dem Nomen Weg sind nur die beiden semantisch verwandten Nomen Pfad und Trampelpfad belegt). Das KorE unterscheidet sich darin von dem als Reflexivum instanziierten KEE der reflexiven Partikelverbkonstruktion ebenso wie von dem durch eine größere Bandbreite an lexikalischem Material instanziierbaren KEE RICHTUNG. Damit ist klar, dass die Anzahl der Strukturelemente nicht allein ausschlaggebend für die Bestimmung der formalen Abstraktheit einer Konstruktion sein kann. Besonders deutlich wird dies, wenn man die Untersuchung syntaktischer Konstruktionen verlässt und zu lexikalischen Konstruktionen übergeht (vgl. ähnlich Ziem \& Lasch 2013: 94, Anm. 54). Die Klasse der lexikalischen Konstruktionen, also LE, konstituiert sich überwiegend aus Konstruktionen, die aus lediglich einem Strukturelement bestehen (z.B. einem monomorphematischen Wort) ${ }^{6}$ Somit ist klar, dass neben der Anzahl der Strukturelemente, die allein noch kein verlässlicher Maßstab ist, auch die lexikalische Spezifiziertheit einer Konstruktion in die Bestimmung ihrer formalen Abstraktheit eingehen muss.

\subsubsection{Lexikalische Spezifiziertheit}

Die lexikalische Spezifiertheit einer Konstruktion kann als noch wichtigerer Maßstab für ihre formale Abstraktheit als die Anzahl ihrer Strukturelemente gelten. Für die Frage nach den semantischen Eigenschaften einer Konstruktion ist dieses Kriterium wesentlich, weil es über den Mechanismus zur Evokation des Konstruktions-Frames entscheidet (vgl. Unterabschnitt 8.1.3) und darüber Auskunft gibt, inwieweit sich eine komplexe syntaktische Konstruktion von einer (weniger komplexen) lexikalischen Konstruktion, also einer LE, unterscheidet. Lexikalische Spezifiziertheit ist auch in der Literatur noch vor der Anzahl der Strukturelemente ein verbreiteterer Maßstab für die formale Abstraktheit von Konstruktionen und geht nicht erst auf Taylor (2004: 51) oder Schmid (2020: 229230) zurück. So beruht das Konzept der Mini-Konstruktionen von Boas (z.B. 2003a, 2011b) auf diesem Kriterium, da Mini-Konstruktionen stets verbspezifisch, also auf eine LE als elementaren Bestandteil der Konstruktion ausgerichtet sind. Auch Iwata (2008: 35-38), der zwischen die Abstraktheitsgrade einer Konstruktion und ihrer Konstrukte noch die hierarchischen Ebenen der verbklassen- und verbspezifischen Konstruktionen einzieht (vgl. dazu schon Croft 2003: 58), verwendet die lexikalische Spezifiziertheit von Konstruktionen durch Verben als Grundlage

\footnotetext{
6 Vgl. hierzu Diessel (2019: 11), der auf Basis von Langacker (1987: 82) als Kriterium für eine syntaktische Konstruktion ihre Konstitution aus mindestens zwei ,bedeutungstragenden` Elementen festhält.
} 
für die Unterscheidung ihrer formalen Abstraktheit. Implizit liegt das Kriterium der lexikalischen Spezifiziertheit auch der Unterscheidung in Makro-, Meso- und Mikro-Konstruktionen und -Konstrukte von Traugott (2008b: 31-32, 2008a: 8) zugrunde. Smirnova \& Mortelmans (2010: 139) sprechen in semantischer Hinsicht von der Spezifität einer Konstruktion und nennen lexikalische Konstruktionen (LE) als spezifischer als etwa Derivationsmorpheme. Der Abstraktheitsbegriff von Engelberg (2019: 16) beruht gar allein auf lexikalischer Spezifiziertheit. Diessel (2019: 12) stellt solche taxonomischen (vertikalen) Relationen komplementär sequenziellen (horizontalen) Relationen zur Seite.

Die lexikalische Spezifiziertheit ist als Maßstab für den Parameter der formalen Abstraktheit einer Konstruktion so entscheidend, weil von ihr vermutet wird, dass sie unmittelbaren Einfluss auf die ,Bedeutungshaltigkeit‘ einer Konstruktion hat. Konstruktionen, die lexikalisch (teil-)spezifiziert sind, gelten aufgrund dieser Eigenschaft als ,bedeutungshaltiger‘ als Konstruktionen ohne lexikalische Spezifizierung (vgl. auch Szcześniak 2019a: 77):

Exactly how contentful a construction's meaning is should correlate with how lexically filled it is, and the more substantive meanings should be accounted for by reference to the lexical item inclusions present in the construction. (Szcześniak 2013: 190-191)

Diesem Grundsatz liegt freilich die Annahme zugrunde, dass lexikalisch weniger spezifizierten Konstruktionen stets weniger semantische Eigenschaften zukommen als solchen, die lexikalisch spezifiziert sind:

\footnotetext{
Just how rich a meaning of a construction is, depends on the degree to which it is substantive. While completely abstract forms are spare in meaning, the closer an item is to the lexical end of the continuum (that is, the more it is filled with lexical material) the richer its meaning. (Szcześniak 2014b: 19)
}

Die lexikalische Spezifiziertheit einer Konstruktion müsste dann also idealerweise proportional mit deren ,Bedeutungshaltigkeit‘ korrelieren:

\footnotetext{
It is through the fixed lexical material that the meaning of a construction can be enriched. I do not take issue with the idea that grammatical constructions are characterized by varying degrees of semantic content, but as a rule of thumb, the more lexically specified a construction, the more contentful the meaning it can carry. (Szcześniak 2014b: 28)
}

Was dieser Vorstellung von der ,Bedeutungshaltigkeit‘ allerdings fehlt, ist ein Konzept, wie ebendiese zu bestimmen ist. Was also bedeutet es, dass eine Konstruktion ,bedeutungshaltiger' ist als eine andere? Bevor ich eine Antwort auf diese Frage diskutiere, möchte ich zunächst definieren, wie sich die lexikalische Spezifiziertheit einer Konstruktion mit Hilfe des konstruktikographischen Beschrei- 
bungsapparates erfassen lässt. Grundsätzlich lässt sich die lexikalische Spezifiziertheit einer Konstruktion über das Verhältnis von KE, KEE und KorE bestimmen. Eine Konstruktion, die ausschließlich aus KE als ,constituents, or slots, of a grammatical construction“ (Ziem \& Flick 2019: 204; Ziem, Flick \& Sandkühler 2019: 68) besteht, ist lexikalisch unspezifiziert. Eine Konstruktion, die aus einem (oder mehreren) KEE als „lexically specified CE“ (Ziem \& Flick 2019: 204; Ziem, Flick \& Sandkühler 2019: 68) oder KorE als „word, or a string of words“ (Ziem \& Flick 2019: 205; Ziem, Flick \& Sandkühler 2019: 69) in Verbindung mit einem oder mehreren KE besteht, ist lexikalisch teilspezifiziert. Eine Konstruktion, die ausschließlich aus LE (bzw. KEE oder KorE) besteht und keine KE aufweist, ist lexikalisch voll spezifiziert. ${ }^{7}$ Boas \& Ziem (2018b) weisen in diesem Sinne darauf hin, dass eine Konstruktion umso seltener ein KEE enthält, je abstrakter sie ist:

The more schematic a construction gets, that is, the more a construction is located towards the grammar pole in the lexicon-grammar continuum, the more likely it is that it does not include one or more fixed lexical items. The ditransitive [sic!] construction, for example, is defined by its structural properties alone. (Boas \& Ziem 2018b: 215-216)

Boas (2017) geht demgegenüber noch weiter und setzt das Vorhandensein oder Nicht-Vorhandensein eines KEE direkt mit der ,Bedeutungshaltigkeit‘ gleich:

Non-lexical constructions without meaning (or without [sic!] very little clearly identifiable meaning) such as Subject_predicate, Gapping, and Right_Node_Raising are not evoked by a CEE. Lexical constructions, (semi-)idiomatic constructions, argument structure constructions, and other meaningful constructions will list a specific CEE. (Boas 2017: 568-569)

Fest steht zudem, dass die lexikalische Spezifiziertheit im Zusammenhang mit der Anzahl der Strukturelemente einer Konstruktion betrachtet werden muss: Eine große Anzahl von Strukturelementen kann zugleich eine voll spezifizierte Konstruktion ergeben, während eine geringe Anzahl von Strukturelementen eine unspezifizierte Konstruktion ergeben kann. ${ }^{8}$ Ein Beispiel für ersteren Fall wäre ein substanzielles Idiom im Sinne von Fillmore, Kay \& O’Connor (1988:

7 Bei einer lexikalisch voll spezifizierten Konstruktion würde man ihre Strukturelemente wohl eher als KE denn als KEE oder KorE bezeichnen, obwohl sie der eigentlichen Definition von KE als (mehr oder weniger) produktiv instanziierbaren Strukturelementen damit zuwiderlaufen. Um Missverständnisse zu vermeiden, bezeichne ich diese als LE, um deutlich zu machen, dass sie keine syntaktischen Konstruktionen im engeren Sinne sind, was bereits daran zu erkennen ist, dass sie so stark lexikalisch fixiert sind, dass sie selbstständig einen Frame evozieren und damit wie alle anderen LE im FrameNet behandelt werden können.

8 Auch Diessel (2019: 64) weist darauf hin, dass sequenzielle (d.h. horizontale) und taxonomische (d.h. vertikale) Relationen in einem engen Zusammenhang zueinander stehen. 
505-506), eines für letzteren Fall die Subjekt-Prädikat-Konstruktion, wie Fillmore, Lee-Goldman \& Rhomieux (2012: 326) sie annehmen. Allerdings weist Taylor (2004: 51) darauf hin, dass beide Dimensionen grundsätzlich unabhängig voneinander sind: So kann eine voll lexikalisch spezifizierte Konstruktion ebenso wie eine in dieser Hinsicht vollkommen schematische Konstruktion horizontal komplex sein, also eine große Anzahl von Strukturelementen aufweisen. Gleichzeitig können lexikalisch stark spezifizierte Konstruktionen horizontal ebenso simpel wie komplex sein. Ersteres gilt für lexikalische Konstruktionen, Letzteres erneut für substanzielle Idiome oder auch idiomatische Sätze im Sinne von Finkbeiner (2008).

Die drei untersuchten Konstruktionen lassen sich nun wie folgt hinsichtlich ihrer lexikalischen Spezifiziertheit bestimmen. Die reflexive Bewegungskonstruktion, die über insgesamt vier Strukturelemente verfügt, schließt eines davon als KEE ein. Sie ist damit teilspezifiziert. Das Verhältnis zwischen KE und KEE beträgt bei ihr 3:1. Die reflexive Partikelverbkonstruktion, die über fünf Strukturelemente verfügt, kommt dadurch, dass sie zwei KEE (das als Reflexivum instanziierte KEE sowie das KEE RICHTUNG) aufweist, auf ein anderes Verhältnis. Mit beachtet werden muss auch ihr Nicht-Kern-KE $\langle\mathrm{WEG}\rangle$, weshalb sie dann mit instanziiertem $\mathrm{KE}$ 〈WEG $\rangle$ auf ein Verhältnis von 3:2 kommt, ohne instanziiertes KE 〈WEG〉 auf 2:2. Die reflexive Weg-Konstruktion, die ebenfalls über fünf Strukturelemente verfügt und eines davon als KEE sowie eines als KorE besitzt, kommt auf ein Verhältnis zwischen KE und KEE/KorE von 3:2. Da auch sie über ein Nicht-Kern-KE verfügt, beträgt das Verhältnis ohne instanziiertes KE $\langle\mathrm{WEG}\rangle$ wie bei der reflexiven Partikelverbkonstruktion 2:2.

Auf einer Skala lexikalischer Spezifiziertheit lassen sich die Konstruktionen wie folgt verorten: Die reflexive Weg-Konstruktion ist am spezifischsten. ${ }^{9}$ Danach folgt die reflexive Partikelverbkonstruktion, da ihre beiden KEE, wie in Unterabschnitt 5.1.1 argumentiert, weniger lexikalisch fest sind als das KEE und insbesondere das KorE der reflexiven Weg-Konstruktion. Noch weniger spezifisch ist schließlich die reflexive Bewegungskonstruktion, da ihr gegenüber der reflexiven Partikelverbkonstruktion ein KEE und gegenüber der reflexiven Weg-Konstruktion das KorE fehlt. In Abbildung 5.2 ist das Verhältnis der drei Konstruktionen nach dem Grad ihrer formalen Abstraktheit dargestellt.

9 Gestützt wird dieses Ergebnis durch die Beobachtung von Broccias (2012: 741), der für die strukturell äquivalente way-Konstruktion festhält, dass sie „,should probably be considered to be on the lexical side of the [syntax-lexicon, A.W.] continuum“, wobei dies - wie im Vergleich der drei hier untersuchten Konstruktionen - mit Sicherheit nur relativ zu beantworten ist. Vgl. kritisch zu Broccias’ Einschätzung Traugott \& Trousdale (2013: 90). 


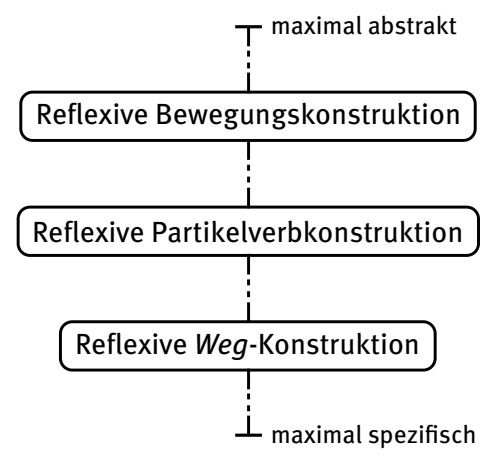

Abb. 5.2: Verhältnis von reflexiver Bewegungskonstruktion, reflexiver Partikelverbkonstruktion und reflexiver Weg-Konstruktion nach dem Grad ihrer formalen Abstraktheit

Da die drei untersuchten Konstruktionen nun hinsichtlich ihrer formalen Abstraktheit bestimmt sind, kann noch einmal die Frage, wie diese formale Abstraktheit der Konstruktionen mit ihrer semantischen Abstraktheit in Zusammenhang steht, in den Blick genommen werden. Anders als es die oben zitierte Argumentation von Szcześniak (2013: 190-191, 2014b: 28, 2019a: 77) erwarten lassen würde, ist diese Skala lexikalischer Spezifiziertheit allein nicht mit der ,Bedeutungshaltigkeit" der Konstruktionen zu korrelieren. So weisen die reflexive WegKonstruktion und die reflexive Bewegungskonstruktion unterschiedliche Grade an formaler Abstraktheit auf, die auf eine spezifischere semantische Leistung der Ersteren hindeuten müssten. Dies allerdings ist nicht der Fall: Beide Konstruktionen besitzen als Konstruktions-Frame Motion. Die lexikalische Spezifiziertheit bestimmt damit nicht grundsätzlich über den Konstruktions-Frame, sondern determiniert vielmehr den Mechanismus, nach dem er evoziert wird. Bevor ich in Unterabschnitt 8.1.3 darauf eingehe, sei an dieser Stelle soviel gesagt: Die reflexive Bewegungskonstruktion kann auch deshalb als abstrakter als etwa die reflexive Weg-Konstruktion gelten, weil ihre lexikalische Spezifizierung nicht für die Evokation des Konstruktions-Frames verantwortlich gemacht werden kann. Mit anderen Worten: Ein Reflexivum wie sich kann kaum als LE, die Motion evoziert, betrachtet werden (dazu Unterabschnitt 8.3.1). Dies gilt indes auch für das KEE RICHTUNG der reflexiven Partikelverbkonstruktion, weshalb diese auf der Abstraktheitsskala zwischen der reflexiven Bewegungskonstruktion und der reflexiven Weg-Konstruktion liegt. Für die reflexive Weg-Konstruktion schließlich beantwortet sich die Frage nach der Evokation des Konstruktions-Frames anders: Das Nomen Weg als Bestandteil des KorE ist viel eher dazu geeignet, Motion zu evozieren (vgl. Unterabschnitt 8.3.2). 
Die formale Abstraktheit einer Konstruktion in Gestalt des Kriteriums der lexikalischen Spezifizierung trifft also eine Aussage über die Relationen zwischen der Form der Konstruktion und ihren semantischen Eigenschaften - konkret: der Fähigkeit der Formseite zur Evokation des Konstruktions-Frames. Eine abstraktere Konstruktion evoziert ihren Konstruktions-Frame in anderer Weise als eine spezifischere Konstruktion. Somit ist auch die Gleichsetzung des Vorhandenseins eines KEE mit der ,Bedeutungshaltigkeit‘ und des Nicht-Vorhandenseins eines KEE mit der ,Bedeutungslosigkeit‘ einer Konstruktion, wie sie Boas (2017: 568-569) vornimmt, unzutreffend: Die Ditransitivkonstruktion etwa, die über kein KEE verfügt, ist als ebenso ,bedeutungshaltig' zu klassifizieren wie die reflexive Bewegungskonstruktion, die über ein eher abstraktes KEE verfügt, die reflexive Partikelverbkonstruktion, die über zwei vergleichbar abstrakte KEE verfügt, und die reflexive Weg-Konstruktion, die neben dem allen drei Konstruktionen gemeinsamen KEE über ein konkreteres KorE verfügt.

Damit ist allerdings ebenso deutlich, dass es nicht allein darauf ankommt, wie stark eine Konstruktion lexikalisch spezifiziert ist, wie sich also das Verhältnis von KE, KEE und KorE zusammensetzt, sondern dass auch die Art der lexikalischen Spezifizierung eine Rolle spielt. So kann eine Konstruktion wie die reflexive Bewegungskonstruktion, die als KEE ein Reflexivum besitzt, allein schon deshalb als abstrakter gelten, weil das Reflexivum nicht allein in der Lage ist, den Konstruktions-Frame zu evozieren. Gleiches gilt für die reflexive Partikelverbkonstruktion, die mit RICHTUNG zwar ein KEE mehr besitzt, dieses aber ebenso nicht in der Lage ist, selbstständig einen Frame wie Motion zu evozieren. Die reflexive Weg-Konstruktion hingegen ist allein deshalb spezifischer, weil ihr KorE dazu sehr wohl in der Lage ist. Die reine Anzahl der Strukturelemente (Unterabschnitt 5.1.1) ist in diesen Fällen lediglich zweitrangig, wie ein Vergleich von Konstrukten der reflexiven Weg-Konstruktion in (8) mit einigen Konstrukten der nichtreflexiven Weg-Konstruktion zeigt, ${ }^{10}$ die ich in (9) wiederhole und analog zu den Strukturelementen der reflexiven Weg-Konstruktion annotiere. ${ }^{11}$

(8) a. Dann $\{$ [EREIGnis bahnte] [Bewegendes ich] [KeE mir] [KorE einen Weg] [〈Weg durch allerlei Buschwerk und trockenes Gehölz]\}. (Düffel, John von: Vom Wasser, München: dtv 2006, S. 214)

b. \{[Bewegendes Mein Schälmesser mit der dünnen Klinge] [EReIgnis säbelt]

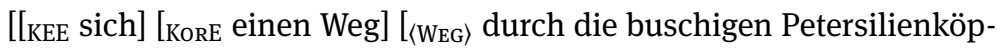
fe]\}, während ich überlege, ob es tatsächlich Köpfe oder doch Blätter

10 Zur Herkunft dieser Daten vgl. Unterabschnitt 3.4.3.

11 Aufgrund der Koordinationsellipsen in den Konstrukten in (9) ist das KE BEWEGENDES dort jeweils nicht instanziiert und deshalb auch nicht annotiert. 
oder gar Büschel heißt. (Riedel, Susanne: Eine Frau aus Amerika, Berlin: Berlin Verlag 2003, S. 106)

c. $\{[$ Bewegendes Paul] mußte [KeE sich] [Kore einen Pfad] [〈WeG $\rangle$ zum Holzhaus] [EREIGnis bahnen]\}. (Koneffke, Jan: Paul Schatz im Uhrenkasten, Köln: DuMont Buchverlag 2000, S. 77)

(9) a. Fünf Minuten knapp sprach sie, dann nickte Pardell mehrmals und $\left\{\left[\right.\right.$ EREIGNis bahnte] [KorE einen Weg] [ ${ }_{\langle\mathrm{WEG}\rangle}$ durch die Bevölkerung des Gran Tour]\}, die Frau folgte ihm. (Kopetzky, Steffen: Grand Tour, Frankfurt am Main: Eichborn 2002, S. 504)

b. Wer sich dennoch einen Menschen vorstellen möchte und $\{[$ KorE seinen eigenen Weg] [〈WEG durch die Kapitel] [EREIGNIS bahnt]\}, kommt mit dieser gewaltigen Bestandsaufnahme weiter als je zuvor. (Die Zeit, 23.03.2000, Nr. 13)

c. Ich nahm seine Fährte auf und \{[EReIGnis bahnte] [KorE meinen Weg] [ ${ }_{\text {WEG }}$ durch den Sand $]$, vorbei an den flanierenden Pärchen und wasserscheuen Zauderern, die bis zu den Knien auf die Wellen zustaksten, um dann wieder das Trockene zu suchen. (Düffel, John von: Vom Wasser, München: dtv 2006, S. 245)

Die nicht-reflexive Weg-Konstruktion verfügt, da das Reflexivum wegfällt, über dieselbe Anzahl an Strukturelementen wie die reflexive Bewegungskonstruktion (sofern man das vermutlich als Nicht-Kern-KE einzustufende KE 〈WEG〉 der nicht-reflexiven Weg-Konstruktion mitzählt) und auch das Verhältnis von KE und $\mathrm{KEE} /$ KorE ist mit 3:1 identisch. Der einzige Unterschied zur reflexiven Bewegungskonstruktion liegt in der Art des lexikalisch spezifizierten Elements, hier also des KorE: Der Kopf der entsprechenden NP, das Nomen Weg (oder ein semantisch verwandtes Nomen), kann vermittels eines Spreading-Activation-Prozesses als Motion evozierend angesehen werden (dazu Unterabschnitt 8.3.2). Aus diesem Grund ist die nicht-reflexive Weg-Konstruktion als weniger abstrakt als die reflexive Bewegungskonstruktion einzustufen, trotz derselben Anzahl der Strukturelemente und desselben Verhältnisses von KE und KEE/KorE.

Die Frage nach der lexikalischen Spezifiziertheit einer Konstruktion muss demnach um die Frage nach der Art dieser lexikalischen Spezifiziertheit erweitert werden. Dies gilt nicht nur für Argumentstruktur-Konstruktionen, sondern insbesondere für weniger komplexe, lexikalische Konstruktionen und insbesondere Morpheme. Denn wie neben Taylor (2004: 51) auch Smirnova \& Mortelmans (2010: 139-140) festhalten, sind die Anzahl der Strukturelemente (Größe der Konstruktion) und ihre lexikalische Spezifiziertheit nicht miteinander korrelierbar: So weisen zwar Derivationsmorpheme die geringste Anzahl an Strukturelementen auf und sind vollständig lexikalisch spezifiziert, allerdings sind sie semantisch 
stark abstrakt. Boas \& Ziem (2018a: 16) begründen diesen Aspekt (den sie unter den Begriff der Abstraktheit fassen) über die Kategorienzugehörigkeit des betreffenden Elements: Einzelne LE sind demzufolge konkreter als die Kategorien (z.B. die Wortarten), denen sie zugeordnet werden können.

Die angesprochene Kategorienzugehörigkeit lässt sich freilich nicht nur auf den Unterschied zwischen Kategorie (hier: Nomen) und Instanz (etwa: Weg in der reflexiven Weg-Konstruktion) beziehen, sondern ebenso über Kategorien hinweg. So erscheint es plausibel, dass eine Kategorie ,Nomen' tendenziell eher dazu in der Lage ist, die Spezifiziertheit der Konstruktion zu erhöhen als eine Kategorie wie derjenigen der Reflexiva oder Verbpartikeln. Durch diese Kategorienzugehörigkeit der Strukturelemente einer Konstruktion entscheidet sich darüber hinaus die eingangs angesprochene Frage nach der Fähigkeit, Konstruktions-Frames überhaupt annehmen zu können. Während dies für Nomen wie Weg, die bereits genuin als LE von Frames infrage kommen, unproblematisch erscheint (Unterabschnitt 8.3.2), steht der Status etwa eines Reflexivums oder einer Partikel als frame-evozierende LE durchaus infrage. Was diese beiden Elemente aber in jedem Fall unterscheidet, ist, dass das Nomen Weg (ebenso wie semantisch verwandte Nomen) in der Lage ist, zumindest indirekt Motion zu evozieren, während ein Reflexivum oder eine Partikel, wenn sie überhaupt als frame-evozierend betrachtet werden, sicher einem anderen Frame zugeordnet werden müssten (vgl. Unterabschnitt 8.3.1 für eine ausführlichere Analyse).

Die Tragweite dieses Aspekts ist freilich nicht allein über eine homogene Klasse von Argumentstruktur-Konstruktionen - und erst recht nicht nur mit den drei hier untersuchten, eng verwandten Konstruktionen - zu beantworten, vielmehr müssen weitere Studien auch anderer Konstruktionen zeigen, wie weit ein framesemantischer Ansatz einer Konstruktionssemantik gehen kann (vgl. auch Hilpert 2019: 57):

Da Goldbergs (1995) Analyse von Argumentstrukturkonstruktionen in der Entwicklung und der Rezeption der Konstruktionsgrammatik einen unverhältnismäßig großen Raum einnimmt, entsteht manchmal der Eindruck, dass die Konstruktionsgrammatik für alle sprachlichen Regularitäten eine feste Verknüpfung mit spezifischen framesemantischen Inhalten annimmt. Das ist aber ein grundsätzliches Missverständnis: Bedeutungen können in der Konstruktionsgrammatik wesentlich abstrakter sein und sich auch auf Bedeutungsebenen beziehen, die mit Framesemantik nichts zu tun haben. (Stefanowitsch 2011: 17-18)

Die Grenzen der Leistungsfähigkeit der Frame-Semantik sind allerdings, anders als es Stefanowitschs pauschale Einschätzung suggeriert, keineswegs empirisch bereits abgesteckt. Die formale Abstraktheit einer Konstruktion ist einer der Parameter, die bei einer Annäherung an eine Aussage über diese Leistungsfähigkeit berücksichtigt werden müssen. Bis dahin ist die weiter oben aufgeworfene Fra- 
ge nach der ,Bedeutungshaltigkeit‘ einer Konstruktion weniger eine grundsätzlich frame-semantisch zu problematisierende, sondern sie lässt sich reformulieren als Frage nach dem Mechanismus, über den eine Konstruktion aufgrund ihrer formalen Abstraktheit ihren Konstruktions-Frame evoziert. Die Abstraktheit einer Konstruktion ist als semantischer Parameter von Konstruktionen deshalb so elementar, weil sie eine wesentliche Auskunft über die Relationen zwischen der ihrer Formseite und ihrem Konstruktions-Frame (Kapitel 8) Auskunft gibt.

Im Rückblick auf den im Eingang zu diesem Kapitel in Abbildung 5.1 dargestellten Zusammenhang aller semantischen Parameter von Konstruktionen ist festzuhalten, dass die formale Abstraktheit einer Konstruktion insbesondere mit ihren Beschränkungen und Präferenzen (Abschnitt 5.3.1) sowie mit ihrem Koerzionspotenzial (Abschnitt 5.5) in Verbindung steht. Ersteres ist offensichtlich, denn je weniger lexikalisch spezifiziert eine Konstruktion ist, desto weniger Beschränkungen ihrer einzelnen Strukturelemente sind zu erwarten. Das Koerzionspotenzial bestimmt sich indes durch Koerzionseffekte, die wesentlich von der Instanziierung einzelner Strukturelemente, im Falle der drei untersuchten Konstruktionen insbesondere des KE EREIGNIS, abhängen.

\subsection{Konstruktionelle Polysemie}

Ebenso wie lexikalische Konstruktionen (also LE im Sinne von FrameNet) können auch syntaktische Konstruktionen polysem sein (vgl. z.B. Taylor 2012: 219). ${ }^{12}$ Polysemie von Konstruktionen muss ebenso frame-semantisch erfassbar sein, wie dies für LE der Fall ist, wobei die Frage danach, wie dies für syntaktische Konstruktionen geschehen kann, noch weitgehend unbeantwortet ist. Als Annäherung an dieses Problem erscheint deshalb zunächst ein Blick auf den Umgang mit der Polysemie lexikalischer Konstruktionen angebracht. Wie in Unterabschnitt 2.1.2 dargestellt, behandelt FrameNet polyseme Wörter als mehrere, voneinander unterschiedene LE. ${ }^{13}$ Um ein einfaches Beispiel zu nennen: Für das Verb erscheinen

12 Vor- und Nachteile der Annahme von Polysemie aus konstruktionsgrammatischer Sicht im Vergleich zu einem Monosemie-Ansatz diskutiert Boogaart (2009: 217-228). In jedem Fall ist die Kritik von Schmid (2020: 27), Konstruktionen implizierten ,the existence of fairly neat one-to-one correspondences between forms and meanings" keineswegs zutreffend, wenn man konstruktionelle Polysemie erlaubt.

13 Dies gilt nach der Einschätzung von Busse (2012: 652) für die Frame-Semantik generell: „Eine Frame-semantische Analyse zielt immer auf Lesarten (Teilbedeutungen), nicht auf Lemmata (lexikalische Wörter bzw. Wortformen).“ 
(appear.v) finden sich im DWDS-Kernkorpus 21 mindestens die vier in den Belegen (10)-(13) zu erkennenden Lesarten. ${ }^{14}$

(10) Inzwischen aber ist eine weitere Figur erschienen, die kaum erkennbar neben dem Sessel steht. (Beyer, Marcel: Spione, Köln: DuMont 2000, S. 305)

(11) Diese sind begeistert, und als Zeichen der Reunion erscheint das Wort „PizzaHut.“ (Schuh, Franz: Schreibkräfte, Köln: DuMont 2000, S. 8)

(12) Zudem erscheint das Bild ein wenig verschwommen, ein weiterer Hinweis auf Staub. (Beyer, Marcel: Spione, Köln: DuMont 2000, S. 35)

(13) Heutzutage erscheinen in erster Linie Bücher, die nicht von Literaten geschrieben worden sind. (Schuh, Franz: Schreibkräfte, Köln: DuMont 2000, S. 54)

Im Lexical Unit Index von FrameNet 1.7 findet sich die LE appear.v dementsprechend gleich mehrmals: Sie kann, wie in (10), den Frame Arriving evozieren, wie in (11) den Frame Becoming_visible, wie in (12) den Frame Give_impression oder wie in (13) den Frame Publi shing. Die Polysemie des Verbs erscheinen kann demnach als Evokation verschiedener Frames durch eine einzige lexikalische Form dargestellt werden. In den Belegen (10)-(13) sind deshalb vier verschiedene LE erscheinen zu sehen, die jeweils einen anderen Frame evozieren.

Das Phänomen der Polysemie auf der Ebene solcher lexikalischer Konstruktionen gilt nun grundsätzlich analog für syntaktische Konstruktionen. Wie bereits Goldberg (1996) bemerkt, besteht zwischen der Polysemie von LE und derjenigen von syntaktischen Konstruktionen kein genereller Unterschied:

In a theory of grammar such as Construction Grammar, which posits no strict division between the lexicon and the rest of grammar, such a parallelism between lexical and constructional polysemy is natural and, indeed, expected. (Goldberg 1996: 51)

Freilich ist die Annahme von konstruktioneller Polysemie und insbesondere die Frage danach, ob Polysemie eher auf der Ebene syntaktischer Konstruktionen oder lexikalischer Bedeutungen anzunehmen ist, alles andere als unstrittig. So argumentiert vor allem Boas (2002: 132-134, 2003a: 98-99, 2010b: 58-60, 2011a: 1275, 2011b: 50-51) in zahlreichen Arbeiten gegen Goldbergs Vorgehensweise und für die präzisere Erfassung von Polysemie auf der Ebene lexikalischer Bedeutungen durch sein Konzept der Mini-Konstruktionen. Für verwandte Ideen plädieren auch Nemoto (1998: 239, 2005: 131-133) und Iwata (2005b: 115-116, 2008: 37). Ich möchte im Folgenden allerdings argumentieren, dass Polysemie über die Frage,

14 Zur Herkunft dieser Daten vgl. Unterabschnitt 3.4.3. 
ob sie auf der Ebene des Konstruktions-Frames oder der lexikalischen Frames (und der aus ihnen resultierenden lexikalischen Bedeutungen) angesetzt werden soll, hinausgeht und zum Teil deutlich subtilere Aspekte der semantischen Eigenschaften einer Konstruktion erfasst.

Ich beginne erneut mit der reflexiven Bewegungskonstruktion. Schon ihr englisches Pendant, die way-Konstruktion, ist mit ihren Lesarten ,manner', ,means“ und ,incidental' sowie einer neutralen Lesart, auf die ich in Unterabschnitt 3.1.3 hingewiesen habe, ein Paradefall für konstruktionelle Polysemie (vgl. auch Goldberg 1996: 51):

The way construction demonstrates the need to recognize constructional polysemy, parallel to the polysemy often posited for lexical items and grammatical morphemes. (Goldberg 1995: 218)

Die Gründe, Polysemie bei Konstruktionen eine ebenso große Rolle wie bei LE einzuräumen und für sie einen eigenen semantischen Parameter anzusetzen, sind auf konstruktikographische Interessen zurückzuführen. Schon bei der Polysemie lexikalischer Konstruktionen stellt sich die Frage, ob deren unterschiedliche Lesarten in einer einzelnen lexikographischen Beschreibungseinheit zusammengefasst werden sollen oder ob sie in unterschiedliche Einheiten getrennt werden sollen. Ersteres wird allgemein als Lumping, Letzteres als Splitting bezeichnet (vgl. z.B. Taylor 2012: 223-228). FrameNet wählt tendenziell den Weg des Splittings: „A frames approach to lexical semantics, [...], is more or less required to take a 'splitting' rather than a 'lumping' approach to polysemy." (Fillmore \& Baker 2010: 333). Da eine lexikographische Beschreibungseinheit bei FrameNet eine LE, also ein „pairing of a word with a sense“ (Fillmore, Johnson \& Petruck 2003: 235) ist, wird Splitting durch den Ansatz verschiedener LE ausgedrückt (vgl. Unterabschnitt 2.1.2). Das oben diskutierte Beispiel von erscheinen illustriert dies: Die vier in den Belegen (10)-(13) sichtbaren Lesarten dieses Wortes werden durch vier verschiedene LE und somit vier verschiedene Frames erfasst. Wie die lexikographische Erfassung eines polysemen Wortes steht die konstruktikographische Erfassung einer polysemen syntaktischen Konstruktion nun ebenso vor der Frage, ob ein Splitting oder Lumping ihrer unterschiedlichen Lesarten angebracht ist (vgl. dazu auch Zeschel \& Proost 2019: 137).

Das FrameNet-Konstruktikon verfährt im Falle der way-Konstruktion, wie bereits in Unterabschnitt 2.3.2 angedeutet, analog zum lexikalischen FrameNet. Für die beiden Lesarten der Konstruktion werden jeweils eigene Konstruktionseinträge angelegt: way_manner und way_means. Hinzu kommt eine neutrale Lesart: way_neutral. Die von Israel (1996: 218), Perek (2018: 68-69) und McColm (2019: 39) angenommene, incidental'-Lesart wird im FrameNet-Konstruktikon nicht be- 
rücksichtigt. Grundsätzlich aber wird sichtbar, dass das FrameNet-Konstruktikon analog zum lexikalischen FrameNet die Strategie verfolgt, polyseme Konstruktionen gemäß des Splitting-Ansatzes durch unterschiedliche Konstruktionseinträge darzustellen. Mit anderen Worten: Eine polyseme Konstruktion muss konstruktikographisch anders behandelt werden als eine Konstruktion ohne Polysemie.

Die Maxime, dass konstruktionelle Polysemie für die konstruktikographische Erfassung einer Konstruktion von Relevanz ist, leitet über zu der Frage, die den Parameter der konstruktionellen Polysemie motiviert: Wie lässt sich konstruktionelle Polysemie auf der Basis des konstruktionssemantischen Modells aus Kapitel 4 auffassen? Für eine vorläufige Antwort lassen sich aus einem konstruktionssemantischen Blickwinkel zwei grundsätzliche Arten von Polysemie syntaktischer Konstruktionen charakterisieren.

a) Dieselbe Konstellation von Strukturelementen (KE, KEE und KorE) ist mit unterschiedlichen Frames assoziiert, die als Konstruktions-Frames in den Konstrukten evoziert werden.

b) Der Konstruktions-Frame bleibt über alle Konstrukte (mehrerer Lesarten) hinweg invariant, semantische Unterschiede in den Konstrukten kommen bei gleicher Konstellation von KE, KEE und KorE durch andere systematische Verhältnisse zustande, insbesondere durch unterschiedliche lexikalische Frames und innerhalb dieser wiederum unterschiedlicher lexikalischer Bedeutungen.

Die Frage, ob bei der Polysemie einer syntaktischen Konstruktion ein Splittingoder Lumping-Ansatz verfolgt werden muss, richtet sich danach, welche dieser beiden Arten von Polysemie vorliegt. Ich möchte beide im Folgenden in den Unterabschnitten 5.2.1 und 5.2.2 zunächst näher charakterisieren und aufzeigen, warum für Polysemie im Sinne von a ein Splitting-Ansatz gerechtfertigt ist und für Polysemie im Sinne von b ein Lumping-Ansatz verfolgt werden sollte. Die drei untersuchten Konstruktionen, insbesondere die reflexive Bewegungskonstruktion und die reflexive Partikelverbkonstruktion, sind indes von Polysemie im Sinne von b betroffen, weshalb ich am Beispiel der reflexiven Bewegungskonstruktion in Unterabschnitt 5.2.3 den konstruktionssemantischen Umgang mit ihrer Polysemie stellvertretend für ihre beiden verwandten Konstruktionen erläutere. Auf einige darüber hinausreichende Prinzipien bei der Differenzierung der vier Lesarten gehe ich in Unterabschnitt 5.2.4 ein. 


\subsubsection{Polysemie durch unterschiedliche Konstruktions-Frames}

Die Polysemie einer Konstruktion durch unterschiedliche Konstruktions-Frames $\mathrm{zu}$ verstehen ist ein frame-semantisch simpler Weg. Ihn beschreiten etwa Perek \& Patten (2019: 374-382) bei ihrer Analyse der englischen V-that-Konstruktion, für die sie zahlreiche Frames identifizieren, die mit dem ihr zugrunde liegenden grammatischen Muster assoziiert sind. Über die Differenzierung dieser Lesarten hinaus lassen sich durch die Frame-zu-Frame-Relationen, in denen die identifizierten Frames stehen, jeweils übergeordnete Frames auffinden, die Generalisierungen der Frames auf einer niedrigeren Abstraktionsebene darstellen und das Inventar an Lesarten einer Konstruktion weiter abstrahieren. So ermitteln Perek \& Patten (2019: 375-379) schlussendlich vier Frames für die V-that-Konstruktion, die mit deren generellen Lesarten korreliert werden können und jeweils ein Netzwerk weiterer untergeordneter Frames, die entsprechende Teil-Lesarten abbilden, um sich herum aufspannen.

Ein Beispiel für konstruktionelle Polysemie durch unterschiedliche Konstruktions-Frames lässt sich unter den von mir untersuchten Konstruktionen nicht finden. Stattdessen soll eine andere, strukturell von ihnen vollkommen verschiedene Konstruktion diese Art von Polysemie illustrieren: die Subjekt-AuxiliarInversion. ${ }^{15}$ Für sie zählt Goldberg (2006: 177) neun Lesarten für das Englische auf (vgl. auch Goldberg \& Del Giudice 2005). Für mindestens vier dieser Lesarten lassen sich deutsche Belege im DWDS-Kernkorpus 21 finden, ${ }^{16}$ die ein finites Auxiliarverb am Satzanfang enthalten. ${ }^{17}$ Wenngleich etwa Bäckström, Lyngfelt \& Sköldberg (2014: 29) bezweifeln, dass sich die Subjekt-Auxiliar-Inversion unter Rückgriff auf Frames beschreiben lässt und für sie eher, grammatische‘ Bedeutungen angesetzt werden müssen, lassen sich diesen vier Lesarten tatsächlich

15 Es soll nicht unerwähnt bleiben, dass die Frage, ob der Subjekt-Auxiliar-Inversion überhaupt semantische Eigenschaften zukommen und sie deshalb als Konstruktion im konstruktionsgrammatischen Sinne zu betrachten ist, kontrovers diskutiert wird. Als prominentester Gegner einer solchen Annahme dürfte - aus konstruktionsgrammatischer Sicht besonders interessant - Fillmore (1999) gelten. Eine explizite Gegenposition zu Goldberg (2006: 166-182) nehmen Borsley \& Newmeyer (2009) ein, sprachtheoretische Gegenargumente diskutiert Jackendoff (2007: 367-368). Eine Diskussion über mögliche ,bedeutungslose‘ Konstruktionen bietet, unter Einbezug der Position von Fillmore, Lee-Goldman \& Rhomieux (2012: 325-328) sowie des Ansatzes von Goldberg (2006: 166-182) zur Subjekt-Auxiliar-Inversion, Hilpert (2019: 50-57).

16 Zur Datengrundlage vgl. Unterabschnitt 3.4.3.

17 Daneben scheint es für das Deutsche über die in Tabelle 5.1 aufgelisteten Lesarten für das Englische hinaus noch weitere Lesarten zu geben. Diese müssten in einer dezidierten Studie ergründet werden, ebenso wie die Frage, welche Konstruktions-Frames für sie angesetzt werden können. 
vier FrameNet-Frames zuordnen, die als Konstruktions-Frames infrage kommen. Eine entsprechende Übersicht ist in Tabelle 5.1 dargestellt.

Tab. 5.1: Lesarten der englischen Subjekt-Auxiliar-Inversion nach Goldberg (2006: 177) und ihre Entsprechungen als Konstruktions-Frames

\begin{tabular}{lll}
\hline Lesart nach Goldberg & Belege & Konstruktions-Frame \\
\hline A. Ja-/Nein-Fragen & $(14)$ & Questioning \\
$\begin{array}{l}\text { B. Kontrafaktische Konditionale } \\
\text { C. Initiales negatives Adverb }\end{array}$ & & \\
$\begin{array}{l}\text { D. Negatives Konjunkt } \\
\text { E. Komparative }\end{array}$ & & \\
$\begin{array}{l}\text { F. Wünsche und Flüche } \\
\text { G. w-Fragen }\end{array}$ & $(16)$ & $\begin{array}{l}\text { Desiring } \\
\text { Questioning }\end{array}$ \\
$\begin{array}{l}\text { H. Positives Konjunkt } \\
\text { I. Exklamativa }\end{array}$ & $(17)$ & Experiencer_obj \\
\hline
\end{tabular}

Die Belege unter (14) sind Beispiele für eine Lesart der Subjekt-Auxiliar-Inversion, der der Konstruktions-Frame Questioning zugeordnet werden kann.

(14) a. Hat unser Großvater keine Aufnahme von ihr allein gemacht? (Beyer, Marcel: Spione, Köln: DuMont 2000, S. 37)

b. War ich jetzt ein Rebell? (Goosen, Frank: Liegen lernen, Frankfurt am Main: Eichborn AG 2000, S. 58)

c. Sind Sie ein Perfektionist? (Die Zeit, 05.01.2000, Nr. 2)

Belegen wie denen in (15), die über eine (allerdings nicht notwendigerweise kontrafaktische) konditionale Lesart im Sinne von Goldberg (2006: 177) verfügen, kann der Frame Condi tional_scenario als Konstruktions-Frame zugeordnet werden.

(15) a. Würden sich die Erwachsenen nicht das Maul zerreißen, wären auch ihre Kinder still. (Beyer, Marcel: Spione, Köln: DuMont 2000, S. 20)

b. Wird das Projekt aber lanciert und scheitert, ist der Erfolg von 30 Jahren erfolgreicher Arbeit in Gefahr. (Die Zeit, 05.01.2000, Nr. 2)

c. Ist er bei seiner zweiten Frau, verrät er seine frühere Familie. (Beyer, Marcel: Spione, Köln: DuMont 2000, S. 106)

Belegen wie denen in (16), die in funktionalgrammatischer Terminologie Beispiele für typische Optativ- bzw. ,Wunsch'-Sätze sind (vgl. z.B. Hentschel \& Weydt 2013: 
380; Duden 2016: 904-905), kann Desiring als Konstruktions-Frame zugeordnet werden. ${ }^{18}$

(16) a. Wäre nur ein Einheimischer dabei! (Die Zeit, 17.02.2000, Nr. 8)

b. Hättest du die Verabredung mit Kári Stefánsson doch nur schon für morgen getroffen! (Venske, Regula: Marthes Vision, Frankfurt am Main: Eichborn Verlag 2006, S. 50)

c. Sei ja ein Schicksalsschlag, murmelte er, da wolle er Haueisen Scherereien ersparen. (Koneffke, Jan: Paul Schatz im Uhrenkasten, Köln: DuMont Buchverlag 2000, S. 263)

Als vierte Lesart kann schließlich Belegen mit einer Exklamativ-Lesart wie denjenigen in (17) der Frame Experiencer_obj als Konstruktions-Frame zugeordnet werden (vgl. dieselbe Analyse von Ziem \& Ellsworth 2016: 159-160, 164).

(17) a. War das ein Schock! (Schwanitz, Dietrich: Männer, Frankfurt a. M.: Eichborn 2001, S. 87)

b. Hast du mir einen Schrecken eingejagt! (Schwanitz, Dietrich: Männer, Frankfurt a. M.: Eichborn 2001, S. 229)

c. Bist du verrückt! (Kopetzky, Steffen: Grand Tour, Frankfurt am Main: Eichborn 2002, S. 414)

Unterschiedlichen Lesarten derselben Konstruktion wie der Subjekt-AuxiliarInversion unterschiedliche Konstruktions-Frames zuzuordnen entspricht ziemlich genau der Praxis des lexikalischen FrameNets, polyseme Wörter als unterschiedliche LE, die unterschiedliche Frames evozieren, zu erfassen. Aus konstruktionssemantischer Sicht ist die semantische Variabilität einer polysemen Konstruktion deutlicher kaum zu erfassen. Für jede der Lesarten, die mit einem Frame korreliert, wäre demnach ein eigener Konstruktionseintrag anzulegen ${ }^{19}$ - genauso verfährt im Übrigen das FrameNet-Konstruktikon für die englische

18 Offen bleibt freilich, inwiefern Frames wie Questioning und Desiring als KonstruktionsFrames der Subjekt-Auxiliar-Inversion die Grenze zu interaktionalen Frames im Sinne von Fillmore (1982a: 117) überschreiten (vgl. auch Unterabschnitte 2.2.3 und 2.3.2). Vgl. dazu die Bemerkungen von Boas, Lyngfelt \& Torrent (2019: 47) zu Imperativkonstruktionen, ,which, while obviously related to the Request frame, hardly evoke it the same way words like order and command do; rather than referring to requests, like these words do, imperative cxns are used to perform them.“

19 Diese Form von Polysemie begründet damit ein eigenes Konstruktionsnetzwerk, wie es Goldberg (1995: 75-77) für ihre Polysemie-Relation zwischen Konstruktionen annimmt. Demgegenüber begründet Polysemie durch variierende lexikalische Frames und Bedeutungen (Unterabschnitt 5.2.2) kein solches Konstruktionsnetzwerk, weshalb in diesem Fall keine einzelnen Konstruktionseinträge für jede Lesart angelegt werden müssen. In ihrer Gegenüberstellung von Gold- 
Subjekt-Auxiliar-Inversion, ohne dabei jedoch Frames einzubeziehen. ${ }^{20}$ Unterschiedliche Lesarten durch unterschiedliche Konstruktions-Frames zu erfassen ist somit ein genuiner Splitting-Ansatz. Die reflexive Bewegungskonstruktion hingegen erlaubt wie die reflexive Partikelverbkonstruktion und die reflexive WegKonstruktion ein solches Verfahren gerade nicht, da der Konstruktions-Frame Motion über alle Konstrukte (mehrerer Lesarten) hinweg invariant bleibt. Die beobachtbaren semantischen Nuancen, die zur Annahme von Polysemie führen, sind grundsätzlich anderer Natur und erfordern deshalb auch einen anderen konstruktikographischen Ansatz: einen Lumping-Ansatz.

\subsubsection{Polysemie durch variierende lexikalische Frames und Bedeutungen}

Gegenüber der Feststellung von Polysemie durch unterschiedliche KonstruktionsFrames, die einen Splitting-Ansatz rechtfertigt, ist die Annahme von Polysemie durch variierende lexikalische Frames und lexikalische Bedeutungen subtiler, da in diesem Fall semantische Unterschiede in den Konstrukten der Konstruktion nicht bereits durch unterschiedliche Konstruktions-Frames erfassbar sind. Wenn der Konstruktions-Frame über alle Konstrukte (mehrerer Lesarten) hinweg invariant bleibt, muss konstruktionelle Polysemie durch andere Zusammenhänge zustande kommen. Eine wesentliche Triebfeder hierfür sind die lexikalischen Frames und die aus ihnen resultierenden lexikalischen Bedeutungen, die in die Konstrukt-Frames bzw. Konstruktbedeutungen eingehen. ${ }^{21}$ Die auf Ziem (2020b: 44-48) zurückgehende Unterscheidung zwischen lexikalischen Frames und lexikalischen Bedeutungen (Unterabschnitt 4.1.1) wird hier besonders relevant: Lesarten können zwar einerseits über unterschiedliche lexikalische Frames differenziert werden, andererseits müssen aber auch unterschiedliche lexikalische

bergs Polysemie-Relation mit der Idee der Familienähnlichkeiten scheinen Proost \& Winkler (2015: 7-8) diese Form von Polysemie nicht zu erwägen.

20 Die einzelnen Einträge haben die Namen Subject_auxiliary_inversion.closed_interrogative, Subject_auxiliary_inversion.conditional, Subject_auxiliary_inversion.emphatic_negative_imperative, Subject_auxiliary_inversion.exclamation und Subject_auxiliary_inversion.optative. Hinzu kommt eine allgemeine Subject_auxiliary_inversion, die den anderen Konstruktionen übergeordnet ist (vgl. http://sato.fm.senshu-u.ac.jp/frameSQL/cxn/CxNeng/cxn00/21colorTag/index. html, zuletzt abgerufen am 07.09.2021).

21 Die Relevanz der Slots eines Frames einerseits und der Füllwerte bestimmter Slots andererseits führt bereits Martin (2001: 69-73) als zwei Möglichkeiten der Differenzierung von (lexikalischer) Polysemie auf. Die in diesem Unterabschnitt diskutierte Art von konstruktioneller Polysemie und deren Differenzierung über lexikalische Bedeutungen (also Standardwerten innerhalb lexikalischer Frames) kommt diesem Ansatz also prinzipiell nahe. 
Bedeutungen, die auf demselben Frame beruhen, für die Polysemie einer Konstruktion verantwortlich gemacht werden. Die drei untersuchten Konstruktionen, gerade die reflexive Bewegungskonstruktion und die reflexive Partikelverbkonstruktion mit ihren Lesarten ,manner', ,means' und ,incidental' sowie einer neutralen Lesart, sind hierfür ein Beispiel. Insbesondere die ,manner'-Lesart soll in dieser Hinsicht in den Unterabschnitten 5.2.3 und 5.2.4 analysiert werden.

Konstruktionelle Polysemie über lexikalische Frames und lexikalische Bedeutungen zu differenzieren, ist ein Verfahren, das gewissermaßen bereits Perek (2014, 2015: 111-142) anwendet, allerdings aus stärker methodischen Gründen:22 Da eine Kollostruktionsanalyse zur englischen Konativkonstruktion keine eindeutige Distrubution von Verben ergibt, die auf eine einheitliche Konstruktionsbedeutung schließen lassen könnten, führt er nach Verbklassen getrennte Kollostruktionsanalysen durch (dazu auch Unterabschnitt 8.5.1). Ergebnis ist ein deutlich homogeneres Bild:

[F]ocusing on a particular class of verbs clearly captures what the semantic contribution of the construction is for this particular class. Thus, a collexeme analysis at the level of individual verb classes seems to be a promising approach. (Perek 2014: 72)

In Anlehnung an Croft (2003: 53-59) plädiert Perek (2014: 71) sodann dafür, nicht eine Konstruktion mit unterschiedlichen Lesarten anzusetzen, sondern diese als mehrere verbklassen-spezifische Konstruktionen zu betrachten. Dieser Ansatz ist nicht nur interessant, weil er lexikalische Bedeutungen auf der Basis von Verbklassen (vergleichbar mit den LE eines lexikalischen Frames, vgl. Baker \& Ruppenhofer 2002; Boas 2006) zum Ausgangspunkt für die Differenzierung unterschiedlicher Lesarten einer Konstruktion macht, sondern gleichzeitig von einem radikalen Splitting-Ansatz ausgeht, der bis zur Ablehnung von Polysemie und der Annahme verbklassen-spezifischer Konstruktionen reicht. ${ }^{23}$ Bevor ich für die reflexive Bewegungskonstruktion aufzeigen möchte, wie lexikalische Frames und lexi-

22 Dezidiert an lexikalische Frames scheint auch Croft (2012: 374) zu denken: „Other constructions, [...], would have a very wide range of constructional polysemy. These constructions allow alternative construals in many different frames, depending on the force-dynamic potential of the verb that is put in the construction."

23 Indes analysiert auch Goldberg (1995: 210-212, 1996: 42-44) die unterschiedlichen Lesarten der way-Konstruktion mittels ihrer Polysemie-Relation zwischen Konstruktionen (vgl. Goldberg 1995: 75-77) und damit als zwar in einem Konstruktionsnetzwerk zusammenhängende, aber dennoch unterscheidbare Konstruktionen. Der hier vertretene Ansatz für die reflexive Bewegungskonstruktion und ihre verwandten Konstruktionen richtet sich also gleichsam gegen diese im Gegensatz zu Croft (2003: 53-59) und Perek (2014: 71) zwar weniger radikale, aber ebenso als Splitting-Ansatz zu klassifizierende Vorgehensweise Goldbergs. 
kalische Bedeutungen ihre Lesarten differenzieren, möchte ich kurz auf die Probleme eines radikalen Splitting-Ansatzes, wie ihn Croft und Perek vertreten, eingehen und begründen, warum dieser für die drei untersuchten Konstruktionen unangebracht erscheint. Gleichzeitig sollen diese Argumente die Begründung dafür liefern, warum ein Lumping-Ansatz für die drei untersuchten Konstruktionen sinnvoll erscheint, zumal die Art konstruktioneller Polysemie durch variierende lexikalische Frames und lexikalische Bedeutungen einen solchen Ansatz nahelegt.

Zwei Argumente sprechen gegen die Angemessenheit eines Ansatzes, der konstruktionelle Polysemie grundsätzlich ablehnt und unterschiedliche Konstruktionen postuliert, seien sie spezifisch auf Verbklassen oder lexikalische Frames einerseits ausgerichtet oder durch lexikalische Bedeutungen andererseits differenziert. Das erste Argument liegt in dem schon mehrfach betonten Fakt, dass der Konstruktions-Frame über alle Konstrukte der Konstruktion invariant bleibt. Konstruktionen, die formal identisch sind und über denselben Konstruktions-Frame verfügen, müssen fast zwangsläufig als zusammengehörig betrachtet werden, da der Konstruktions-Frame ja gerade diese Zusammengehörigkeit kodiert (vgl. die Ausführungen zu Familienähnlichkeiten in Unterabschnitt 3.2.3). Aufgrund dessen erscheint es auch vor dem Hintergrund der Vorstellung eines Konstruktikons als Konstruktionsnetzwerk unangebracht, Konstruktionen, die eine solche Verwandtschaft aufweisen, als unverbunden zu betrachten oder sie auch nur in einen Splitting-Ansatz zu überführen und unterschiedliche Konstruktionseinträge für sie anzusetzen. ${ }^{24}$ Das zweite Argument besteht darin, dass die Unterschiede in den Lesarten ja gerade äußerst subtiler Art sind, da sie lediglich durch unterschiedliche lexikalische Frames oder lexikalische Bedeutungen innerhalb eines lexikalischen Frames zustande kommen. Ein radikaler Splitting-Ansatz empfiehlt sich deshalb nur bei Konstruktionen, deren Lesarten über unterschiedliche Konstruktions-Frames differenziert sind, wie etwa der Subjekt-Auxiliar-Inversion (Unterabschnitt 5.2.1). Bleibt der Konstruktions-Frame jedoch invariant und sind es lediglich die lexikalischen Frames und lexikalischen Bedeutungen, die für Polysemie sprechen, erscheint es kaum gerechtfertigt, unterschiedliche Konstruktionen anzusetzen und einen Splitting-Ansatz zu verfolgen.

24 Perek (2014) geht allerdings nicht so weit zu behaupten, den von ihm ermittelten verbklassenspezifischen Konstruktionen komme keine Verwandtschaft in diesem Sinne zu, jedoch unternimmt er keine Bestrebungen, sie als Netzwerk zu modellieren. Die betonte Relevanz der Notwendigkeit niedrigerer Abstraktionsebenen, auf denen diese verbklassen-spezifischen Konstruktionen liegen (vgl. Perek 2014: 83) bringt eine netzwerkartige Modellierung jedoch nicht in jedem Fall mit sich. 
Es muss also in jedem Fall abgewogen werden, ob es sich bei der Differenzierung einer polysemen Konstruktion (nach welchen Kriterien auch immer) um mehrere voneinander unterschiedene (Teil-)Konstruktionen handelt, für die konstruktikographisch ebenso mehrere Konstruktionseinträge angelegt werden müssen, oder ob die semantischen Nuancen tatsächlich für ein Postulat mehrerer unterschiedlicher Konstruktionen ausreichen, wie Imo (2011) kritisiert:

Das Problem bei dieser Kombination - dass eine phonologische Form mehrere Funktionen oder Bedeutungen tragen kann - besteht darin, dass man nicht immer dafür argumentieren kann, dass es sich dabei um verschiedene Konstruktionen (also Zeichen) handelt, sondern dass diese Bedeutungen „,irgendwie“ alle Bestandteil der „Konstruktion“ sein können und je nach Kontext aktiviert oder deaktiviert sind. Es handelt sich dabei um solche Formen, die viele differenzierte Bedeutungsvarianten aufweisen, die aber selten oder nie in einer reinen, prototypischen Form vorkommen, sondern immer in unterschiedlichen Anteilen gemischt sind, also in der aktuellen Verwendung selbst Polysemie aufweisen. (Imo 2011: 122-123)

Die Frage, wann Splitting aufhören muss und wann Lumping angebracht ist, ist somit eine Kernfrage der Diskussion um konstruktionelle Polysemie und betrifft grundsätzlich den Grad der konstruktikographischen Generalisierung (dazu Unterabschnitt 7.2.2). Gewissermaßen kritisiert Imo (2011) in diesem Sinne den Ansatz zu vieler unterschiedlicher Konstruktionen, die nur durch subtile Unterschiede (im vorliegenden Fall: lexikalische Frames und lexikalische Bedeutungen) gekennzeichnet sind, weshalb der Abstraktionsgrad nicht zu niedrig werden sollte:

Dieses Problem [der Polysemie von Konstruktionen, A.W.] lässt sich nicht dadurch lösen, dass man der Konstruktionsbeschreibung mehr Einträge zu weiteren Informationstypen zugesteht: Auch durch zusätzliche Informationen über Kontext, Prosodie o.Ä. lassen sich keine systematisch strukturierten Konstruktionen erstellen - bestenfalls würde eine höchst detaillierte Ausweitung dazu führen, dass Konstruktionen zu Konstrukten („constructs“) werden, d.h. also dass nur noch die „Tokens“ gezählt aber keine „Types“ mehr erstellt werden könnten. Eine Grammatik, die nur noch aus „Tokens“ besteht, ist aber keine mehr. (Imo 2011: 123)

Die drei untersuchten Konstruktionen, insbesondere die reflexive Bewegungskonstruktion und die reflexive Partikelverbkonstruktion, sind Fälle von subtiler konstruktioneller Polysemie, die jeweils durch unterschiedliche lexikalische Frames und lexikalische Bedeutungen zustande kommt. Deshalb bietet es sich an, ihre bereits in der Literatur zur way-Konstruktion ausführlich thematisierten Lesarten, 
,manner‘, ,means' und ,incidental' sowie eine neutrale Lesart, entsprechend zu analysieren. ${ }^{25}$

\subsubsection{Differenzierung von neutraler, ,manner--, ,means`- und ,incidental'-Lesart}

Hinsichtlich der konstruktionellen Polysemie der drei untersuchten Konstruktionen möchte ich für einen Lumping-Ansatz plädieren, der in Anlehnung an die Umsetzung des FrameNet-Konstruktikons und die aktuellere Forschung zur wayKonstruktion (v.a. Perek 2018: 68-69; McColm 2019: 39) die Lesarten ,manner“, ,means' und ,incidental' sowie eine neutrale Lesart der Konstruktion unterscheidet (vgl. dazu schon Unterabschnitt 3.1.3), diese aber nicht als grundsätzlich voneinander verschiedene Konstruktionen betrachtet und deshalb auch keine eigenen Konstruktionseinträge für jede Lesart vorsieht (vgl. auch Unterabschnitt 7.2.2). Obwohl ich im Folgenden vor allem die reflexive Bewegungskonstruktion heranziehe, gelten die Ergebnisse für alle drei Konstruktionen, da die vier Lesarten darüber hinaus insbesondere für die reflexive Partikelverbkonstruktion belegt sind.

Zunächst seien die vier Lesarten noch einmal an einigen Beispielen illustriert. Die Konstrukte in (18) sind Beispiele für die neutrale Lesart der reflexiven Bewegungskonstruktion, die weder nach einem ,manner'- noch nach einem ,means'Aspekt differenziert ist und in dem lexikalischer Frame und Konstruktions-Frame nicht derart distinkt sind, dass eine, incidental'-Lesart entstehen könnte. Da die lexikalischen Frames und die von ihnen ausgehenden lexikalischen Bedeutungen entscheidend sind, sind Erstere in jedem Konstrukt annotiert.

(18) a. Auch ich [Motion begebe] mich in den Garten und pflücke mir ein bescheidenes Sträußchen kriechender Kapuzinerkresse. (Noll, Ingrid: Ladylike, Zürich: Diogenes 2006, S. 137)

b. Ein Soldat [Body_movement beugte] sich in Pauls Haushaltsbuch und kratzte sich ratlos am Kopf. (Koneffke, Jan: Paul Schatz im Uhrenkasten, Köln: DuMont Buchverlag 2000, S. 232)

c. Die ersten Menschen, die sie traf, wagte sie noch nicht anzuschauen, sie senkte den Kopf und [self_motion drückte] sich an Hauswände oder in Toreinfahrten, doch bald merkte sie, dass niemand sie beachtete. (Pressler, Mirjam: Malka Mai, Weinheim Basel: Beltz \& Gelberg 2001, S. 114)

25 Einen anderen Weg gehen für die way-Konstruktion Traugott \& Trousdale (2013: 77), die annehmen, dass die Lesarten ,are polysemous subschemas of the superordinate way-construction schema.“ 
Die Konstrukte in (19) zeigen die ,manner'-Lesart, in der die ,Art und Weise' der ,Bewegung، ausgedrückt wird, genauer: bereits als Standardwert in der lexikalischen Bedeutung der LE, die den jeweiligen lexikalischen Frame evoziert, angelegt ist.

(19) a. General Motors geht es mit seiner Tochter Opel kaum besser, Fiat [self_motion robbt] sich mühsam aus den roten Zahlen, und Renault hat die Übernahme von Nissan noch längst nicht bewältigt. (Die Zeit, 24.02.2000, Nr. 9)

b. Er [Body_movement warf] sich in Hosen und Hemd, schlich aus Haueisens Wohnung, und als er im Hof ankam, atemlos, lehnte sie an einem Baum und schaute abwechselnd zu Paul und zu Boden, verlegen, als handle es sich um einen Irrtum. (Koneffke, Jan: Paul Schatz im Uhrenkasten, Köln: DuMont Buchverlag 2000, S. 194)

c. Er [Body_movement zitterte] sich in einen Dämmerschlaf, von dem er nicht wußte, ob er zehn Minuten dauerte oder drei Stunden. (Glavinic, Thomas: Die Arbeit der Nacht, München Wien: Carl Hanser Verlag 2006, S. 167)

Die Konstrukte in (20) zeigen die ,means‘-Lesart, die die ,Mittel', durch welche die ,Bewegung‘ ermöglicht wird, kodiert.

(20) a. Mit jedem Tag deutlicher [Emptying schält] sich ein unbekanntes Wesen aus der groben Form. (Riedel, Susanne: Eine Frau aus Amerika, Berlin: Berlin Verlag 2003, S. 11)

b. Der Fabrikant breitete sein Schweigen wieder aus, der Offizier [Manipulate_into_doing mogelte] sich aus seinem Sitz. (Düffel, John von: Vom Wasser, München: dtv 2006, S. 56)

c. Man begann bei den Gesetzen der Körperwelt, [work arbeitete] sich empor zur Psychologie, zu den Gesetzen des Empfindens und Denkens, Ausflüge in die Schöne Literatur waren vorgesehen, sie ermöglichten den Übergang zum Feinseelischen und zu den letzten Fragen, Gott und Unsterblichkeit. (Safranski, Rüdiger: Friedrich Schiller, München Wien: Carl Hanser 2004, S. 46)

Schließlich zeigen die Konstrukte in (21) die ,incidental'-Lesart, bei der zu erkennen ist, dass sich lexikalischer Frame (hier jeweils Dead_or_alive, Make_noise und Daring) und Konstruktions-Frame (Motion) voneinander unterscheiden, jedoch nicht in einer kausalen (,means'-)Beziehung zu einander stehen, und die lexikalische Bedeutung jeweils nicht hinsichtlich eines ,manner'-Aspekts spezifiziert ist. Wie noch zu zeigen sein wird, entsteht die, incidental'-Lesart insbeson- 
dere dann, wenn im Konstrukt-Frame der Anteil des lexikalischen Frames am geringsten ist, wenn also sowohl die Instanz des KEE als auch das KtE des KE WEG allein durch den Konstruktions-Frame motiviert sind und nicht einfach durch den lexikalischen Frame (Unterabschnitte 6.2.1 und 6.3.1) oder doppelt durch lexikalischen Frame und Konstruktions-Frame (Unterabschnitte 6.2.3 und 6.3.3). Dies ist ein Indiz für die größtmögliche Distinktivität von Konstruktions-Frame und lexikalischem Frame.

a. Man [Dead_or_alive lebte] sich aus der herausgerissenen Zeit, in der man saß, zurück in die Erinnerung ans Zuhause von damals und voraus in die Hoffnung, bald heimzukehren. (Müller, Herta: Der König verneigt sich und tötet, München: Carl Hanser Verlag 2003, S. 42)

b. „ ... für die vielen Toten und Verwundeten hergeschickt worden waren, nachgerückt wie Kegel im Spiel“, machte der Vater seinen Fehler wieder gut, ,junge Kerle, gerade Abitur gemacht, [Make_noise heulten] sich auf ihren Strohschütten in den Erdbunkern, wo wir uns zusammendrängten, in den Schlaf. (Hahn, Ulla: Unscharfe Bilder, München: Deutsche Verlags-Anstalt 2003, S. 103)

c. Es [Daring wagten] sich keine SA-Leute ins Scheunenviertel, um Steine in Mosche Sternkukkers Buchladen zu werfen. (Koneffke, Jan: Paul Schatz im Uhrenkasten, Köln: DuMont Buchverlag 2000, S. 26)

Bereits in Unterabschnitt 3.1.3 habe ich darauf hingewiesen, dass eine Unterscheidung zwischen diesen Lesarten bisweilen schwierig ist. Deshalb soll vor einer eingehenderen Diskussion nicht unerwähnt bleiben, dass die Unterscheidung dieser Lesarten bereits für die way-Konstruktion auf Kritik gestoßen ist. So kritisiert Luzondo Oyón (2013: 359-360), dass die Entscheidung darüber, ob in einem gegebenen Konstrukt die ,manner'- oder ,means'-Lesart vorliegt, stark kontextabhängig ist, die Lesarten eher als zwei verschiedene Perspektiven auf dasselbe Ereignis zu verstehen und grundsätzlich nur schwierig auseinanderzuhalten sind. Sie zieht daraus den Schluss, dass die Trennung der Lesarten aufgrund dieser Befunde ohne Mehrwert ist:

In any case, whenever an activity is performed, there is always some intrinsically related manner of carrying it out, and thus, distinguishing between means and manner is rather irrelevant. (Luzondo Oyón 2013: 360) 
Den Kritikpunkt der nur kontextabängigen Differenzierbarkeit zwischen der ,manner'- oder ,means'-Lesart greift auch Smirnova (2018) auf und weist dabei auf einen möglichen alternativen Bezugspunkt der Analyse hin: ${ }^{26}$

Was darüber entscheidet, ob die eine oder die andere Lesart vorliegt, sind nicht die konstruktionsinternen Aspekte, sondern vielmehr die konstruktionsexternen, d. h. es handelt sich um bestimmte kontextuell präsente Informationen. (Smirnova 2018: 36-37)

Diese angenommene Kontextabhängigkeit der Lesarten ist ein Hinweis auf die Subtilität, mit der sie in den Konstrukten wirksam werden: Die unterschiedlichen Lesarten lassen sich gerade nicht, wie bei der Subjekt-Auxiliar-Inversion, auf unterschiedliche Konstruktions-Frames zurückführen - dies würde einen Splitting-Ansatz rechtfertigen (Unterabschnitt 5.2.1). Da jeder der drei untersuchten Konstruktionen nur ein einziger Konstruktions-Frame - Motion - zukommt, spielen vielmehr Faktoren eine Rolle, die jenseits des Konstruktions-Frames liegen. Berücksichtigt werden müssen hier also lexikalische Frames und insbesondere lexikalische Bedeutungen, die, wenn sie ursächlich für konstruktionelle Polysemie sind, einen Lumping-Ansatz für die Konstruktion begründen (wie in Unterabschnitt 5.2.2 argumentiert).

Sieht man sich für die way-Konstruktion an, wodurch ihre Lesarten ,manner“, ,means', ,incidental' sowie eine neutrale Lesart konstituiert werden, so zeigt sich, dass das Verhältnis zwischen Konstruktions-Frame und lexikalischen Frames sowie insbesondere lexikalischen Bedeutungen einen entscheidenden Einfluss besitzt. Indes lassen sich nicht nur für die way-Konstruktion Beispiele für solche Lesarten finden. Ganz grundsätzlich zählt Goldberg (1997: 386-395) etwa die ,means“Lesart zu den wesentlichen Relationen zwischen einer lexikalischen Bedeutung und einer ,Konstruktionsbedeutung،. Auch die ,manner'-Lesart gehört laut Goldberg (1998: 46-49) unter dem Begriff co-occurring activity dazu. Dabei sind, wie Goldberg illustriert, solche semantischen Relationen zwischen lexikalischer Bedeutung und Konstruktionsbedeutung nicht nur der way-Konstruktion vorbehalten, sondern lassen sich für eine ganze Reihe an Konstruktionen finden.

Den Gedanken, dass eine bestimmte Lesart an das Verhältnis zwischen Konstruktions-Frame sowie lexikalischen Frames und lexikalischen Bedeutungen geknüpft ist, möchte ich für die reflexive Bewegungskonstruktion stellvertretend für ihre verwandten Konstruktionen weiterverfolgen und zeigen, dass sich die unterschiedlichen Lesarten der Konstruktionen aufgrund der Verteilung

26 Sowohl Luzondo Oyón (2013) als auch Smirnova (2018) berücksichtigen die ,incidental'Lesart ebenso wie eine neutrale Lesart nicht, weshalb sie lediglich die Unterscheidung zwischen ,manner' und ,means‘ diskutieren. 
lexikalischer Frames und lexikalischer Bedeutungen differenzieren lassen. Zusammengefasst lassen sich die Zusammenhänge in vier Prinzipien festhalten, die in den vier zu Beginn dieses Unterabschnitts zitierten Gruppen von Konstrukten, (18)-(21), bereits sichtbar werden.

1. Konstrukte, deren Konstrukt-Frames durch den lexikalischen Frame allein konstituiert werden, erhalten in der Regel eine neutrale oder eine ,manner'Lesart. Ein Beispiel dafür sind die Konstrukte in (18), die die lexikalischen Frames Motion, Body_movement und Self_motion evozieren.

2. Ob bei einem lexikalischen Frame eine neutrale oder eine ,manner'-Lesart vorliegt, entscheidet sich durch die lexikalische Bedeutung der LE, die den lexikalischen Frame evoziert. Hierfür sind die Konstrukte in (19) ein Beispiel, da sie ebenfalls Frames wie Self_motion (robben/crawl.v) und Body_movement (werfen/throw.v, zittern/shake.v) als lexikalische Frames evozieren, allerdings andere lexikalische Bedeutungen mit sich bringen.

3. Die ,means'-Lesart kommt insbesondere dann zustande, wenn der KonstruktFrame aus lexikalischem Frame und Konstruktions-Frame zugleich konstituiert wird. In den Konstrukten in (20) etwa werden die lexikalischen Frames Emptying (durch die LE schälen/peel.v), Manipulate_into_doing (mogeln/cheat.v) und Work (arbeiten/work.v) evoziert, sie werden im KonstruktFrame allerdings durch den Konstruktions-Frame Motion angepasst (Unterabschnitt 4.4.1).

4. Die ,incidental'-Lesart entsteht in einer Teilmenge von Konstrukten, deren Konstrukt-Frames zugleich durch einen lexikalischen Frame und den Konstruktions-Frame konstituiert werden. Voraussetzung dafür ist, dass der Anteil des lexikalischen Frames möglichst gering ist und keine Fusionen zwischen den FE beider Frames (d.h. doppelte semantische Motivierungen eines KtE oder KEE, vgl. Unterabschnitte 6.2.3 und 6.3.3) vorhanden sind. So sind die KtE des KE WEG bzw. 〈WEG〉 sowie die KEE in den Konstrukten in (21) ausschließlich Instanzen von FE des Konstruktions-Frames, nicht des jeweiligen lexikalischen Frames.

Ad Prinzip 1. Ist an einem Konstrukt-Frame allein ein lexikalischer Frame beteiligt, entsteht in vielen Fällen eine neutrale Lesart. Dies zeigt sich am deutlichsten bei dem lexikalischen Frame, der direkt dem Konstruktions-Frame Motion entspricht. Besonders die LE bewegen (move.v) als KtE des KE EREIGNIs lässt keine 
andere Interpretation $\mathrm{zu}$ als eine neutrale Lesart, die nicht auf einen ,manneroder ,means'-Aspekt hin spezifiziert ist, wie die Belege in (22) illustrieren sollen. ${ }^{27}$

(22) a. Aber auch Carvers Figuren [Motion bewegen] sich als Voyeure durch ihre Welt. (Die Zeit, 23.03.2000, Nr. 13)

b. Die augenblickliche Wirtschaftslage [Motion bewege] sich in Richtung Armut, was das Resultat der ausbeuterischen Privatisierung sei. (Archiv der Gegenwart, 2001 [2000])

c. Die von Beduinen geführte Karawane (bis zu 18 Teilnehmer) [Motion bewegt] sich in täglichen Etappen von vier bis sechs Stunden über Dünen und Sandfelder, durch die Wadis und Ebenen, wobei die Kamele als Reit- und Packtiere fungieren. (Die Zeit, 17.02.2000, Nr. 8)

d. Schiller war kein Autor, der von innen kommt, er [Motion bewegte] sich in der Gegenrichtung, von außen nach innen. (Safranski, Rüdiger: Friedrich Schiller, München Wien: Carl Hanser 2004, S. 118)

e. Über der Eingangstür [Motion bewegt] sich die DM im schwerelosen Raum um unseren globalisierten Planeten. (Die Zeit, 17.02.2000, Nr. 8)

f. Ein Drahtseil ist über den Fluß gespannt, mit einer beweglichen Winde ist das Fährboot daran festgetäut, und während die gewaltige Strömung auf das querstehende Ruder drückt, [Motion bewegen] sich Boot und Winde seitlich am Seil entlang von einem Ufer zum andern. (Düffel, John von: Vom Wasser, München: dtv 2006, S. 192)

g. Der Rücken vor ihr, in einem dicken, warmen, grauen Mantel, [Motion bewegte] sich zu einem Bahnsteig, Malka folgte ihm. (Pressler, Mirjam: Malka Mai, Weinheim Basel: Beltz \& Gelberg 2001, S. 246)

27 Goldberg (1995: 205) stuft für die way-Konstruktion Konstrukte mit solchen ,vanilla motion verbs“ als inakzeptabel ein, wobei sie diese Entscheidung mit der in ihnen nicht vorhandenen ,manner'- oder ,means'-Lesart in Verbindung bringt (eine neutrale Lesart wie im FrameNetKonstruktikon erwägt Goldberg nicht) und argumentiert, dass die mangelnde Akzeptabilität dieser Konstrukte an dem nicht vorhandenen Aspekt der ,Schwierigkeit' liegt, der vor allem in Konstrukten mit einer ,means'-Lesart entsteht. Ich komme auf diese Zusammenhänge im Zuge der Diskussion um den Parameter der emergenten Struktur in Abschnitt 5.7 zurück. Der prinzipiellen Möglichkeit der Identität zwischen lexikalischem Frame und Konstruktions-Frame scheint sich Goldberg (1995: 35) - freilich ohne frame-semantisch zu argumentieren - für das Beispiel der Ditransitivkonstruktion indes durchaus bewusst zu sein: „Give, however, is the most prototypical ditransitive verb because its lexical semantics is identical with what is claimed here to be the construction's semantics.“ An anderer Stelle ganz ähnlich: „It is clear that the most common and prototypical case is one in which the verb and the construction do not designate two separate events. Rather the verb designates the same event that the construction designates, or the verb elaborates the constructional meaning.“ (Goldberg 2010: 53). 
Gleiches gilt für die LE begeben (go.v), deren lexikalische Bedeutung ebenfalls Motion zur Grundlage hat. Auch sie lässt nur die Interpretation einer neutralen Lesart zu, wie die Belege in (23) zeigen.

a. Das Quartett [Motion begibt] sich in das Theater Athénée, wo eine leichte Komödie auf dem Programm steht, danach in die Rue Royale, zu einem späten Abendessen ins Restaurant Weber, unweit der Place de la Concorde. (Die Zeit, 13.01.2000, Nr. 3)

b. Sofort [Motion begibt] sich ihr junger Begleiter von RTL an ihre Seite. (Die Zeit, 27.01.2000, Nr. 5)

c. Es lässt sich nicht ganz klären, auf welches Stockwerk die Linke sich neuerdings [Motion begibt]. (Die Zeit, 17.02.2000, Nr. 8)

d. Am 12. Juli [Motion begab] sich KHATAMI nach Weimar, wo er gemeinsam mit RAU ein Denkmal für die Dichter Goethe und Hafis als Symbolfiguren des Dialogs der deutschen und der persischen Kultur enthüllte. (Archiv der Gegenwart, 2001 [2000])

e. Oder aber Sie [Motion begeben] sich unter die Langschläfer, denn in den Halbschlafphasen am späteren Morgen wird auch viel geträumt. (Die Zeit, 20.04.2000, Nr. 17)

f. HASSAN [Motion begab] sich Mitte Oktober in Begleitung einer tausend Mann starken bewaffneten Eskorte von Djibouti aus nach Mogadischu, um seine Macht von dort aus auszuüben. (Archiv der Gegenwart, 2001 [2000])

g. Zu bestimmten Zeiten, wenn die Buschtrommel des HSV ins Volksparkstadion oder die des Ersten FC Kaiserslautern auf den Betzenberg ruft, verlassen die Fans ihre Lehmhütten, [Motion begeben] sich zu den Versammlungsplätzen der Männer, nehmen berauschende Getränke oder Drogen ein, schmücken sich mit den Totems des Stammes und tragen auf ihrer Haut die Farben der Kriegsbemalung auf. (Schwanitz, Dietrich: Männer, Frankfurt a. M.: Eichborn 2001, S. 40)

Auch die lexikalischen Bedeutungen der LE rühren (24) und verschieben (25) (beide move.v) erzeugen eine neutrale Lesart.

(24) a. Stocksteif stand er da, [Motion rührte] sich nicht mehr von der Stelle, starrte nur vor seine Füße. (Funke, Cornelia: Tintenherz, Hamburg: Cecilie Dressler Verlag 2003, S. 241)

b. Vater und Tochter [Motion rührten] sich nicht von ihren Stühlen und schienen doch einander nähergerückt. (Hahn, Ulla: Unscharfe Bilder, München: Deutsche Verlags-Anstalt 2003, S. 275) 
(25) Der Schwerpunkt des Westens [Motion verschob] sich in die USA. (Die Zeit, 02.03.2000, Nr. 10)

Dass bei der Feststellung einer neutralen Lesart das Kriterium der Verteilung lexikalischer Bedeutungen und nicht allein derjenigen lexikalischen Frames angesetzt werden muss, wird bei anderen LE deutlich, die denselben lexikalischen Frame wie diejenigen in den soeben diskutierten Beispielen evozieren. Damit ist der Übergangsbereich zur ,manner'-Lesart erreicht.

Ad Prinzip 2. Während die lexikalischen Bedeutungen von LE wie bewegen, begeben, rühren und verschieben eine neutrale Lesart erzeugen, verhält es sich bei anderen LE, die Moti on evozieren, anders. So verfügen LE wie schlängeln (snake.v) (26), winden (wind.v) (27) und mäandern (meander.v) (28) über einen in der Verbwurzel kodierten ,manner'-Aspekt (im Sinne von Talmy 2007: 72-88), der sich auf die Lesart des Konstrukts auswirkt. Ihre lexikalischen Bedeutungen erzeugen also keine neutrale, sondern eine ,manner'-Lesart.

(26) a. Ich [Motion schlängelte] mich durch die Autos und die Abgase, die rot zwischen den Schlußlichtern dampften, und entdeckte gerade noch rechtzeitig die kräftige Gestalt von John Bird hinter dem erleuchteten Schaufenster. (Franck, Julia: Lagerfeuer, Köln: DuMont Literatur und Kunst Verlag 2003, S. 256)

b. Bald [Motion schlängelten] sich so viele Kabel durch die Wiese und über den Betonboden, daß er alle paar Meter strauchelte. (Glavinic, Thomas: Die Arbeit der Nacht, München Wien: Carl Hanser Verlag 2006, S. 379)

c. Zu beiden Seiten [Motion schlängelten] sich Blumenbeete um schmale Rasenstreifen. (Düffel, John von: Houwelandt, Köln: DuMont Literatur und Kunst Verlag 2004, S. 175)

(27) a. Jonas [Motion wand] sich aus dem Kofferraum nach vorne auf die Rückbank des Autos. (Glavinic, Thomas: Die Arbeit der Nacht, München Wien: Carl Hanser Verlag 2006, S. 346)

b. Ich bekam ihren Arm zu fassen, aber sie [Motion wand] sich aus meinem Griff. (Franck, Julia: Lagerfeuer, Köln: DuMont Literatur und Kunst Verlag 2003, S. 53)

(28) So [Motion mäandern] sich die nackten und verschlungenen Leiber durch die Werbung und über die Titel jener der Werbeästhetik folgenden Magazine. (Die Zeit, 13.01.2000, Nr. 3)

Die ,manner'-Lesart ist, wie bereits angedeutet, definiert als ,, co-occuring activity that is not causally related to the action designated by the construction. “ (Gold- 
berg 1997: 395). Im Falle der in der Verbwurzel kodierten ,manner-Komponente ist die dabei implizierte Distinktion zweier Ereignisse allerdings nicht auf das Vorhandensein zweier unterscheidbarer Frames zurückzuführen. Sind, wie in diesem Fall, lexikalischer Frame und Konstruktions-Frame identisch, hängt es stark von der lexikalischen Bedeutung der LE ab, ob das Konstrukt eine neutrale Lesart oder eine ,manner-Lesart erhält. Dies ist der Kern der oben formulierten Prinzipien 1 und 2: Die Beteiligung nur eines Frames, des lexikalischen Frames, an der Konstitution des Konstrukt-Frames erzeugt nicht zwangsläufig stets eine neutrale Lesart. Die Notwendigkeit zur Unterscheidung zwischen lexikalischem Frame und lexikalischer Bedeutung (Unterabschnitt 4.1.1) zeigt sich hier sehr deutlich: Die Frage nach der Erzeugung einer bestimmten Lesart kann nicht in Bezug auf einen lexikalischen Frame allein beantwortet werden, sondern muss unter Rückgriff auf die auf ihm beruhenden lexikalischen Bedeutungen gestellt werden. ${ }^{28}$

Diese Beobachtung gilt nicht nur für Fälle, in denen der lexikalische Frame dem Konstruktions-Frame entspricht. Auch die Lesart der Konstrukte, deren lexikalischer Frame in einer Frame-zu-Frame-Relation zum Konstruktions-Frame steht, variiert zum Teil innerhalb eines lexikalischen Frames, je nachdem welche lexikalische Bedeutung vorliegt. In den Tabellen 5.2 und 5.3 sind für die reflexive Bewegungskonstruktion und die reflexive Partikelverbkonstruktion getrennt die lexikalischen Bedeutungen (repräsentiert durch die entsprechenden LE) aller belegten lexikalischen Frames in Frame-zu-Frame-Relationen zum KonstruktionsFrame Motion aufgeführt, ${ }^{29}$ einschließlich Motion selbst, geordnet nach der Lesart, die sie in den Konstrukten erzeugen. ${ }^{30}$ Für die reflexive Weg-Konstruktion erübrigt sich eine analoge Aufstellung, da für sie ohnehin nur Motion mit der LE

28 Dass beide Klassen von LE, sowohl diejenigen, die eine neutrale Lesart erzeugen, als auch diejenigen, die eine ,manner'-Lesart erzeugen, unter demselben lexikalischen Frame in FrameNet geführt werden, gibt Anlass zu der Kritik, dass FrameNet, trotz seines grundsätzlichen SplittingAnsatzes, hier zu starkes Lumping betreibt (vgl. zu dieser Kritik auch Perek 2015: 118-119): Zumindest für die Frage nach der Lesart in den Konstrukten der reflexiven Bewegungskonstruktion ist der lexikalische Frame allein somit nicht aussagekräftig. Die Unterscheidung zwischen lexikalischem Frame und lexikalischer Bedeutung muss deshalb in jedem Fall zur Feindifferenzierung getroffen werden.

29 In Abschnitt 5.4 deute ich diese Relationen lexikalischer Frames zum Konstruktions-Frame als Frame-Nähen.

30 Für die Partikelverben, die für die reflexive Partikelverbkonstruktion belegt sind, sind in Tabelle 5.3 i.d.R. nur deren Basisverben aufgenommen. Nur wenn das Partikelverb einer simplizischen englischen LE entspricht (z.B. descend.v für herablassen), ist das Partikelverb als solches aufgenommen. Angaben über die quantitative Verteilung der Konstrukte auf die in den Tabellen 5.2 und 5.3 aufgelisteten lexikalischen Frames sind Gegenstand von Kapitel 6 und deswegen in diesen Tabellen nicht enthalten. 
bahnen in neutraler Lesart belegt ist (vgl. auch die Ergebnisse in Unterabschnitt 7.2.2). Der einzig weitere belegte lexikalische Frame ist Cutt ing mit der LE säbeln (etwa: cut.v) in ,means'-Lesart.

Erkennbar ist, dass zwar einige lexikalische Frames für die reflexive Bewegungskonstruktion und die reflexive Partikelverbkonstruktion belegte lexikalische Bedeutungen subsumieren, die ausschließlich eine neutrale Lesart erzeugen (so Change_direction, Departing, Fleeing, Fluidic_motion, Mass_motion, Motion_directional und Ride_vehicle), viele lexikalische Frames aber sowohl eine neutrale als auch eine ,manner'-Lesart zulassen. Der Frame Body_movement tendiert hierbei stark zur ,manner'-Lesart.

Was bedeuten diese Beobachtungen für die konstruktikographische Erfassung etwa der reflexiven Bewegungskonstruktion hinsichtlich ihrer Lesarten? Wenn die lexikalischen Frames allein keine eindeutigen Präferenzen für die Erzeugung einer bestimmten Lesart zeigen, so reicht eine alleinige Erfassung der lexikalischen Frames, die als Grundlage für lexikalische Bedeutungen dienen, nicht aus. Demnach müssen die Konstrukte, die einem jeden lexikalischen Frame zugeordnet werden können, in einem nächsten Schritt nach den lexikalischen Bedeutungen, denen dieser Frame zugrunde liegt, feindifferenziert werden. Idealerweise müssen dazu die Standardwerte, die in jeder einzelnen lexikalischen Bedeutung für den lexikalischen Frame spezifiziert werden, erfasst werden. Als Heuristik bietet sich allerdings ein Rückgriff auf die unterschiedlichen LE an, wie ich ihn in den Tabellen 5.2 und 5.3 angedeutet habe. Die auf der Basis lexikalischer Bedeutungen getroffene Zuordnung von Lesarten sollte in jedem Fall konstruktikographisch erfasst werden, da nur so deutlich wird, von welchen Faktoren die Erzeugung einer Lesart konkret abhängt (dazu Unterabschnitt 7.2.2).

Ad Prinzip 3. Alle bisher betrachteten Fälle einer neutralen oder auf die lexikalische Bedeutung der LE zurückgehenden ,manner'-Lesart betreffen Konstrukte, deren Konstrukt-Frames durch lexikalische Frames allein konstituiert werden. Nimmt man nun diejenigen Konstrukte hinzu, an deren Konstrukt-Frames sowohl lexikalischer Frame als auch Konstruktions-Frame beteiligt sind, lassen sich die Konstrukte, die zur ,means'-Lesart gezählt werden müssen, ermitteln. Die ,means'-Lesart impliziert, wie Goldberg (1995: 203) für die way-Konstruktion festhält, ,that the path [...] through which the motion takes places is not preestablished, but rather is created by some action of the subject referent." Dieses zusätzlich zu der ,Bewegung، stattfindende Ereignis muss also von jener ,Bewegung' separiert sein - der Grund, warum dafür in erster Linie Konstrukte infrage kommen, deren Konstrukt-Frames durch lexikalischen Frame und Konstruktions- 
Tab. 5.2: Lexikalische Frames und LE in neutraler und ,manner-Lesart für die reflexive Bewegungskonstruktion

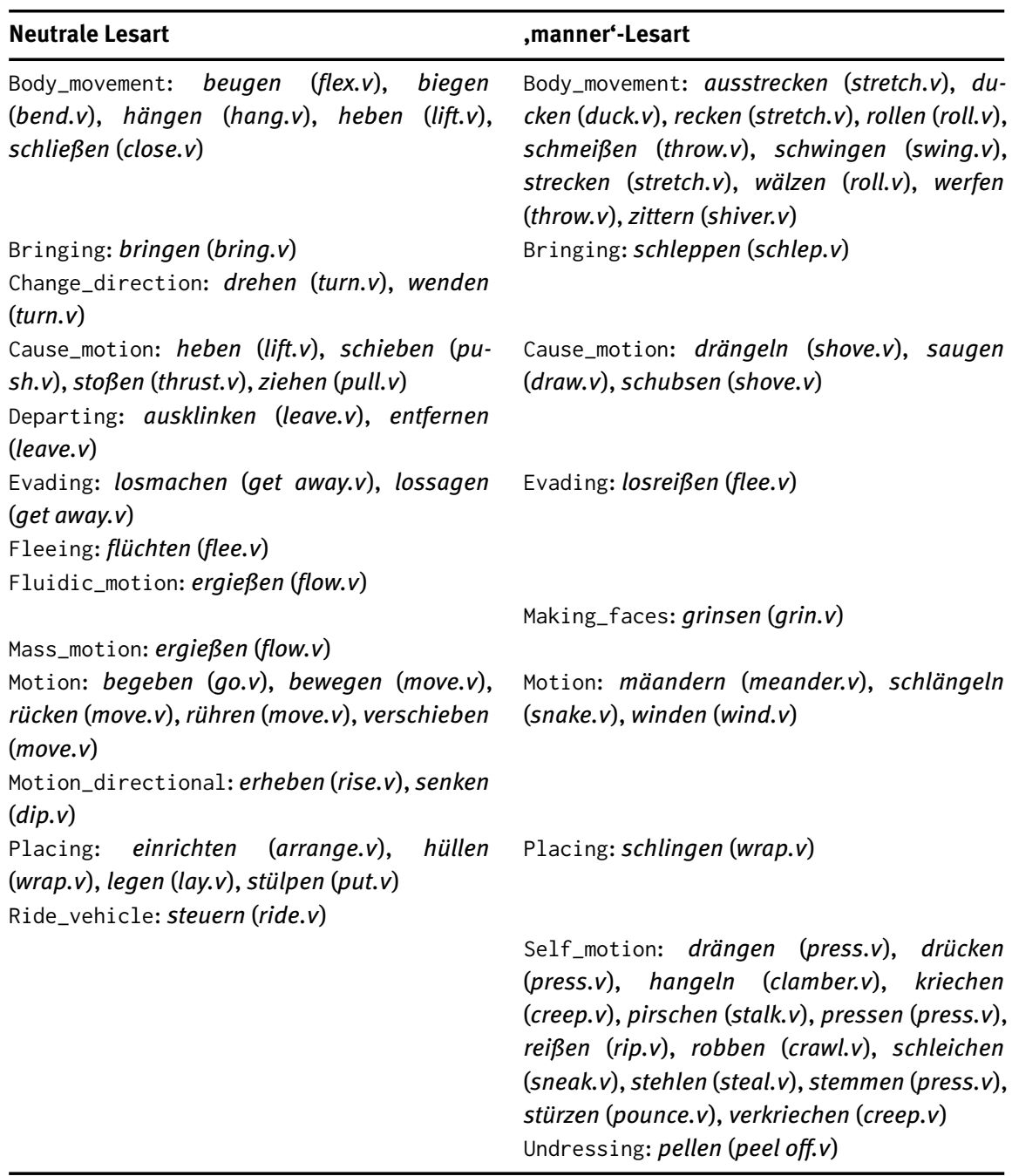

Frame konstituiert werden, in denen der jeweilige lexikalische Frame also durch den Konstruktions-Frame angepasst wird. ${ }^{31}$

31 Inwiefern die ,means'-Lesart dadurch mit dem Aspekt der ,Schwierigkeit‘ verbunden ist, auf dem Goldberg (1995: 203-209) ganz wesentlich ihre Argumentation für die ,means'-Lesart der 
Tab. 5.3: Lexikalische Frames und LE in neutraler und ,manner'-Lesart für die reflexive Partikelverbkonstruktion

\begin{tabular}{|c|c|}
\hline Neutrale Lesart & ,manner'-Lesart \\
\hline $\begin{array}{l}\text { Body_movement: beugen (flex.v), biegen } \\
\text { (bend.v), bücken (bend.v), heben (lift.v) }\end{array}$ & $\begin{array}{l}\text { Body_movement: ausstrecken (stretch.v), du- } \\
\text { cken (duck.v), krümmen (arch.v), recken } \\
\text { (stretch.v), rollen (roll.v), schmeißen (throw.v), } \\
\text { wälzen (roll.v), werfen (throw.v) }\end{array}$ \\
\hline \multirow{3}{*}{$\begin{array}{l}\text { Bringing: bringen (bring.v) } \\
\text { Cause_motion: abstoßen (push.v), heben } \\
\text { (lift.v), schieben (push.v), ziehen (pull.v), } \\
\text { Change_direction: drehen (turn.v), wenden } \\
\text { (turn.v) }\end{array}$} & Bringing: schleppen (schlep.v) \\
\hline & $\begin{array}{l}\text { Cause_motion: drängeln (shove.v), katapultie- } \\
\text { ren (catapult.v) }\end{array}$ \\
\hline & Excreting: rotzen (sneeze.v) \\
\hline \multicolumn{2}{|l|}{ Filling: einhüllen (wrap.v), stauen (jam.v) } \\
\hline \multirow{3}{*}{$\begin{array}{l}\text { Motion: bewegen (move.v) } \\
\text { Motion_directional: herablassen (de- } \\
\text { scend.v), hinablassen (descend.v), senken } \\
\text { (dip.v) }\end{array}$} & $\begin{array}{l}\text { Motion: schlängeln (snake.v), schwingen } \\
\text { (swing.v), winden (wind.v) }\end{array}$ \\
\hline & \\
\hline & Operate_vehicle: rudern (row.v) \\
\hline \multirow[t]{2}{*}{$\begin{array}{l}\text { Placing: legen (lay.v), setzen (put.v), wickeln } \\
\text { (wrap.v) }\end{array}$} & \\
\hline & $\begin{array}{l}\text { Self_motion: drängen (press.v), drücken } \\
\text { (press.v), fuhrwerken (bustle.v), pirschen } \\
\text { (stalk.v), ranken (climb.v), schaukeln } \\
\text { (swing.v), schleichen (sneak.v), schwim- } \\
\text { men (swim.v), stemmen (press.v), stürzen } \\
\text { (pounce.v) }\end{array}$ \\
\hline
\end{tabular}

Als grobes Prinzip lässt sich festhalten, dass die ,means'-Lesart nur dann entstehen kann, wenn lexikalischer Frame und Konstruktions-Frame deutlich voneinander unterschieden sind. ${ }^{32}$ Umgekehrt: Ist für den Konstrukt-Frame der lexikalische Frame allein verantwortlich, kann keine ,means'-Lesart entstehen. Anders als bei den Konstrukten, deren Konstrukt-Frames allein durch einen lexikalischen Frame konstituiert werden, sind die beiden in der ,means'-Lesart vereinten Ereignisse - die ,Bewegung' der durch die KtE von BEWEGENDEs bezeichneten Entität sowie das dafür ursächliche durch das KtE des KE EREIGNIS evozierte Ereig-

way-Konstruktion aufbaut, lasse ich an dieser Stelle offen. Den Aspekt der ,Schwierigkeit‘ rücke ich in Unterabschnitt 5.7.2 in den Fokus der Analyse der reflexiven Bewegungskonstruktion.

32 Was genau,deutlich“ hier bedeutet, erläutere ich ebenso in Abschnitt 5.4 mit dem Begriff der Frame-Nähe. 
nis - mit den zwei Frame-Typen des lexikalischen Frames und des KonstruktionsFrames zu korrelieren. In jedem Fall können unter der ,means'-Lesart vorrangig solche Konstrukte subsumiert werden, deren Konstrukt-Frames aus lexikalischem Frame und Konstruktions-Frame gemeinsam konstituiert werden. ${ }^{33}$

Drei lexikalische Frames sollen dies für die reflexive Bewegungskonstruktion exemplarisch verdeutlichen: In den folgenden Konstrukten mit den lexikalischen Frames Work (29), Ingestion (30) und Mani pulation (31) liegt jeweils eine ,means`Lesart vor.

(29) a. Er [work arbeitete] sich durch ein kompliziertes Kreuzworträtsel, hatte ein Bier aufgemacht und sich auf einen beschaulichen Abend eingestellt, als das Telefon läutete. (Glavinic, Thomas: Die Arbeit der Nacht, München Wien: Carl Hanser Verlag 2006, S. 381)

b. Sie hörte das knarrende Geräusch, als die Scherenblätter aufgingen, spürte das Metall an ihrem Hals und dann [work arbeitete] sich die Schere säbelnd und schabend durch ihren Zopf. (Pressler, Mirjam: Malka Mai, Weinheim Basel: Beltz \& Gelberg 2001, S. 269)

(30) a. Also [Ingestion aßen] sich Kohl und Rühe tapfer durch große Stücke Marzipantorte, unter der Aufsicht von mindestens 15 Kamerateams und unter den kritischen Blicken eines ganzen Schwarms von Reportern, die lauerten und hofften - worauf eigentlich? (Die Zeit, 27.01.2000, Nr. 5)

b. Auch wenn ich als Kind die Inhalte der Angst nicht kapierte, [Ingestion fraß] sich das Gefühl der Angst in den Kopf. (Müller, Herta: Der König verneigt sich und tötet, München: Carl Hanser Verlag 2003, S. 161)

(31) a. Der Ministerpräsident [Manipulation zwängt] sich samt Tross durch die kahlen Betonkavernen, sieht die armen Betten dicht an dicht, dazu die Notmatratze. (Die Zeit, 13.04.2000, Nr. 16)

b. Sie [Manipulation quetschten] sich ebenfalls in den Raum, der nicht viel größer als das Ehebett war, das in ihm stand. (Widmer, Urs: Das Buch des Vaters, Zürich: Diogenes 2004, S. 142)

Konstrukte mit einer ,means'-Lesart sind in besonderer Weise von Koerzionseffekten betroffen, da durch die gemeinsame Konstitution des Konstrukt-Frames

33 Wie Talmy (2007: 80-81) argumentiert, ist aber bereits ein ,manner'-Aspekt kognitiv als zwei voneinander unterschiedene Ereignisse zu konzeptualisieren. Dies dürfte ein Grund dafür sein, warum auch bestimmte Konstrukte, in denen lexikalischer Frame und Konstruktions-Frame deutlich voneinander unterschieden sind, die Interpretation einer ,manner'-Lesart zulassen, worauf ich gleich zurückkomme. 
durch lexikalischen Frame und Konstruktions-Frame Ersterer durch Letzteren in verschieden möglichen Ausprägungen koerziert wird (Abschnitte 5.5 und 7.4).

Ad Prinzip 4. Koerzion spielt auch bei der ,incidental'-Lesart eine wichtige Rolle. Diese Lesart wird von Perek (2018: 69) definiert als „incidental-action sense, in which the action described by the verb merely occurs concomitantly with motion, but is not directly related to it, let alone causes or enables it as in the path-creation [,means', A.W.] interpretation.“ Aus konstruktionssemantischer Sicht stellen Konstrukte mit dieser Lesart eine Teilmenge jener Konstrukte dar, deren KonstruktFrames - wie bei der ,means'-Lesart - durch den Konstruktions-Frame und einen von diesem deutlich unterschiedenen lexikalischen Frame konstituiert sind. Damit die ,incidental'-Lesart entstehen kann, müssen jedoch zwei Voraussetzungen gegeben sein: Das KtE des KE WEG bzw. 〈WEG〉 sowie das KEE müssen ausschließlich Instanzen von FE des Konstruktions-Frames sein und dürfen keinen Anteil des lexikalischen Frames aufweisen. Es handelt sich dabei also um eine FrameAnpassung der Variante c (Unterabschnitt 4.4.1). Bevor ich diese Zusammenhänge in den Abschnitten 6.2 und 6.3 als semantische Motivierung dieser KtE bzw. des KEE diskutiere, ${ }^{34}$ sei für die folgenden Beispiele auf die dortigen Ergebnisse vorgegriffen.

Um die Zusammenhänge zu verdeutlichen, sei in den folgenden Beispielen die Annotation der KtE des KE WEG sowie des KEE auf die FE, durch die sie semantisch motiviert werden, dargestellt. ${ }^{35}$ Als ein erster lexikalischer Frame seien in (32) Konstrukte mit Hostile_encounter zitiert.

a. David [Hostile_encounter kämpfte] [sich ${ }^{\text {THEME}] ~[d u r c h ~ d a s ~ u ̈ b e r f u ̈ l l t e ~ L o-~}$ $\left.k{ }^{\mathrm{PATH}}\right]$ und reduzierte seinen Wortschatz auf: Moment, gleich, Sekunde, bin schon da und sofort. (Suter, Martin: Lila, Lila, Zürich: Diogenes 2004, S. 52)

b. Ich [Hostile_encounter kämpfte] [mich THEME] [durch dieses Unterholz $\left.{ }^{\mathrm{PATH}}\right]$, hielt mich an den Stämmen, Asten und Wurzeln fest, weil ich hinauf zum Gipfel wollte, der wie jeden Tag in den Wolken lag; wie dick die Schicht war, ließ sich nicht sagen - vielleicht schien oben die Sonne. (Schrott, Raoul: Tristan da Cunha oder die Hälfte der Erde; Hanser Verlag 2003, S. 161)

34 Zum Begriff der semantischen Motivierung vgl. speziell Unterabschnitt 6.1.2.

35 Die tiefgestellte Annotation vor dem jeweiligen Element zeigt die FE des lexikalischen Frames, während die hochgestellte Annotation nach dem Element FE des Konstruktions-Frames zeigt. Da es sich bei Letzterem immer um Motion handelt, ist sein Name nicht zusätzlich in der Annotation dargestellt. 
Ein weiterer lexikalischer Frame, der für die ,incidental'-Lesart infrage kommt, ist Daring. Einige Konstrukte hierfür samt der semantischen Motivierung der KtE des KE WEG und des KEE sind in (33) dargestellt.
a. Weil die Probleme offenbar noch nicht reichen, [Daring wagt] [sich THEME] Volkswagen auch noch [in die Luxusregionen GOAL]. (Die Zeit, 30.03.2000, Nr. 14)
b. Kopp [Daring traute] [sich ${ }^{\text {THEME }}$ ] nicht mehr [aus seinem Zimmer SouRCE]. (Koneffke, Jan: Paul Schatz im Uhrenkasten, Köln: DuMont Buchver- lag 2000, S. 74)

Ein anderes Beispiel ist der lexikalische Frame Make_noise, bei dem für die reflexive Bewegungskonstruktion das KtE des KE WEG und das KEE ebenfalls allein durch FE des Konstruktions-Frames motiviert werden. Zwei Konstrukte dafür sind in (34) zusammengestellt.

(34) a. Unter www.skispringen.de [Make_noise klickte] [sich THEME] der Surfer [durch Ergebnistabellen, Springer-Biografien, -Homestorys und -Interviews ${ }^{\text {РАTH}], ~ e r ~ k o n n t e ~ s i c h ~ d i e ~ b e s t e n ~ S p r u ̈ n g e ~ n o c h m a l s ~ a u f ~}$ Video ansehen, mit Günther Jauch und anderen chatten oder am Gewinnspiel teilnehmen. (Die Zeit, 13.01.2000, Nr. 3)

b. Sie [Make_noise klicken] [sich ${ }^{\mathrm{THEME}}$ ] [durch Portale ${ }^{\mathrm{PATH}}$ ], orientieren sich an Sitemaps, immer auf der Suche nach der besten Adresse. (Die Zeit, 03.02.2000, Nr. 6)

Als letztes Beispiel für die ,incidental'-Lesart soll der lexikalische Frame Dead_or_ alive dienen. Auch bei einem Konstrukt-Frame mit ihm sind die beiden einschlägigen Strukturelemente ausschließlich durch den Konstruktions-Frame motiviert, weshalb eine, incidental'-Interpretation plausibel erscheint. Ein für die reflexive Bewegungskonstruktion belegtes Konstrukt ist in (35) zu sehen.

(35) Man [Dead_or_alive lebte] [sich ${ }^{\text {THEME}] ~[a u s ~ d e r ~ h e r a u s g e r i s s e n e n ~ Z e i t, ~ i n ~ d e r ~}$ man saß SOURCE], zurück in die Erinnerung ans Zuhause von damals und voraus in die Hoffnung, bald heimzukehren. (Müller, Herta: Der König verneigt sich und tötet, München: Carl Hanser Verlag 2003, S. 42)

Durch die Betrachtung der semantischen Motivierung der Strukturelemente relevanter Konstrukte, also die Konstitution entsprechender Konstrukt-Frames, wird nun deutlich, warum Koerzion für die ,incidental'-Lesart eine so große Rolle spielt. Werden zwei der vier Strukturelemente eines Konstrukts durch den KonstruktionsFrame motiviert, ist der Anteil des lexikalischen Frames sehr gering. Anders gewendet: die Koerzion durch den Konstruktions-Frame ist hoch. Dieser Fall ist einer 
von mehreren, die das Koerzionspotenzial einer Konstruktion (Abschnitt 5.5) auszeichnen. Wie ich in Unterabschnitt 7.4.1 zeigen werde, ist die in den Konstrukten in (32)-(35) zu beobachtende Koerzion die stärkstmögliche, die ein KonstruktionsFrame zu leisten in der Lage ist. Dadurch, dass der lexikalische Frame derart stark durch den Konstruktions-Frame koerziert wird, geht eine mögliche Verbindung beider Frames wie im Falle der ,means'-Lesart tendenziell verloren, was in Konsequenz zu der ,incidental'-Lesart führt, die eben keine solche Verbindung impliziert.

Ein Vergleich zwischen zwei Konstrukten mit demselben lexikalischen Frame, aber unterschiedlichen semantischen Motivierungen der KtE und des KEE soll dies abschließend demonstrieren. Hierzu sei zusätzlich zur reflexiven Bewegungskonstruktion die reflexive Partikelverbkonstruktion herangezogen. Die beiden Konstrukte in (36) und (37) zeigen für die reflexive Bewegungskonstruktion in (36) und die reflexive Partikelverbkonstruktion in (37) jeweils einen KonstruktFrame mit dem lexikalischen Frame Impact. Im Konstrukt in (36) wird das KtE des KE WEG durch eine Fusion des FE Impact.IMPACTEE des lexikalischen Frames mit dem FE Motion.PATH des Konstruktions-Frames semantisch motiviert. Darüber hinaus ist das KEE allein durch den Konstruktions-Frame und das FE Motion.THEME motiviert. Das Ergebnis ist eine ,means'-Lesart des Konstrukts.

(36) Der Vater [Impact schlug] [sich ThEmE] [Impactee durch die Haus- und Gartentür, die danach, und für Jahre, schräg in den Angeln hing ${ }^{\mathrm{PATH}}$ ]. (Widmer, Urs: Das Buch des Vaters, Zürich: Diogenes 2004, S. 153)

In dem Konstrukt in (37) hingegen ist eine solche Fusion nicht gegeben: Das KtE des KE $\langle$ WEG $\rangle$ wird allein durch das FE Motion.GoAL des Konstruktions-Frames motiviert. Da auch das KEE nach wie vor durch den Konstruktions-Frame und das FE Motion.THEME motiviert ist, bleibt in diesen beiden Strukturelementen des Konstrukts kein Anteil des lexikalischen Frames übrig, die Koerzion ist somit höher als in (36). Das Ergebnis ist eine, incidental'-Lesart.

„Repetieren: morgen, in einer Woche, in einem Monat“ heißt Staubs Rezept, damit [sich ${ }^{\text {THEME}] ~ d a s ~ R e i n g e h a ̈ m m e r t e ~[b i s ~ i n s ~ L a n g z e i t g e d a ̈ c h t n i s ~}$ GoAL] durch[Impact schlägt]. (Die Zeit, 16.03.2000, Nr. 12)

Als ein zweites Beispiel für einen lexikalischen Frame, der sowohl eine ,means'als auch eine ,incidental '-Lesart hervorbringen kann, soll Ingestion dienen, der in die Konstrukt-Frames der Konstrukte der reflexiven Bewegungskonstruktion in (38) und (39) eingeht. In (38) wird das KtE des KE WEG erneut durch eine Fusion von FE aus lexikalischem Frame (Ingestion.INGESTIBLES) und Konstruktions- 
Frame (Motion.PATH) motiviert, während das KEE allein durch Mot ion.THEME motiviert wird. Ergebnis ist eine ,means'-Lesart.

(38) Also [Ingestion aßen] [sich THeme] Kohl und Rühe tapfer [Ingestibles durch große Stücke Marzipantorte ${ }^{\mathrm{PATH}}$ ], unter der Aufsicht von mindestens 15 Kamerateams und unter den kritischen Blicken eines ganzen Schwarms von Reportern, die lauerten und hofften - worauf eigentlich? (Die Zeit, 27.01.2000, Nr. 5)

In (39) wiederum fehlt diese Fusion, das KtE des KE WEG ist allein durch das FE Motion.GoAL motiviert, während die Motivierung des KEE unverändert bleibt. Auch in diesem Konstrukt ist die Koerzion somit höher als in (38), wodurch eine ,incidental'-Lesart entsteht.

(39) Es war allen Anwesenden nachzufühlen; von unschuldigem Frohsinn war nichts zu spüren, die Abende zogen sich dahin, der Steuereintreiber und der Chirurg entschuldigten sich schon früh, um nicht wieder in den Genuß von Gratiskonsultationen zu kommen, der Priester gab seine zwei Witze zum besten, und der Rest lästerte über das Essen und [Ingestion soff] [sich THEME] [unter den Tisch ${ }^{\text {GoAL}}$ ], weil selbst die Konjunktur als Thema längst ausgeschöpft war. (Schrott, Raoul: Tristan da Cunha oder die Hälfte der Erde; Hanser Verlag 2003, S. 196)

Die beiden Konstrukte in (38) und (39) sind zugleich Belege dafür, dass eine pauschale Zuordnung bestimmter Verbklassen zu diesen Lesarten, wie sie Perek (2018: 86-87) etwa für Verben der ,Nahrungsaufnahme‘ (die gewissermaßen Ingestion evozieren) festhält, nicht möglich ist (dazu bereits Unterabschnitt 3.1.3). Auch diese Verbklassen bzw. lexikalischen Frames können in sich wiederum in unterschiedliche Lesarten differenziert sein, je nachdem, wie die semantische Motivierung der einzelnen Strukturelemente des Konstrukts beschaffen ist. In diesem Sinne kritisiert auch Fanego (2019: 693) die Klassifikation Pereks als zu starkes Lumping von eigentlich der ,means'-Lesart zugehörigen Verben und solchen, die tatsächlich eine, incidental'-Lesart bewirken.

Überschneidungen zwischen ,means'- und, incidental'-Lesart, wie sie in (36) und (37) sowie in (38) und (39) zu sehen sind, sind allerdings eher selten belegt. In der Regel ist die einfache Motivierung des KtE des KE WEG bzW. 〈WEG〉 und des KEE durch den Konstruktions-Frame charakteristisch für bestimmte lexikalische Frames. Abschließend seien daher in den Tabellen 5.4 und 5.5 für die reflexive Bewegungskonstruktion und die reflexive Partikelverbkonstruktion die lexikalischen Frames, für die eine ,means'-Lesart belegt ist, denjenigen, für die ei- 
ne ,incidental'-Lesart belegt ist, gegenübergestellt. ${ }^{36}$ Wie bereits für den Vergleich von neutraler und ,manner'-Lesart (Tabellen 5.4 und 5.5) erübrigt sich für die reflexive Weg-Konstruktion eine entsprechende Aufstellung, da für sie mit Cutting nur ein einziger lexikalischer Frame ohne Frame-zu-Frame-Relation zu Motion belegt ist, der eine ,means'-Lesart erzeugt.

Wenngleich zwischen ,means'- und, incidental'-Lesart nur wenige Überschneidungen hinsichtlich der lexikalischen Frames auftreten, deutet die grundsätzliche Möglichkeit zu solchen Überschneidungen darauf hin, dass über die Verteilung lexikalischer Frames und ihre Zuordnung zu den unterschiedlichen Lesarten hinaus weitere Prinzipien existieren müssen, nach denen sich die Entstehung der vier Lesarten richtet.

\subsubsection{Weiterführende Prinzipien zur Differenzierung der Lesarten}

Die in Unterabschnitt 5.2.3 diskutierten vier Prinzipien zur Differenzierung der vier Lesarten sind nicht als feste Regeln zu verstehen, sondern lediglich als Tendenzen bei der Verteilung der einzelnen Lesarten. Darüber hinaus existieren Fälle, die durch das Raster dieser vier Prinzipien fallen. Diese möchte ich nun als Abschluss der Diskussion des semantischen Parameters der konstruktionellen Polysemie in den Blick nehmen.

Wirft man einen Blick auf die ,means'-Lesart und deren Konstitution durch Konstrukte, deren Konstrukt-Frames durch lexikalischen Frame und Konstruktions-Frame gleichermaßen konstituiert werden, so fällt auf, dass keineswegs alle Konstrukte, die diese Voraussetzung erfüllen, auch tatsächlich eine ,means“Lesart erhalten. Ebenso möglich ist die Entstehung einer neutralen oder der ,manner-Lesart. Die Verteilung richtet sich hier deutlicher nach dem lexikalischen Frame als dies bei der ,manner'-Lesart in Konstrukten mit alleinigem lexikalischem Frame der Fall ist. So entsteht mit dem lexikalischen Frame Cause_to_ experience eine ,manner'-Lesart, wie die Konstrukte in (40) zeigen.

(40) a. Die Emanzipationswaisen werden $\mathrm{zu}$ egoistischen Monstern und [Cause_to_experience quälen] sich mit begrenzter Libido durch bretonische Swinger-Clubs. (Die Zeit, 16.03.2000, Nr. 12)

36 Angaben über die quantitative Verteilung der Konstrukte auf die in den Tabellen 5.4 und 5.5 sowie 5.6 und 5.7 (Unterabschnitt 5.2.4) aufgelisteten lexikalischen Frames sind - wie schon für die lexikalischen Frames in den Tabellen 5.2 und 5.3 - Gegenstand von Kapitel 6 und deswegen in diesen Tabellen nicht aufgeführt. 
Tab. 5.4: Lexikalische Frames der reflexiven Bewegungskonstruktion ohne Frame-zuFrame-Relation zu Motion nach ,means'- und ,incidental'-Lesart

\begin{tabular}{|c|c|}
\hline ,means'-Lesart & ,incidental'-Lesart \\
\hline $\begin{array}{l}\text { Assistance } \\
\text { Attaching } \\
\text { Burying } \\
\text { Cause_harm } \\
\text { Cause_to_amalga- } \\
\text { mate }\end{array}$ & $\begin{array}{l}\text { Chatting } \\
\text { Cause_bodily_ex- } \\
\text { perience }\end{array}$ \\
\hline $\begin{array}{l}\text { Cause_to_experi- } \\
\text { ence }\end{array}$ & $\begin{array}{l}\text { Daring } \\
\text { Dead_or_alive }\end{array}$ \\
\hline $\begin{array}{l}\text { Desiring } \\
\text { Emptying } \\
\text { Filling } \\
\text { Grinding }\end{array}$ & \\
\hline Hostile_encounter & $\begin{array}{l}\text { Hostile_encounter } \\
\text { Hunting }\end{array}$ \\
\hline $\begin{array}{l}\text { Impact } \\
\text { Ingestion }\end{array}$ & $\begin{array}{l}\text { Impact } \\
\text { Ingestion } \\
\text { Make_noise }\end{array}$ \\
\hline $\begin{array}{l}\text { Manipulate_in- } \\
\text { to_doing }\end{array}$ & \\
\hline Manipulation & $\begin{array}{l}\text { Progression } \\
\text { Prevarication } \\
\text { Reshaping }\end{array}$ \\
\hline $\begin{array}{l}\text { Rescuing } \\
\text { Scouring } \\
\text { Shopping } \\
\text { Smuggling }\end{array}$ & \\
\hline Work & Work \\
\hline
\end{tabular}

Tab. 5.5: Lexikalische Frames der reflexiven Partikelverbkonstruktion ohne Frame-zuFrame-Relation zu Motion nach ,means'- und ,incidental'-Lesart

\begin{tabular}{l}
,means'-Lesart \\
\hline Assistance \\
Attaching \\
Attack \\
Becoming_aware \\
Board_vehicle \\
Burying \\
Cause_to_amalga- \\
mate \\
Cause_to_experi- \\
ence \\
Change_of_phase
\end{tabular}

Chatting

Cogitation

Daring

Desiring

Emptying

Filling

Fire_burning

Grinding

Hostile_encounter Hostile_encounter

Impact

Impact

Ingestion

Ingestion

Make_noise

Manipulate_in-

to_doing

Manipulation

Participation

Perception_experience

Processing_materials

$\begin{array}{ll}\text { Questioning } & \\ \text { Renting } & \\ \text { Rescuing } & \\ \text { Seeking } & \\ \text { Shopping } & \text { Temperature } \\ \text { Work } & \text { Work }\end{array}$ 
b. Sie [Cause_to_experience quält] sich durch die Tage. (Dölling, Beate: Hör auf zu trommeln, Herz, Weinheim: Beltz \& Gelberg 2003, S. 126)

c. „Ich sitze hier von morgens bis abends am Schreibtisch und [cause_to_ experience quäle] mich durch eine Akte nach der anderen. (Düffel, John von: Houwelandt, Köln: DuMont Literatur und Kunst Verlag 2004, S. 137)

d. Sie [Cause_to_experience quälten] sich vielleicht auch aus Tischen, wenn man voller Zweifel dagegen stieß? (Kuckart, Judith: Lenas Liebe, Köln: DuMont Literatur und Kunst Verlag 2002, S. 123)

e. Vom sonnigen unteren Centovalli aus steigt man über fette Feuersalamander durch Esskastanienwälder bergan, schwitzt, dampft, springt nackt in einen Bergbach, [cause_to_experience quält] sich über verrutschte Pfade an Ziegenvolk vorbei ins Baumlose, zieht einen Pullover an, wirft den ersten Schneeball, und bei 1000 Metern über null versinkt man bis zur Hüfte im Schnee. (Die Zeit, 30.03.2000, Nr. 14)

Unter den Konstrukten, deren Konstrukt-Frames durch lexikalischen Frame und Konstruktions-Frame zugleich zustande kommen, finden sich allerdings auch solche, die eine neutrale Lesart zulassen. Ein Beispiel dafür ist der lexikalische Frame Cause_to_amalgamate in den Belegen in (41).

(41) a. Es war verteufelt, Herr Joseph Schatz [Cause_to_amalgamate mischte] sich in seine Aufmerksamkeit. (Koneffke, Jan: Paul Schatz im Uhrenkasten, Köln: DuMont Buchverlag 2000, S. 221)

b. Er betrachtete mich mit einem Blick, der Einverständnis forderte und in den sich schon die Skepsis [Cause_to_amalgamate mischte], ob er mich, wie viele andere, nicht bald aufgeben müßte. (Die Zeit, 20.04.2000, Nr. 17)

c. Lichtgarben und Wolkenschatten [Cause_to_amalgamate mengen] sich in den Strom, der in seiner Meeresgrüne schäumend aufblitzt. (Düffel, John von: Vom Wasser, München: dtv 2006, S. 69)

d. Selbst Magne Furuholmen von der leicht verdaulichen Popband Aha [Cause_to_amalgamate mischt] sich unter die Runde der exzentrischen Glasperlenspieler, die an ihrer Version von „arctic ambient“ weben. (Die Zeit, 20.04.2000, Nr. 17)

e. Sie [Cause_to_amalgamate mischen] sich unter die Bevölkerung, die den Eroberern ein freudiges Willkommen bereitet. (Die Zeit, 27.04.2000, Nr. 18)

Insgesamt sind die lexikalischen Frames, die eine neutrale Lesart zulassen, allerdings unter den Konstrukten, deren Konstrukt-Frame aus lexikalischem Fra- 
Tab. 5.6: Lexikalische Frames der reflexiven Bewegungskonstruktion ohne Frame-zu-FrameRelation zu Motion nach neutraler und ,manner'-Lesart

\begin{tabular}{ll}
\hline Neutrale Lesart & ,manner'-Lesart \\
\hline $\begin{array}{l}\text { Attaching } \\
\text { Cause_to_amalgamate }\end{array}$ & $\begin{array}{l}\text { Cause_to_experience } \\
\text { Cause_to_move_in_place }\end{array}$ \\
Cause_to_move_in_place \\
Giving & Filling \\
& $\begin{array}{l}\text { Manipulation } \\
\text { Others_situation_as_stimulus } \\
\text { Perception_experience } \\
\end{array}$ \\
& Seeking \\
\hline
\end{tabular}

me und Konstruktions-Frame zugleich konstituiert wird, in der Minderheit. Darüber hinaus bleibt das Kriterium für die ,incidental'-Lesart, dass das KtE des KE WEG bzw. 〈WEG) und das KEE nur durch den Konstruktions-Frame motiviert sein müssen, erhalten. Konstrukte, die dieses Kriterium erfüllen, erhalten stets die ,incidental'-Lesart.

Mögliche Überschneidungen zwischen den Prinzipien der Differenzierung der vier Lesarten betreffen somit allein die neutrale sowie die ,manner- und ,means'-Lesart. Ein Vergleich der Auflistungen in den Tabellen 5.6 und 5.7 mit denjenigen in den Tabellen 5.4 und 5.5 in Unterabschnitt 5.2.3 offenbart, dass einige der für die drei untersuchten Konstruktionen belegten lexikalischen Frames im Hinblick auf die Lesart, die sie erzeugen, mehrdeutig sind: Es sind für die reflexive Bewegungskonstruktion und die reflexive Partikelverbkonstruktion lexikalische Frames ohne Frame-zu-Frame-Relation zu Motion belegt, die eine neutrale oder eine ,manner'-Lesart erzeugen und die sich teils mit denjenigen lexikalischen Frames, die eine ,means'- oder, incidental'-Lesart erzeugen, überschneiden. Hier muss also erneut eine Feindifferenzierung in die unterschiedlichen lexikalischen Bedeutungen, denen ein lexikalischer Frame zugrunde liegt, erfolgen.

Anhand des lexikalischen Frames Cause_to_move_in_place sei eine weitere Besonderheit diskutiert. Dieser Frame steht nicht in einer Frame-zu-FrameRelation zu Motion und kann sowohl eine neutrale als auch eine ,manner-Lesart erzeugen. Anders als bei lexikalischen Frames wie Cause_to_experience oder Cause_to_amalgamate, die wie in der Annotation von (40) und (41) zu sehen, im Konstrukt-Frame durch den Konstruktions-Frame angepasst werden, muss dies bei Cause_to_move_in_place nicht der Fall sein. Cause_to_move_in_place kann eine neutrale oder eine ,manner'-Lesart erzeugen, wenn sowohl das KtE des KE 
Tab. 5.7: Lexikalische Frames der reflexiven Partikelverbkonstruktion ohne Frame-zu-FrameRelation zu Motion nach neutraler und, manner'-Lesart

\begin{tabular}{ll}
\hline Neutrale Lesart & ,manner-Lesart \\
\hline Cause_to_amalgamate & Attaching \\
Cause_to_move_in_place & Cause_to_experience \\
& $\begin{array}{l}\text { Change_posture } \\
\text { Experiencer_focus }\end{array}$ \\
Filling & $\begin{array}{l}\text { Manipulation } \\
\text { Seeking }\end{array}$ \\
\hline
\end{tabular}

WeG bzw. 〈WEG〉 als auch das KEE durch seine FE motiviert sind, der KonstruktFrame also keine Anteile des Konstruktions-Frames enthält. Die Belege in (42) zeigen Konstrukte mit der LE drehen (turn.v), die eine neutrale Lesart besitzen.

(42) a. Ich [cause_to_move_in_place drehte] [THEME mich] wieder [BoDyPART_OF_AGEnT auf den Bauch]. (Goosen, Frank: Liegen lernen, Frankfurt am Main: Eichborn AG 2000, S. 93)

b. Ich [Cause_to_move_in_place drehe] [THeme mich] [BoDypart_of_AGent auf die Seite], Sandra legt sich hinter mich und streichelt mir den Rücken. (Genazino, Wilhelm: Die Liebesblödigkeit, München, Wien: Carl Hanser Verlag 2005, S. 15)

c. Der Schläfer [Cause_to_move_in_place drehte] [THEME $\operatorname{sich}$ ] [BodYPART_OF_AGENT auf die andere Seite]. (Glavinic, Thomas: Die Arbeit der Nacht, München Wien: Carl Hanser Verlag 2006, S. 139)

Ob mit Cause_to_move_in_place als lexikalischem Frame eine neutrale Lesart wie in den Konstrukten in (42) oder eine ,manner'-Lesart entsteht, hängt nun wiederum von der lexikalischen Bedeutung der LE ab, die ihn evoziert. So legen die lexikalischen Bedeutungen von LE wie wiegen (rock.v) oder wellen (wave.v) eine ,manner-Lesart nahe, etwa in (43). Dort ist allerdings zu erkennen, dass der Konstrukt-Frame nicht nur aus FE von Cause_to_move_in_place besteht, sondern nun einen Anteil des Konstruktions-Frames Motion besitzt.

(43) a. Sie [cause_to_move_in_place wiegte] [THEME $\operatorname{sich}$ ] [gegen ihn ${ }^{\text {GoAL}}$. (Hettche, Thomas: Der Fall Arbogast, Köln: DuMont Buchverlag, 2001, S. 14)

b. Als sie den Finger auf eine blaue Blüte schob, färbte sich ihr Fingernagel bläulich und grüne Streifen [Cause_to_move_in_place wellten] [TнемE 
sich] [über ihren Handrücken ${ }^{\mathrm{PATH}}$ ]. (Pressler, Mirjam: Malka Mai, Weinheim Basel: Beltz \& Gelberg 2001, S. 5)

Der Fall, dass ein lexikalischer Frame, der nicht in einer Frame-zu-Frame-Relation zum Konstruktions-Frame steht, dennoch in der Lage ist, einen Konstrukt-Frame vollständig ohne Anteil des Konstruktions-Frames zu konstituieren, wie es für Cause_to_move_in_place in den Konstrukten in (42) der Fall ist, gibt Anlass zu einer weiterführenden Diskussion, auf die ich in Unterabschnitt 6.4.3 zurückkomme. Die in diesem Unterabschnitt diskutierten Beispiele zeigen allerdings bereits, dass sich die Verteilung insbesondere der neutralen, der ,manner'- und der ,means'-Lesart zunächst nach den lexikalischen Frames, aus denen die KonstruktFrames konstituiert sind, richten muss und anschließend nach den lexikalischen Bedeutungen, die auf diesen Frames beruhen, feindifferenziert werden kann. Diese Reihenfolge erscheint als ein grundlegendes Prinzip der Differenzierung von Lesarten und liegt gewissermaßen quer zu den in Unterabschnitt 5.2.3 formulierten Prinzipien, die die Lesarten im Einzelnen betreffen.

Wird der Konstrukt-Frame aus lexikalischem Frame und KonstruktionsFrame zugleich konstituiert, lässt sich anhand des jeweiligen lexikalischen Frames zumindest eine Tendenz für die Entscheidung zwischen ,manner- und ,means'-Lesart treffen. Konstrukte, deren Konstrukt-Frames allein ein lexikalischer Frame zugrunde liegt, müssen darüber hinaus hinsichtlich der lexikalischen Bedeutungen der LE, die das KE EREIGNIS instanziieren, differenziert werden. Wird der Konstrukt-Frame von lexikalischem Frame und KonstruktionsFrame gemeinsam konstituiert, ist eine solche Differenzierung in vielen Fällen ebenso hilfreich. Diese Beobachtungen helfen dabei, die Lesarten der drei Konstruktionen zu unterscheiden und jeder Lesart einschlägige Konstrukte zuzuordnen. Die Ergebnisse dieser Analyse müssen freilich konstruktikographisch ebenso dokumentiert und generalisiert werden wie die Lesarten selbst. Die Betrachtung der lexikalischen Frames und insbesondere der lexikalischen Bedeutungen hilft dabei, den Parameter der konstruktionellen Polysemie in einer konstruktikographischen Analyse zu verarbeiten. Ich komme in Unterabschnitt 7.2.2 darauf zurück.

Die semantischen Nuancen, in denen sich die Lesarten der drei Konstruktionen widerspiegeln, sind Motivation dafür, für diese Art konstruktioneller Polysemie einen Lumping-Ansatz einem Splitting-Ansatz vorzuziehen. Die auf der Konstitution von Konstrukt-Frames und Konstruktbedeutungen, insbesondere aber auf der Unterscheidung zwischen lexikalischen Frames und lexikalischen Bedeutungen beruhende Differenzierung gibt kaum Anlass dazu, für die entstehenden semantischen Nuancen unterschiedliche Konstruktionen und damit Konstruktionseinträge anzunehmen. Aus diesem Grund verfolge ich für die drei untersuch- 
ten Konstruktionen keinen Splitting-Ansatz, sondern einen Lumping-Ansatz, der sich insbesondere darin äußert, dass es für jede Konstruktion lediglich einen einzelnen, umfassenden Konstruktionseintrag geben muss.

Was aus der Analyse konstruktioneller Polysemie darüber hinaus festzuhalten ist, ist die Relevanz lexikalischer Frames und lexikalischer Bedeutungen, die ich für diesen Parameter mehrfach betont habe. Im Eingang dieses Kapitels ist in Abbildung 5.1 deshalb eine Verbindung zwischen dem Parameter der konstruktionellen Polysemie und einem, der sich unter anderem ebenfalls auf lexikalische Frames bezieht, dargestellt: Beschränkungen und Präferenzen. Es ist unschwer zu erkennen, dass sich die Verteilung der Lesarten einer Konstruktion wesentlich nach ihren Präferenzen für lexikalische Frames richtet.

\subsection{Beschränkungen und Präferenzen}

Lexikalische Frames und lexikalische Bedeutungen spielen, dies hat sich zuletzt in Abschnitt 5.2 gezeigt, in ihrer Interaktion insbesondere mit dem KonstruktionsFrame eine entscheidende Rolle. Wie ich in Kapitel 4 bereits angesprochen habe und in Kapitel 6 ausführlicher empirisch zeigen werde, sind sie gegenüber dem Konstruktions-Frame als vorrangig zu betrachten. Somit verwundert es nicht, dass es einen Parameter geben muss, in dem diese Vorrangstellung lexikalischer Frames zum Ausdruck kommt, sind sie es doch, die unter anderem darüber entscheiden, ob ein Konstruktions-Frame überhaupt an der Konstitution eines Konstrukt-Frames beteiligt ist (vgl. Abschnitt 5.4). Aus diesem Grund gehören die Präferenzen für lexikalische Frames, die eine Konstruktion aufweist, zu einem Parameter, der sich unmittelbar auf die die Rolle des Konstruktions-Frames bei der Konstitution von Konstrukt-Frames und seine Verteilung über die Konstrukte einer Konstruktion hinweg auswirkt.

Präferenzen für lexikalische Frames sind zu unterscheiden von Beschränkungen, etwa eines einzelnen Strukturelements hinsichtlich formaler Kriterien seiner Instanziierung. Ich möchte deshalb in Unterabschnitt 5.3.1 zunächst dafür argumentieren, aus einer gebrauchsbasierten Perspektive einen Unterschied zwischen Beschränkungen und Präferenzen zu ziehen und diese Unterscheidung theoretisch begründen. In Unterabschnitt 5.3.2 zeige ich dann einige formale Beschränkungen auf, die die Strukturelemente der drei untersuchten Konstruktionen aufweisen und die in eine konstruktikographische Beschreibung Eingang finden müssen, weshalb sie neben Präferenzen für lexikalische Frames Teil dieses Parameters sind. In Unterabschnitt 5.3.3 zeige ich, dass solche Präferenzen für lexikalische Frames vielfältig mit anderen semantischen Parametern von Konstruktionen in Verbindung stehen, weshalb ich sie als übergeordneten Parameter 
bezeichne. Insgesamt gehe ich in diesem Abschnitt überwiegend auf die theoretische und allgemein konstruktikographische Relevanz dieses Parameters ein. Auf empirische Ergebnisse, in denen insbesondere Präferenzen für lexikalische Frames eine Rolle spielen, komme ich in Kaptitel 6 sowie in den Unterabschnitten 7.1.3 und 8.5.1 zurück.

\title{
5.3.1 Präferenzen vs. Beschränkungen
}

Die Beschreibung von Beschränkungen (constraints) einer Konstruktion gehört von Anbeginn zu den Hauptaufgaben der Konstruktionsgrammatik, wie schon Goldberg (1995) berichtet:

\begin{abstract}
Construction Grammarians also share an interest in accounting for the conditions under which a given construction can be used felicitously, since this is taken to be part of speakers'competence or knowledge of language; from this interest stems the conviction that subtle semantic and pragmatic factors are crucial to understanding the constraints on grammatical constructions. (Goldberg 1995: 6)
\end{abstract}

Hilpert (2019: 18-20) erachtet Beschränkungen gar als konstitutiv für zahlreiche Konstruktionen, da sie als Diagnostikum zu deren Identifizierung herangezogen werden können. Auch aus konstruktikographischer Sicht sind Beschränkungen von Relevanz, da sie sich, wie Borin et al. (2012) argumentieren, aus gebrauchsbasierter Evidenz ergeben:

The linguistic annotations are a vital feature for this project, [das Schwedische Konstruktikon, A.W.] since a cx [construction, A.W.] may be defined by constraints on different levels: word, word-form, part of speech, morphosyntactic category, grammatical function, intonation, information structure, etc. (Borin et al. 2012: 12)

Beispiele für solche Beschränkungen sind in der Vergangenheit vielfältig formuliert worden, für die way-Konstruktion und die reflexive Bewegungskonstruktion habe ich sie in Unterabschnitt 3.1.4 bereits diskutiert. Bei der Frage, wie solche Beschränkungen aussehen, fällt die Antwort meist sehr allgemein aus. So können „Beschränkungen (constraints) [...] sowohl semantischer als auch grammatischer Natur sein.“ (Ziem 2018e: 30). Borin et al. (2012: 12) zählen, wie soeben gesehen, noch zahlreiche weitere Ausprägungen auf. Für die way-Konstruktion und ihre deutschen Äquivalente sind es vor allem semantische Beschränkungen, die die Klasse der in ihre KE einsetzbaren KtE, insbesondere die Klasse der LE, die in 
das KE EREIGNIS eingesetzt werden können, bestimmen. ${ }^{37}$ Bisweilen werden solche Beschränkungen direkt als Bestandteil eines (mentalen) Konstruktikons aufgefasst: „Knowing a construction involves (i.a.) knowing what kinds of elements fit into the construction's open slots.“ (Verhagen 2009: 140). ${ }^{38}$ Reformuliert man dies für den Begriff der Präferenzen, wird deutlich, warum sie Teil eines semantischen Parameters sein und konstruktikographisch erfasst werden müssen.

Neben semantischen Präferenzen, die aus konstruktionssemantischer Sicht vor allem in Gestalt von Präferenzen für lexikalische Frames bei der Konstitution von Konstrukt-Frames zu verstehen sind, weisen Konstruktionen formale Beschränkungen auf, die etwa die grammatischen Kategorien der Instanzen ihrer Strukturelemente betreffen. Der Präferenzbegriff eignet sich für solche Phänomene nicht, da Beschränkungen wie diese gar nicht oder nur in sehr begrenztem Maße missachtet werden können und konstitutiv für eine Konstruktion und deren korpuslinguistische Identifikation sind. Diese Beschränkungen klammere ich aus der folgenden Diskussion aus, bis ich in Unterabschnitt 5.3.2 gesondert auf sie zu sprechen komme.

Die Tatsache, dass sich in der Literatur zur way-Konstruktion und ihren deutschen Äquivalenten beschriebene Beschränkungen durch Korpusanalysen teilweise widerlegen lassen, wie ich es in Unterabschnitt 3.1.4 für die reflexive Bewegungskonstruktion aufgezeigt habe, sollte Anlass zur Skepsis geben. Ich möchte deshalb dafür plädieren, aus einer gebrauchsbasierten Perspektive für semantische Phänomene weniger von Beschränkungen, sondern vorrangig von Präferenzen zu sprechen. Dies ist nicht nur sprachtheoretisch natürlicher (und plausibler), ${ }^{39}$ sondern insbesondere methodisch angebrachter. Eine sprachtheoretische Begründung scheint deshalb notwendig, weil der Präferenzbegriff bei

37 Engelberg et al. (2011: 96-101) argumentieren allerdings dafür, dass bei Argumentstrukturen auch andere KE (bei ihnen Slots genannt) als dasjenige, das für ein Verb vorgesehen ist, für Präferenzen infrage kommen. Bei den drei untersuchten Konstruktionen sind solche Präferenzen insbesondere für die Evokation einer emergenten Struktur relevant, weshalb ich darauf in Unterabschnitt 5.7.2 zurückkomme.

38 Vgl. für diese These auch Schneider (2014: 364) und Diedrichsen (2014: 185). Um solche Präferenzen zu erfassen, möchte Verhagen (2009: 139-142) den strukturalistischen Begriff des Paradigmas verwenden (vgl. auch Schneider 2014: 369). Dies ist allerdings eine rein theoretische Setzung, deren Bezugspunkt ausschließlich auf LE-Ebene zu operieren scheint (siehe meine Argumentation unten). Wie solche Paradigmen empirisch und damit konstruktikographisch zu beschreiben sind, ist damit noch nicht geklärt.

39 Sprachtheoretische Argumente für einen Präferenzbegriff finden sich über die von mir im Folgenden diskutierten Aspekte hinaus bei Feilke (1994: 162, 1998: 72). Eine Abgrenzung vom generativistischen Begriff der Selektionsrestriktionen zugunsten eines Präferenzbegriffs nimmt Hanks (1996: 79, 1997: 120, 2011: 499, 2013: 19) vor. 
der Diskussion um Konstruktionen und ihre semantischen Eigenschaften eine noch immer unterrepräsentierte Rolle zu spielen scheint. Eine Ausnahme ist dessen Verwendung von Engelberg et al. (2011: 93-104) und Engelberg (2019: 17-18), die die theoretischen Implikationen, die damit einhergehen, allerdings nicht reflektieren. Auch für Stefanowitsch (2008b: 257) „ist klar, dass jede Konstruktion ihre eigenen graduellen Präferenzen bezüglich der Verbklassen hat, die in ihr auftreten können“.

Spätestens seit Chomsky (1965: 95) die Unterscheidung in Selektions- und Subkategorisierungsregeln eingeführt hat, kann die Untersuchung von Beschränkungen als ein wesentlicher Teil vieler Syntaxtheorien gelten. ${ }^{40}$ Die Konstruktionsgrammatik ist, trotz ihrer Distanzierung von generativistischen Sichtweisen, in dieser Hinsicht keine Ausnahme. Welche Argumente sprechen gegen den Begriff der Beschränkung, Subkategorisierung oder Selektionsrestriktion und für den Begriff der Präferenz? Eine sprachtheoretische Antwort gibt Hanks (1996):

\footnotetext{
In the literature, such subcategorizational phenomena are often referred to as selectional restrictions. It is preferable, though, to think of them as selectional preferences. A restriction prevents or forbids you from doing something, whereas it is often the case that locutions excluded by a selectional preference are, nevertheless, perfectly grammatical, psychologically acceptable, and communicatively adequate. They are just not conventional. They deviate from an established norm. (Hanks 1996: 79) $)^{41}$
}

Hanks' Präferenzbegriff nimmt Bezug auf ein computerlinguistisches Modell von Wilks (1975, 1980), lässt sich aber ebenso, wie es seine Ausführungen andeuten, auf ein Grammatikmodell wie das der gebrauchsbasierten Konstruktionsgrammatik ausweiten. Hanks (2007: 132-133) unterscheidet drei Arten von Präferenzen: lexikalische Präferenzen, syntaktische Präferenzen und domänen-spezifische Präferenzen. In seiner Kritik am Begriff der Selektionsrestriktion scheint er überwiegend syntaktische Beschränkungen im Sinn zu haben, denen er einen Präferenzbegriff entgegensetzt. Syntaktische Beschränkungen (bzw. Präferenzen) aber sind nicht Kern des in der Konstruktionsgrammatik verwendeten Beschränkungsbegriffs, denn ihre (nach traditionellem Verständnis) Verletzung ist gerade ein kon-

40 So basieren etwa die Head-Driven Phrase Structure Grammar (HPSG, vgl. für einen Überblick Müller 2019: 263-309) oder das Simpler-Syntax-Modell von Culicover \& Jackendoff (2005) wesentlich auf der Annahme von Constraints.

41 Hanks liegt hiermit erstaunlich nah an der Auffassung, die Langacker für die Beurteilung ,neuer‘ sprachlicher Ausdrücke vertritt, die also gewissermaßen von Präferenzen abweichen: „Assessing their conventionality (or 'well-formedness') is a matter of categorization: categorizing judgments either sanction them as elaborations of schematic units or recognize them as departing from linguistic convention as currently established.“ (Langacker 1990: 16). 
stitutives Moment vieler syntaktischer Konstruktionen (vgl. Goldberg \& Casenhiser 2006: 344). ${ }^{42}$ Die reflexive Bewegungskonstruktion bietet ein illustratives Beispiel: Die Tatsache, dass LE wie arbeiten, kämpfen oder kaufen mitsamt ihrer lexikalischen Bedeutungen in Konstrukten erscheinen können, die Reflexiva und PP als ihre Argumente kodieren, erscheint als klarer Verstoß gegen intuitive Selektionsrestriktionen dieser LE. ${ }^{43}$ Die Konstruktionsgrammatik tritt als explizite Gegenspielerin solcher Annahmen an, da sie, ganz in Hanks' Sinne, mit einer Erklärung für die Grammatikalität und Akzeptabilität solcher Konstrukte aufwarten kann.

Im Gegenteil dazu sind es vielmehr semantische Beschränkungen, deren Annahme sich bis in die Konstruktionsgrammatik fortführt. Die schon erwähnten angenommenen Beschränkungen der way-Konstruktion sind ein Beispiel dafür. Dass aber auch sie nicht unproblematisch sind, zeigt die Tatsache, dass sie sich, wie in Unterabschnitt 3.1.4 gezeigt, korpusbasiert in vielen Fällen widerlegen lassen. Dies ist ein Argument dafür, von Präferenzen und nicht von Beschränkungen zu sprechen.

Wie lassen sich semantische Beschränkungen methodisch ermitteln? Die Antwort darauf lautet in der Regel: durch Intution, genauer: durch Grammatikalitätsund Akzeptabilitätsurteile. So beruhen die semantischen Beschränkungen der way-Konstruktion, die Jackendoff (1990: 213, 1997: 546) und Goldberg (1995: 199, 203-204) postulieren und die etwa Oya (1999: 357-358) auch für das Deutsche adaptiert, ausschließlich auf wenigen, konstruierten Beispielen. Dies ist insofern verwunderlich, als Goldberg (1995: 199-218, 1996) explizit Korpusdaten heranzieht, auf eine quantitative Auswertung allerdings verzichtet und gerade an den Stellen, an denen sie für die semantischen Beschränkungen der Konstruktion argumentiert, zwar nicht ausschließlich, aber dennoch stark Gebrauch von konstruierten Beispielen macht. Ein solches Vorgehen ist aus gebrauchsbasierter Sicht nicht zu halten. ${ }^{44}$ Gebrauchsbasiert lassen sich ausschließlich semantische Präferenzen untersuchen, keine semantischen Beschränkungen. Der Unterschied

42 Ähnlich argumentiert Coulson (2001: 275) aus frame-semantischer Perspektive, dass die Basis von Frame-Anpassungen (bei ihr: Frame-Shifting) in der Missachtung von Beschränkungen der Slots eines Frames liegt.

43 Auf die analoge Frage nach der Lizenzierung von Verbpartikeln in der reflexiven Partikelverbkonstruktion, die mit bestimmten Basisverben ansonsten distributionell nicht erwartbar wäre, komme ich in Unterabschnitt 6.4.2 zurück.

44 Sicher ist die Kritik von Welke (2019: 52) an Lasch (2016a: 3-4), dass ,gebrauchsbasiert ' nicht notwendigerweise mit ,korpusbasiert' gleichzusetzen ist, gerechtfertigt. Eine korpusbasierte Studie ist aber stets ,gebrauchsbasierter' als eine, die lediglich auf introspektiven Daten beruht. Allerdings ist, anders als von Welke (2021a: 374, Anm. 5) behauptet, kaum davon auszugehen, dass auch reine Introspektion als, gebrauchsbasiert‘ gelten kann. Um diese vorliegende Art gebrauchs- 
liegt nicht nur in der von Hanks (1996: 79) artikulierten sprachsystematischen Dichotomie zwischen ,Verboten“ (Beschränkungen) und ,Möglichkeiten` (Präferenzen), sondern in methodischer Hinsicht in der Beobachtbarkeit (vgl. Bücker 2012: 61-88): Präferenzen sind direkt an sprachlichem Material und quantitativen Auswertungen beobachtbar, Beschränkungen nicht. Beschränkungen lassen sich nur durch das Konstruieren introspektiver Daten scheinbar sichtbar machen. Die Frage nach der Akzeptabilität eines Beispiels ist demnach „only a weak indicator of the actual grammatical patterns in language use“ (Croft 2009a: 18).

Aus korpuslinguistischer Sicht ist ein Vorzug des Präferenzbegriffs somit angebracht. Wollte man Beschränkungen korpuslinguistisch untersuchen, müsste man aus der Untersuchung aller relevanten Belege des Korpus vom NichtVorliegen bestimmter Phänomene (z.B. LE, die als KtE ein KE instanziieren können) auf deren Ungrammatikalität oder Inakzeptabilität schließen. Dies aber ist freilich ein unangemessener Schluss, denn das Nicht-Vorliegen eines Phänomens in einem Korpus gibt keine Auskunft über das Nicht-Vorhandensein dieses Phänomens in einer Sprache. Grundsätzlich ist die Annahme, korpuslinguistische Untersuchungen gäben keine Auskunft über solche negative Evidenz/(, unangebracht. ${ }^{45}$ Wie Stefanowitsch $(2006,2008 a)$ argumentiert, lassen sich negative Evidenzen sehr wohl korpuslinguistisch untersuchen, sobald man akzeptiert, dass kein grundsätzlicher Unterschied zwischen der niedrigen Frequenz eines Phänomens und dem Nicht-Vorhandensein eines Phänomens in einem Korpus besteht: „[T]he non-occurrence of a particular linguistic structure is merely the limiting case; it is not qualitatively different from very rare occurrences." (Stefanowitsch 2006: 72). Somit lässt sich die Frequenz eines Phänomens mit seiner Grammatikalität oder Akzeptablität vergleichen:

The continuum between significantly rare and significantly absent structures is not fundamentally different from the continuum between various degrees of unacceptability that is regularly found for acceptability ratings. (Stefanowitsch 2006: 73)

Formuliert man beobachtete Phänomene hinsichtlich ihrer Präferenzen dergestalt, dass hohe Frequenzen (z.B. bestimmter LE, die als KtE ein KE instanziieren können) auf solche Präferenzen hinweisen, besteht neben ihrer direkten Beobachtbarkeit der Vorteil, die Untersuchung nicht auf hypothetischen

basierter Arbeit in den Facetten gebrauchsbasierter Ansätze, die Kemmer \& Barlow (2000) aufzeigen (vgl. auch Kapitel 1), zu verorten, könnte man von ,radikaler Gebrauchsbasiertheit‘ sprechen. 45 Diese Auffassung findet sich wohl zuerst bei Chomsky (2002: 15), der argumentiert, dass die Grammatikalität eines sprachlichen Phänomens nicht mit dessen Auftreten in einem Korpus und/oder dessen statistischer Signifikanz gleichzusetzen ist (vgl. dazu Stefanowitsch 2006: 61, 2008a: 515, 518). 
Grammatikalitäts- und Akzeptabilitätsurteilen beruhen lassen zu müssen, sondern Ergebnisse direkt aus dem beobachteten Daten abzuleiten. Dies gilt sowohl für positive Evidenz (hohe Frequenz, Indikator für Präferenzen) als auch für negative Evidenz (niedrige Frequenz, Indikator für Dispräferenzen). ${ }^{46}$ Dabei sind Präferenzen ebenso falsifizierbar: Selbstverständlich lassen sich aus den für ein Korpus festgestellten Präferenzen ebenso wenig Hypothesen für eine gesamte Sprache aufstellen, weitere Korpusanalysen aber können die einmal formulierten Präferenzen auf derselben methodischen Basis widerlegen oder bestätigen. Der korpuslinguistische Vorteil liegt auf der Hand: Schon ein einziger Beleg eines als ungrammatisch oder inakzeptabel angenommenen Phänomens kann dieses Urteil falsifizieren (vgl. Stefanowitsch 2006: 70). Genau dies lässt sich für die reflexive Bewegungskonstruktion an Belegen, die ich in Unterabschnitt 3.1.4 zitiert habe, nachvollziehen.

Innerhalb der konstruktionsgrammatischen Forschung ist bereits eine korpuslinguistische Methode zur Messung von Präferenzen entwickelt worden: die Kollostruktionsanalyse (Stefanowitsch \& Gries 2003, 2005; Gries \& Stefanowitsch 2004b; Stefanowitsch 2013). Die Kollostruktionsanalyse untersucht lexikalische Präferenzen, indem sie etwa diejenigen LE (Kollexeme) ermittelt, die bevorzugt und überzufällig ein KE einer Konstruktion instanziieren. Sie verzichtet allerdings meist auf Generalisierungen über diese LE, bezieht also deren lexikalische Frames und lexikalische Bedeutungen nicht ein (vgl. dazu aber die Diskussion in Unterabschnitt 8.5.1). Da aber gerade Präferenzen für bestimmte lexikalische Frames und lexikalische Bedeutungen wesentliche Eigenschaften einer Konstruktion sein können und als übergreifender Parameter mit anderen semantischen Parametern für Konstruktionen in Verbindung stehen, sehe ich von Kollostruktionsanalysen für die von mir untersuchten Konstruktionen ab und nehme die lexikalischen Frames als Ganze in den Blick. Wie ich in Unterabschnitt 8.5.1 argumentieren werde, ist ein solcher Ansatz einer Kollostruktionsanalyse gegenüber gerade durch seine inhärenten semantischen Generalisierungen im Vorteil.

\subsubsection{Formale (und einige semantische) Beschränkungen}

Die bisher diskutierte Kritik am Begriff der Beschränkung kreist vor allem um semantische Beschränkungen, die für einzelne Strukturelemente einer Konstruktion formuliert werden können. Ihn grundsätzlich durch den Begriff der Präferenzen zu ersetzen, ist methodisch sinnvoll, jedoch lassen sich für Konstruktionen

46 Zynischer formuliert: „[U]nlike acceptability judgments, negative corpus evidence meets the standards of scientific research.“ (Stefanowitsch 2006: 70). 
weiterhin Beschränkungen im engeren Sinne beobachten, die insbesondere formaler Natur sind, aber auch die semantischen Eigenschaften einer Konstruktion betreffen können. Formale Beschränkungen können die syntagmatische Reihenfolge von KE, deren grundsätzliche lexikalische Instanziierbarkeit sowie morphologische Restriktionen (z.B. auf bestimmte Flexionsformen eines KtE) betreffen (vgl. Boas, Lyngfelt \& Torrent 2019: 44). Semantische Beschränkungen können die Lizenzierung bestimmter KtE betreffen, die durch FE eines (lexikalischen oder Konstruktions-)Frames und/oder die semantischen Typen dieser FE beschränkt sein können (vgl. Ziem 2020a: 33). Da ich die Zusammenhänge zwischen KtE und FE als Strukturparallelen zwischen Konstruktionen und Frames in Kapitel 6 in den Blick nehme, gehe ich an dieser Stelle nicht auf sie ein.

Beschränkungen und Präferenzen der einzelnen Strukturelemente einer Konstruktion sind in jedem Fall aber ein wesentlicher Bestandteil konstruktikographischer Analysen und tragen maßgeblich zur Beschreibung dieser Strukturelemente bei (Unterabschnitt 7.3.1), ${ }^{47}$ sie sind aber ebenso für globale Eigenschaften einer Konstruktion von Belang. Für die drei untersuchten Konstruktionen möchte ich in diesem Unterabschnitt ihre wichtigsten formalen Beschränkungen formulieren und auch auf zentrale semantische Beschränkungen im engeren Sinne hinweisen.

Für die vier Strukturelemente der reflexiven Bewegungskonstruktion lassen sich einige recht enge formale Beschränkungen feststellen. Das KE BEwEGENDES ist noch am wenigsten beschränkt: Die KtE, die es instanziieren, können von unterschiedlichster Komplexität sein. Sie können in Gestalt eines Personalpronomens (44), als artikelloses Nomen im Plural oder als Eigennamen (45), als einfache NP mit Artikel im Singular (46) oder als komplexere, attributiv erweiterte NP (47) realisiert werden.

(44) $\{[$ Bewegendes Sie] arbeitete sich durch schulterhohes Dickicht\}; die Männer waren angeseilt, da sich unter dem Moosbewuchs tiefe Felsspalten verbargen; im stellenweise dichten Nebel hätte man sich ohne Kompaß leicht verirrt. (Schrott, Raoul: Tristan da Cunha oder die Hälfte der Erde; Hanser Verlag 2003, S. 31)

47 Das Schwedische Konstruktikon modelliert Beschränkungen und Präferenzen von Strukturelementen unter dem eigens dafür eingeführten Begriff des Kollostruktionselements (collostructional element), wenngleich die empirischen Ergebnisse nicht einer Kollostruktionsanalyse entspringen, sondern auf rohen Frequenzdaten basieren (vgl. Lyngfelt et al. 2012: 457; Sköldberg et al. 2013: 316; Lyngfelt et al. 2018: 90). Auch im Russischen Konstruktikon werden „,common fillers“ (Endresen \& Janda 2020: 4) von Strukturelementen dokumentiert. Noch weiter geht die konstruktikographische Forderung von Herbst (2016: 180-184, 2018a,b), der für den Aufbau eines Kollostruktikons plädiert. 
(45) a. \{[BewEgEndes Reporter] wühlten sich durch 1500 Seiten ärztlicher Diagnose und schenkten ihm dafür einen Freispruch ersten Grades mit Zitaten wie diesem: (Die Zeit, 20.01.2000, Nr. 4)

b. $\{$ [Bewegendes Phillip] drängelt sich durch die Menge $\}$. (Bach, Tamara: Marsmädchen, Hamburg: Verlag Friedrich Oetinger 2003, S. 132)

(46) \{[Bewegendes Der Vater] schlug sich durch die Haus- und Gartentür, die danach, und für Jahre, schräg in den Angeln hing\}. (Widmer, Urs: Das Buch des Vaters, Zürich: Diogenes 2004, S. 153)

(47) Unermüdlich \{wühlten sich [BEwEGENDEs ihre geschmeidigen Finger] durch das schwarze, schattenhafte Fleisch der erschlagenen Forellen\}, während das Gewitter immer wieder aufglomm und verlosch und schließlich in schmutziger, schummriger Dunkelheit unterging. (Düffel, John von: Vom Wasser, München: dtv 2006, S. 191)

Für die reflexive Partikelverbkonstruktion gilt grundsätzlich dasselbe. Auch ihr KE BEWEGENDES ist im Vergleich zu den anderen Strukturelementen am wenigsten beschränkt und erlaubt ebenfalls Personalpronomen (48) ebenso wie artikellose Nomen und Eigennamen (49), einfache NP mit Artikel (50) und komplexere NP (51).

(48) $\{[$ Bewegendes Ich] drückte mich durch die Leute zu der Treppe hindurch, die wir hochgekommen waren.\} (Goosen, Frank: Liegen lernen, Frankfurt am Main: Eichborn AG 2000, S. 212)

(49) a. Dort, wo sich die Brandung nicht wundwusch an dem rötlichen, von der Sonne gehärteten Stein, \{rankten sich [Bewegendes Muscheln] die Felswände hinauf\}. (Düffel, John von: Houwelandt, Köln: DuMont Literatur und Kunst Verlag 2004, S. 24)

b. Als schließlich die Besitzerin des Sport-Equipments selbst herausklettert, \{dreht sich [Bewegendes Anneliese] kreidebleich zu mir um\} und flüstert: (Noll, Ingrid: Ladylike, Zürich: Diogenes 2006, S. 229)

(50) $\{$ [Bewegendes Die Bündnisgrünen] rangen sich schließlich zur Bildungssteuer durch\}. (Die Zeit, 27.01.2000, Nr. 5)

(51) a. $\quad$ In ihr Befremden mischte sich [Bewegendes eine gewisse Portion Neugier] hinein\}, eine Art Wissensdrang, mag sein, das hat ihr die Sache erleichtert. (Venske, Regula: Marthes Vision, Frankfurt am Main: Eichborn Verlag 2006, S. 134)

b. $\{$ [Bewegendes Die zweite Schöpfung, die in der Enterprise nicht bloß aus Kontroll- und Waffensystemen, sondern auch aus Computermenschen mit Identitätsproblemen besteht und den Angriffen der 
intergalaktischen Maschinenmenschen namens „Borg“ im Dienste der „authentischen“ Menschen widerstehen muss]\}, entwickelt sich konstant, aber höchst bedrohlich in die Zukunft hinein. (Die Zeit, 10.02.2000, Nr. 7)

Für die reflexive Weg-Konstruktion ist die Beschreibung solcher Beschränkungen aufgrund der sehr kleinen Datenmenge (Unterabschnitt 3.4.1) einfacher, wenngleich dadurch nicht ausgeschlossen werden kann, dass andere Formen nicht grundsätzlich unmöglich sind. So spricht die Tatsache, dass zwar Personalpronomen (52) und Eigennamen (53), aber keine artikellosen Nomen im Plural belegt sind, nicht dafür, dass solche Instanziierungen grundsätzlich unmöglich sind. Für die reflexive Weg-Konstruktion sind indes ebenso einfache NP mit Artikel (54) sowie erweiterte NP (55) belegt.

(52) Was nun Crna Gora (Montenegro) betrifft, so beglückwünsche ich die Montenegriner, dass $\left\{\left[\left[_{\text {BEWEGENDES }}\right.\right.\right.$ sie] sich ungestört durch Serbien einen Weg nach Europa bahnen wollen\}, obwohl sie formale Bindungen an Serbien haben. (Archiv der Gegenwart, 2001 [2000])

(53) $\{[$ Bewegendes Paul $]$ mußte sich einen Pfad zum Holzhaus bahnen\}. (Koneffke, Jan: Paul Schatz im Uhrenkasten, Köln: DuMont Buchverlag 2000, S. 77)

(54) $\{[$ BEwEGendes Ein Mann] bahnt sich den Weg zur Bühne, zum Kabel $\}$, doch kurz davor füllt die Stimme wieder wutzitternd den Raum: (Die Zeit, 23.03.2000, Nr. 13)

(55) \{[Bewegendes Mein Schälmesser mit der dünnen Klinge] säbelt sich einen Weg durch die buschigen Petersilienköpfe\}, während ich überlege, ob es tatsächlich Köpfe oder doch Blätter oder gar Büschel heißt. (Riedel, Susanne: Eine Frau aus Amerika, Berlin: Berlin Verlag 2003, S. 106)

Da diese formalen Beschränkungen so gering sind, sind sie für eine konstruktikographische Analyse kaum relevant. Indes ist eine semantische Beschränkung, die für das KE BEWEGENDES jeder der drei Konstruktionen gilt, wesentlich entscheidender: Die Entität, auf die das KtE dieses KE referiert, muss dieselbe sein, auf die das KEE referiert. Das KtE des KE BEWEGENDES muss also Antezendens des als Reflexivum instanziierten KEE sein (vgl. Unterabschnitt 3.4.2). Bei dieser Eigenschaft handelt es sich um eine Beschränkung im engeren Sinne, da sie konstitutiv für die Konstruktion ist. Wird die Beschränkung missachtet, handelt es sich nicht 
mehr um dieselbe Konstruktion: Es handelt sich aus semantischer Sicht um eine konstitutive Eigenschaft der Konstruktion. ${ }^{48}$

Komplexer sind die Verhältnisse für das KE EREIGNIS, insbesondere in semantischer Hinsicht. In formaler Hinsicht ist dieses KE lediglich kategorial beschränkt: Es dürfen ausschließlich Verben bzw. VP darin instanziiert werden. ${ }^{49}$ Da sie als KtE den lexikalischen Frame evozieren, sind die semantischen Beschränkungen, die dieses KE auszeichnen, von entscheidender Relevanz für die Frage danach, ob ein gegebener Beleg überhaupt ein Konstrukt einer der drei Konstruktionen enthält (vgl. Unterabschnitt 8.4.2) und - damit zusammenhängend - ob in einen Konstrukt-Frame der Konstruktions-Frame Motion eingeht oder der lexikalische Frame allein ausreicht, um den Konstrukt-Frame zu konstituieren (vgl. Abschnitt 6.2).

Das KE EREIGNIS gibt eine einzige globale Voraussetzung vor, die Instanzen, die als Konstrukte insbesondere der reflexiven Bewegungskonstruktion und der reflexiven Partikelverbkonstruktion eingestuft werden sollen, erfüllen müssen. Die es instanziierenden KtE müssen entweder einen zum Konstruktions-Frame relatierten oder unrelatierten lexikalischen Frame (Abschnitt 5.4) evozieren, wobei Letzterer dann gemeinsam mit dem Konstruktions-Frame den Konstrukt-Frame konstituieren muss (vgl. Unterabschnitte 3.4.2 und 8.4.2). Relatierte lexikalische Frames sind solche, die entweder mit dem Konstruktions-Frame Motion identisch sind oder zu ihm in einer Frame-zu-Frame-Relation, genauer: in Frame-Nähe stehen. Unrelatierte lexikalische Frames sind alle anderen möglichen lexikalischen Frames unter der Voraussetzung, dass diese gemeinsam mit dem KonstruktionsFrame den Konstrukt-Frame konstituieren. Während man bei relatierten lexikalischen Frames noch von Beschränkungen sprechen kann, da ihre Klasse relativ deutlich umrissen werden kann (vgl. Unterabschnitt 5.4.3 für die zu Motion relatierten Frames), gilt dies für unrelatierte lexikalische Frames nicht. Als unrelatiertier lexikalischer Frame kommt jeder lexikalische Frame infrage, der gemeinsam mit dem Konstruktions-Frame den Konstrukt-Frame konstituieren kann bzw. der gemeinsam mit diesem in einem Konstrukt-Frame belegt ist. Beschränkungen gelten also nicht für die Klasse der unrelatierten Frames an sich, da die Konstitution eines ,neuen“ Konstrukts mit einem unrelatierten lexikalischen Frame gerade ein Ausdruck der Produktivität der Konstruktion ist (Abschnitt 5.6). Je produktiver die Konstruktion ist, desto weniger lassen sich Beschränkungen für unrelatierte le-

48 Vgl. dazu auch die Rolle des KEE bei der Evokation des Konstruktions-Frames durch die syntagmatische Kombination von KtE und KEE (Unterabschnitt 8.3.3).

49 In den Annotation berücksichtige ich jeweils ausschließlich das Vollverb. Modal- oder Hilfsverben zählen zwar grundsätzlich mit zu den KtE dieses KE, aus Gründen der Übersichtlichkeit klammere ich sie aus der Annotation aus. 
xikalische Frames formulieren. Für unrelatierte lexikalische Frames an sich sind demnach einzig Präferenzen formulierbar, anhand derer Fragen nach der Evokation des Konstruktions-Frames in einem Konstrukt beantwortet werden können, auf die ich in Abschnitt 8.3 zurückkomme.

Beschränkungen sind für das KE EREIGNIS also globaler zu formulieren: ihre KtE müssen a) entweder einen relatierten lexikalischen Frame evozieren oder b) einen unrelatierten lexikalischen Frame evozieren, dieser muss dann aber gemeinsam mit dem Konstruktions-Frame Motion den Konstrukt-Frame konstituieren. ${ }^{50}$ Wird diese globale Beschränkung nicht eingehalten, ist ein gegebener Beleg nicht als Konstrukt der reflexiven Bewegungskonstruktion oder reflexiven Partikelverbkonstruktion einzustufen. Mit anderen Worten: Es handelt sich dann um ein Falschpositiv. Die Belege in (56) sind Beispiele für Falschpositive der reflexiven Bewegungskonstruktion, die die globale Beschränkung des KE EREIGNIs missachten, die Belege in (57) illustrieren dies für die reflexive Partikelverbkonstruktion. ${ }^{51}$ Solche Falschpositive sind zudem von methodologischem Wert, um die Konstrukte einer Konstruktion und letztendlich ihren Konstruktions-Frame zu identifizieren (dazu Unterabschnitt 8.4.2).

(56) a. Martina strich sich durch das Haar, wie früher, aber mit einer älteren Hand. (Kuckart, Judith: Lenas Liebe, Köln: DuMont Literatur und Kunst Verlag 2002, S. 217)

b. Diese Furcht verwandelt sich mehr und mehr in ein Vorurteil, um nicht zu sagen, in eine Ausrede. (Die Zeit, 05.01.2000, Nr. 2)

c. Der Trend zur Spezialisierung ergibt sich vor allem aus dem zunehmend härteren Wettbewerb. (Die Zeit, 30.03.2000, Nr. 14)

(57) a. Ich sah mich nach fettarmem Jogurt um, der schwer zu finden, aber immerhin erhältlich war. (Die Zeit, 10.02.2000, Nr. 7)

b. Die marktradikaleren Vorstellungen der so genannten Chicago-Schule setzten sich durch. (Die Zeit, 17.02.2000, Nr. 8)

c. Schon mit Mitte Zwanzig schloß er die berühmte Kunsthochschule in Warschau ab, ging wegen des Klimas und Lichts nach Sizilien, richtete sich in einem ehemaligen Lagergebäude am Hafen von Palermo ein Atelier ein und begann ein Künstlerleben wie aus einem Kitsch-

50 Für die reflexive Partikelverbkonstruktion existiert allerdings die Ausnahme, dass zwei für sie (und die reflexive Bewegungskonstruktion) unrelatierte Frames den Konstrukt-Frame vollständig konstituieren können und diese Belege dennoch als ihre Konstrukte eingestuft werden können: Darauf habe ich bereits in Unterabschnitt 5.2.4 hingewiesen. In Unterabschnitt 6.4.3 komme ich auf diese Problematik zurück.

51 Zur Herkunft der Daten vgl. Unterabschnitt 3.4.2. 
film: Malen, Spaziergänge am Meer, Weintrinken, Mädchen. (Arjouni, Jakob: Chez Max, Zürich: Diogenes 2006, S. 13)

Interessant ist, dass für die reflexive Weg-Konstruktion im Gegensatz zu den anderen beiden Konstruktionen kaum solche Falschpositive festzustellen sind. Dies liegt an einer sehr konkreten Beschränkung ihres KE EREIGNIS. Es lässt für die überwiegenden Konstrukte einzig die LE bahnen zu. Wie bereits in Unterabschnitt 3.3.2 gesehen, ist in meinen Daten von dieser Beschränkung nur eine einzige Ausnahme belegt: Ein einziges Konstrukt der reflexiven Weg-Konstruktion weist eine andere LE auf, nämlich säbeln in (58).

\{Mein Schälmesser mit der dünnen Klinge [EREIGNIs säbelt] sich einen Weg durch die buschigen Petersilienköpfe\}, während ich überlege, ob es tatsächlich Köpfe oder doch Blätter oder gar Büschel heißt. (Riedel, Susanne: Eine Frau aus Amerika, Berlin: Berlin Verlag 2003, S. 106)

Eine Klasse von Strukturelementen aller drei Konstruktionen, für die sich weiterhin Beschränkungen im engeren Sinne formulieren lassen, ist die der KEE und KorE, bei Ersteren sowohl diejenigen, die als Reflexivum instanziiert werden, als auch das KEE RICHTUNG der reflexiven Partikelverbkonstruktion. Ihre Beschränkungen liegen in der Natur dieser Art von Strukturelementen, da die lexikalische Fixiertheit von KEE und KorE tendenziell über alle Konstrukte hinweg invariant ist und sie im einfachsten Fall durch nur ein einziges Element instanziierbar sind (dazu Unterabschnitte 2.3.1 und 3.1.2). Wie bereits in den Unterabschnitten 3.1.2, 3.2.2 und 3.3.2 erwähnt, bilden die drei untersuchten Konstruktionen in dieser Hinsicht eine gewisse Ausnahme, da ihre KEE sowie das KorE jeweils eine kleine Bandbreite unterschiedlicher Instanzen zulassen. Die Beschränkung ist im Falle der KEE und zum Teil auch für das KorE ähnlich wie beim KE EREIGNIS kategorialer Natur und zusätzlich morphologisch-paradigmatisch: Neben dem ,echten' Reflexivpronomen sich können im Falle der reflexiven Bewegungskonstruktion und der reflexiven Partikelverbkonstruktion noch die akkusativischen reflexiv gebrauchten Personalpronomen mich, dich (1. und 2. Person Singular), uns und euch (1. und 2. Person Plural) als KEE dienen. In der reflexiven Weg-Konstruktion stehen das ,echte' Reflexivum sich sowie die reflexiv gebrauchten Personalpronomen im Dativ. Daneben müssen die Personalpronomen noch die bereits für das KE BEWEGENDES erwähnte Voraussetzung erfüllen, koreferent mit dem KtE ebendieses KE zu sein, um als ,reflexiv gebrauchte‘ Personalpronomen gelten zu können.

Ähnlich kategorial beschränkt ist das KEE RICHTUNG der reflexiven Partikelverbkonstruktion. Möglich sind hier ausschließlich Verbpartikeln auf präpositionaler, adverbialer und adjektivischer Basis sowie einige Partikeln auf nominaler Basis. Die Datenauswahl habe ich in Abschnitt 3.4.1 begründet, in Tabelle 5.8 sind 
noch einmal alle untersuchten Partikeln in ihrer Klassifikation nach Duden (2016: 708-713) zusammengefasst. Die Validität dieser Beschränkungen lässt sich freilich nur durch größere Korpusstudien verifizieren, die zeigen müssen, dass andere Partikeln - etwa nominale wie acht, preis und stand - für die Konstruktion nicht belegbar sind..$^{52}$

Tab. 5.8: Beschränkungen des KEE RICHTUNG der reflexiven Partikelverbkonstruktion hinsichtlich Verbpartikeln (Klassifikation nach Duden 2016: 708-713)

\begin{tabular}{llll}
\hline Präpositional & Adverbial & Adjektivisch & Nominal \\
\hline$a b$ & fort & frei & heim \\
an & her & hoch & \\
auf & hin & & \\
aus & weg & & \\
bei & zurück & & \\
durch & & & \\
ein & & \\
hinter & & \\
mit & & \\
nach & & \\
über & & \\
um & & \\
unter & & \\
vor & & \\
zu & & \\
\hline
\end{tabular}

Innerhalb dieser Beschränkungen auf bestimmte Partikeln sind zudem Präferenzen beobachtbar, die sich nach der Frequenz der einzelnen Partikeln richten. Wie bereits aus Tabelle 3.5 in der Übersicht der Korpusdaten in Unterbschnitt 3.4.2 hervorgeht, weist die reflexive Partikelverbkonstruktion und mit ihr deren KEE RICHTUNG eine starke Präferenz für die Partikel um (als einfache Partikel oder Zweitglied von Doppelpartikeln) auf, die sowohl in Distanz- als auch in Kontaktstellung die frequenteste Partikel darstellt. An zweiter Stelle liegt die Partikel vor, an dritter Stelle $a b$ (jeweils ebenfalls inklusive Doppelpartikeln).

52 Anbieten würde sich hierfür eine Kollostruktionsanalyse, wie sie etwa Goschler \& Stefanowitsch (2010) in Form einer kovariierenden Kollexemanalyse für die Assoziationen zwischen einzelnen ,Bewegungsverben' und Verbpartikeln durchführen. Ihre Analyse ist aber gerade auf jene ,Bewegungsverben“ beschränkt und schenkt dem möglichen Auftreten eines Reflexivums keine Beachtung, sodass sich daraus keine Rückschlüsse für die reflexive Partikelverbkonstruktion ableiten lassen. 
Auch für das KorE der reflexiven Weg-Konstruktion lassen sich konkrete Beschränkungen festhalten. Sie sind gemischt kategorial-lexikalischer Natur, da es sich dabei um eine NP mit dem bevorzugten Kopf Weg oder einem semantisch verwandten Nomen handeln muss. Der weitere Aufbau der NP ist, wie bereits in Unterabschnitt 3.3.2 ausgeführt, relativ unbeschränkt. Er kann, wie die drei folgenden Belege noch einmal illustrieren sollen, aus einem Definitartikel (59), einem Indefinitartikel (60) oder einem Possessivartikel (61) bestehen (vgl. ähnlich Verhagen 2003a: 345, 2003c: 232-233; Mortelmans \& Smirnova 2020: 60).

(59) Wie ein Triumphator \{bahnt sich Schiller [KonE den Weg] durch die Menschenmenge\}, eskortiert von den Würdenträgern der Universität. (Safranski, Rüdiger: Friedrich Schiller, München Wien: Carl Hanser 2004, S. 311)

(60) \{Mein Schälmesser mit der dünnen Klinge säbelt sich [KoRE einen Weg] durch die buschigen Petersilienköpfe\}, während ich überlege, ob es tatsächlich Köpfe oder doch Blätter oder gar Büschel heißt. (Riedel, Susanne: Eine Frau aus Amerika, Berlin: Berlin Verlag 2003, S. 106)

(61) Jawohl, denn die Damen wirken „schwerfällig und lustlos“, \{bahnen sich an Bord nur mühsam [KoRE ihren Weg] durch die engen Gänge\} und ecken sehr wahrscheinlich auch bei solchen Passagieren an, die es in höchstem Maße unerquicklich finden, ihren Anisschnaps von einem Fesselballon serviert zu bekommen. (Die Zeit, 10.02.2000, Nr. 7)

Neben diesen formalen Beschränkungen weist das KorE der reflexiven WegKonstruktion eine semantische Beschränkung auf: Der Kopf der es instanziierenden NP muss durch das Nomen Weg oder ein semantisch verwandtes Nomen gebildet werden. Neben dem Nomen Weg sind noch, wie in (62) und (63) zu sehen, die Nomen Pfad und Trampelpfad belegt. Ihre semantische Verwandtschaft zum Nomen Weg zeigt sich darin, dass alle diese Nomen Frames evozieren, die in einer Frame-zu-Frame-Relation zum Konstruktions-Frame Motion stehen (vgl. Unterabschnitte 8.3.1 und 8.5.2).

(62) \{Paul mußte sich [KorE einen Pfad] zum Holzhaus bahnen\}. (Koneffke, Jan: Paul Schatz im Uhrenkasten, Köln: DuMont Buchverlag 2000, S. 77)

(63) $\{[$ KonE Der Trampelpfad], den Jorge sich in Jahren gebahnt hatte\}, war verschwunden, das Wasser hatte ihn genommen. (Düffel, John von: Houwelandt, Köln: DuMont Literatur und Kunst Verlag 2004, S. 293)

Aus formaler Perspektive ist ein Vergleich der drei Konstruktionen hinsichtlich der Beschränkungen des KE WEG bzw. 〈WEG〉 am interessantesten. Für die reflexive Bewegungskonstruktion und die reflexive Weg-Konstruktion ist dieses KE auf die 
Instanziierung durch PP beschränkt. Darüber hinaus muss die in diese PP eingebettete NP im Akkusativ stehen, wenn es sich bei der Präposition um eine Wechselpräposition handelt (vgl. Unterabschnitt 3.4.2). Präpositionen, die nur Akkusativ regieren, sind unkritisch. Das KE 〈WEG〉 der reflexiven Partikelverbkonstruktion weist die kategoriale Beschränkung auf eine PP nicht auf. Wie bereits in Unterabschnitt 3.2.2 gezeigt, sind neben PP - die zweifelsfrei den Großteil der Konstrukte ausmachen - auch NP und Nebensätze möglich. Die hier noch einmal zitierten Konstrukte sollen illustrieren, dass sowohl NP im Akkusativ (64) als auch im Dativ (65) möglich sind (vgl. dazu auch Olsen 1996a: 274, 1999: 232-233). Steht im Konstrukt das KtE des KE EREIGNIS im $z$-Infinitiv, kann das KE 〈WEG〉 durch einen Nebensatz ohne Korrelat (66) oder mit Korrelat (67) instanziiert werden.

(64) Katharina fühlt die Tränen im Hals, die sie nicht hochkommen lassen will, sie kratzen und beißen und \{ätzen sich $\left[\left\langle\mathrm{WEG}_{\mathrm{WE}}\right.\right.$ die Speiseröhre] hinab\} - den Ösophagus -, wo ist denn ihre Mutter, warum darf sie sie nicht einmal trösten? (Dölling, Beate: Hör auf zu trommeln, Herz, Weinheim: Beltz \& Gelberg 2003, S. 202)

(65) In gewisser Weise, antwortete er und sah erst gründlich aus dem Fenster, bevor $\left\{\right.$ er sich [ $\left.{ }_{\langle\mathrm{WEG}\rangle} \mathrm{mir}\right]$ wieder zuwandte\}. (Schmitter, Elke: Frau Sartoris, Berlin: BvT 2000[2002], S. 118)

(66) Als das Spiel fertig war, wollte Nintendo den Game-Boy gerade wieder vom Markt nehmen, weil er veraltet war, und dass \{man sich im Sommer 1996 überhaupt herabließ, [〈WEG $\rangle$ dieses merkwürdige Spiel zu vertreiben]\}, geschah in der vagen Hoffnung, an den Erfolg des virtuellen Computervogels Tamagotchi anknüpfen zu können. (Die Zeit, 23.03.2000, Nr. 13)

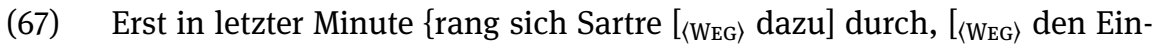
marsch in Budapest 1956 zu verurteilen]\}, während sieben Jahre später die Sowjetunion wieder „das einzige Land ist, wo das Wort Fortschritt noch einen Sinn hat“. (Die Zeit, 13.04.2000, Nr. 16)

Neben diesen kategorialen Beschränkungen lassen sich für die reflexive Bewegungskonstruktion und ihr KE WEG einige formale Eigenschaften beobachten, die eher als Präferenzen zu verstehen sind. Für das KE WEG sind Präferenzen für bestimmte Präpositionen dokumentiert, die als Kopf der instanziierenden PP infrage kommen. Wie bereits Smirnova (2018: 38) bemerkt, ist eine „eindeutige Präferenz für die Präposition durch“ erkennbar. Meine Daten bestätigen diesen Befund grundsätzlich, zeigen aber ein differenzierteres Bild. Wie bereits aus Tabelle 3.4 in Unterbschnitt 3.4.2 hervorgeht, liegt durch von allen für die reflexive Bewegungs- 
Tab. 5.9: Beschränkungen der Strukturelemente der reflexiven Bewegungskonstruktion

\begin{tabular}{|c|c|}
\hline Strukturelement & Beschreibung \\
\hline BEWEGENDES & $\begin{array}{l}\text { Die Entität, auf die ein KtE dieses KE referiert, muss mit derjenigen, auf die } \\
\text { das KEE referiert, identisch sein. }\end{array}$ \\
\hline EREIGNIS & $\begin{array}{l}\text { Die LE, die das KtE dieses KE bildet, muss einen relatierten oder unrelatier- } \\
\text { ten lexikalischen Frame evozieren. Im Falle eines unrelatierten lexikalischen } \\
\text { Frames muss dieser gemeinsam mit dem Konstruktions-Frame Motion den } \\
\text { Konstrukt-Frame konstituieren. }\end{array}$ \\
\hline KEE & $\begin{array}{l}\text { Das KEE muss durch ein Reflexivum (im weitesten Sinne), also ein ,echtes“ } \\
\text { Reflexivpronomen oder reflexiv gebrauchtes Personalpronomen in } 1 . \text { oder } 2 \text {. } \\
\text { Person instanziiert werden. Handelt es sich um Letzteres, muss es auf diesel- } \\
\text { be Entität wie das KtE von BEWEGENDES referieren. Auch das Reziprokprono- } \\
\text { men einander ist belegt. }\end{array}$ \\
\hline WEG & $\begin{array}{l}\text { Die KtE dieses KE müssen als PP realisiert werden, deren Präpositionen, } \\
\text { wenn es sich um Wechselpräpositionen handelt, Akkusativ regieren. }\end{array}$ \\
\hline
\end{tabular}

konstruktion untersuchten Präpositionen hinsichtlich der Anzahl relevanter Konstrukte auf dem fünften Platz, frequenter sind in, über, zu und auf.

Die für alle drei Konstruktionen ermittelten formalen und semantischen Beschränkungen sind in den folgenden drei Tabellen, für die reflexive Bewegungskonstruktion in Tabelle 5.9, für die reflexive Partikelverbkonstruktion in Tabelle 5.10 und für die reflexive $\mathrm{Weg}$-Konstruktion in Tabelle 5.11, zusammengefasst. Diese Zusammenfassungen sind Grundlage für die Definitionen der Strukturelemente in den drei Konstruktionseinträgen im Zusatzmaterial (vgl. auch Unterabschnitt 7.1.3).

\subsubsection{Präferenzen für lexikalische Frames}

Neben den vorrangig formalen Beschränkungen, die ich in Unterabschnitt 5.3.2 thematisiert habe, spielt eine bestimmte Art von Präferenzen für eine Konstruktionssemantik eine entscheidende Rolle: Präferenzen für lexikalische Frames. Daran schließen sich unmittelbar Fragen danach an, welche Relevanz solchen Präferenzen für die konstruktikographische Analyse zukommt und wie sie mit anderen Parametern in Verbindung stehen. Diesen möchte ich im Folgenden nachgehen, indem ich Präferenzen für lexikalische Frames als einen übergeordneten Parameter verstehe, der selbst zwar keinen Eingang in einen Konstruktionseintrag finden muss (Unterabschnitt 7.1.3), aber eine wichtige Datengrundlage für die Messung anderer Parameter liefert. 
Tab. 5.10: Beschränkungen der Strukturelemente der reflexiven Partikelverbkonstruktion

\begin{tabular}{|c|c|}
\hline Strukturelement & Beschreibung \\
\hline EWEGENDES & $\begin{array}{l}\text { Die Entität, auf die ein KtE dieses KE referiert, muss mit derjenigen, auf die } \\
\text { das KEE referiert, identisch sein. }\end{array}$ \\
\hline EREIGNIS & $\begin{array}{l}\text { Die LE, die das KtE dieses KE bildet, muss muss einen relatierten oder unre- } \\
\text { latierten lexikalischen Frame evozieren. Im Falle eines unrelatierten lexikali- } \\
\text { schen Frames muss dieser gemeinsam mit dem Konstruktions-Frame Motion } \\
\text { den Konstrukt-Frame konstituieren. Manche unrelatierten Frames sind aber } \\
\text { auch in der Lage, einen Konstrukt-Frame vollständig zu konstituieren, des- } \\
\text { sen Konstrukt gleichzeitig aber noch der reflexiven Partikelverbkonstruktion } \\
\text { zuzuordnen ist (vgl. Unterabschnitt 6.4.3). }\end{array}$ \\
\hline KEE & $\begin{array}{l}\text { Das KEE muss durch ein Reflexivum (im weitesten Sinne), also ein ,echtes“ } \\
\text { Reflexivpronomen oder reflexiv gebrauchtes Personalpronomen in } 1 . \text { oder } 2 . \\
\text { Person instanziiert werden. Handelt es sich um Letzteres, muss es auf diesel- } \\
\text { be Entität wie das KtE von BEWEGENDES referieren. }\end{array}$ \\
\hline RICHTUNG & $\begin{array}{l}\text { Dieses zweite KEE muss durch eine zum Basisverb als KtE des KE EREIGNIS } \\
\text { in Distanz- oder Kontaktstellung realisierte Verbpartikel instanziiert werden, } \\
\text { wobei alle Arten von Partikeln, präpositionale, adverbiale, adjektivische und } \\
\text { nominale (vgl. Duden 2016: 708), infrage kommen. }\end{array}$ \\
\hline$\langle\mathrm{WEG}\rangle$ & $\begin{array}{l}\text { Als Nicht-Kern-KE muss }\langle\text { WEG }\rangle \text { nicht in jedem Konstrukt instanziiert sein. Wird } \\
\text { es instanziiert, können seine KtE als PP (mit Akkusativ oder Dativ regieren- } \\
\text { den Präpositionen), NP im Akkusativ oder Dativ sowie als Nebensatz mit zu- } \\
\text { Infintiv realisiert werden. }\end{array}$ \\
\hline
\end{tabular}

Tab. 5.11: Beschränkungen der Strukturelemente der reflexiven Weg-Konstruktion

\begin{tabular}{ll}
\hline Strukturelement & Beschreibung \\
\hline BEWEGENDES & $\begin{array}{l}\text { Die Entität, auf die ein KtE dieses KE referiert, muss mit derjenigen, auf die } \\
\text { das KEE referiert, identisch sein. }\end{array}$ \\
EREIGNIS & $\begin{array}{l}\text { Die LE, die das KtE dieses KE bildet, muss einen relatierten oder unrelatier- } \\
\text { ten lexikalischen Frame evozieren. Im Falle eines unrelatierten lexikalischen }\end{array}$ \\
& Frames muss dieser gemeinsam mit dem Konstruktions-Frame Motion den \\
& Konstrukt-Frame konstituieren. \\
KEE & Das KEE muss durch ein Reflexivum (im weitesten Sinne), also ein ,echtes“ \\
& Reflexivpronomen oder reflexiv gebrauchtes Personalpronomen in 1. oder 2. \\
& Person instanziiert werden. Handelt es sich um Letzteres, muss es auf die- \\
& selbe Entität wie das KtE von BeWEGENDES referieren. Das Reflexivum steht \\
& dabei immer im Dativ. \\
& Das KorE muss als NP mit dem Nomen Weg oder einem semantisch verwand- \\
& ten Nomen als Kopf instanziiert werden, dem entweder ein Definitartikel, In- \\
KorE & definitartikel oder Possessivartikel vorangeht. \\
& Die KtE dieses Nicht-Kern-KE werden als PP realisiert, deren Präpositionen, \\
& wenn es sich um Wechselpräpositionen handelt, Akkusativ regieren. Als \\
& Nicht-Kern-KE muss dieses KE nicht in jedem Konstrukt instanziiert werden.
\end{tabular}


In Unterabschnitt 5.3.2 habe ich die Strukturelemente der drei Konstruktionen hinsichtlich ihrer formalen Beschränkungen untersucht. Ein Strukturelement, das in semantischer Hinsicht und für Präferenzen besonders zentral ist, ist das KE EREIGNIS aller drei Konstruktionen. Semantische Präferenzen, die mit diesem einhergehen, betreffen die darin einsetzbaren KtE, also diejenigen LE, die einen lexikalischen Frame evozieren können. Dass die lexikalischen Bedeutungen, die aus diesen Frames hervorgehen können, für semantische Eigenschaften wie die Polysemie einer Konstruktion von besonderer Relevanz sind, ist durch die Analyse der vier Lesarten der drei untersuchten Konstruktionen in Unterabschnitt 5.2.3 deutlich geworden. ${ }^{53}$ Dass ich dabei nicht allein auf der Basis von LE, also den KtE des KE EREIGNIS argumentiert habe, sondern nach lexikalischen Frames und lexikalischen Bedeutungen differenziert habe, ist kein Zufall. Ein wesentlicher Vorteil gegenüber einer Analyse, die sich allein auf die Formseite von LE, also KtE, stützt, liegt, wie in Unterabschnitt 8.5.1 näher zu begründen sein wird, darin, dass lexikalische Bedeutungen und insbesondere lexikalische Frames eine inhärente Generalisierung über LE hinweg darstellen. ${ }^{54}$ Generalisierungen auf der Ebene lexikalischer Bedeutungen sind dabei noch unterhalb von solchen auf der Ebene lexikalischer Frames anzusiedeln. Unterhalb dieser sind wiederum Generalisierungen anzusiedeln, die allein auf LE, für Argumentstruktur-Konstruktionen also zumeist Verben, beruhen. Diese Zusammenhänge sind in Abbildung 5.3 dargestellt.

Gerade für konstruktikographische Zwecke sind Generalisierungen, die nicht allein auf LE, sondern auf lexikalischen Frames beruhen, sinnvoll, da sich diese direkt dem Konstruktions-Frame gegenüberstellen lassen. Präferenzen für lexikalische Frames zu ermitteln trägt also durch die damit einhergehende Generalisierung wesentlich zur konstruktikographischen Beschreibung einer Konstruktion bei. Aus theoretischer Sicht deutet Hanks (2007) an, dass Präferenzen eine weitaus größere Tragweite zukommt als lediglich bestimmte Eigenschaften oder gar Idiosynkrasien einer LE oder einer Konstruktion zu erklären:

Preferences are everywhere in language systems. Preferences govern both meaning and linguistic behavior. That is to say, the entire process of constructing an interpretation for a linguistic utterance is governed by a contextual network of interacting preferences. (Hanks 2007: 132)

53 Aus valenztheoretischer Perspektive argumentiert Herbst (2018b: 10) im Einklang mit dieser Annahme, dass der Verb-Slot einer Argumentstruktur-Konstruktion (hier also das KE EREIGNIS) zentraler für die Charakterisierung einer Konstruktion ist als ihre anderen Slots (hier also etwa die KE BEWEgENDES oder WEg bzw. 〈WEG〉).

54 Zur konstruktikographischen Relevanz solcher Generalisierungen, die unterschiedliche (nicht nur mit Frames operierende) Formen annehmen können, vgl. Herbst (2018b: 12). 


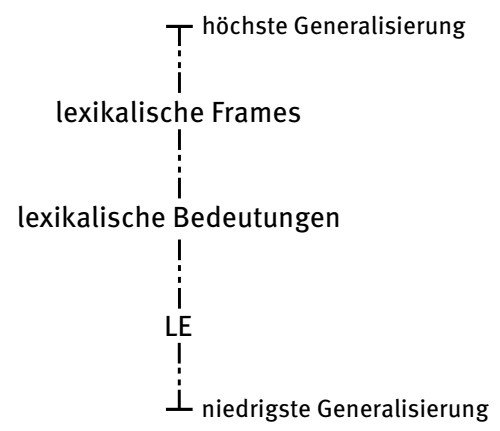

Abb. 5.3: Stärken von Generalisierungen über LE, lexikalische Bedeutungen und lexikalische Frames

Warum sind Präferenzen für lexikalische Frames ein übergeordneter Parameter? Ihre zentrale Stellung geht aus Abbildung 5.1 im Eingang dieses Kapitels hervor: Sie differenzieren nicht nur zu einem großen Anteil die Polysemie einer Konstruktion (Unterabschnitt 5.2.2), sondern sie stehen ebenso mit den semantischen Parametern der Frame-Nähe (Abschnitt 5.4), des Koerzionspotenzials (Abschnitt 5.5) und der Produktivität (Abschnitt 5.6) in Verbindung. Des Weiteren geben Präferenzen für lexikalische Frames Aufschluss über Fragen zu den Relationen zwischen Konstruktionen und Frames, etwa über den Mechanismus zur Evokation eines Konstruktions-Frames (dazu Unterabschnitt 8.1.2). Präferenzen für lexikalische Frames stellen sich in mindestens folgenden Aspekten als relevant heraus.

- Sie lösen konstruktionelle Ambiguität auf, ein Mechanismus, der üblicherweise für Verbklassen postuliert wird (vgl. z.B. Goldberg 2002: 335). ${ }^{55}$ Die Generalisierung von Verben hinsichtlich ihrer lexikalischen Frames stellt also eine Alternative zu einem Ansatz dar, der mit Verbklassen arbeitet (vgl. Unterabschnitt 8.4.2).

- Sie steuern den Mechanismus zur Evokation des Konstruktions-Frames. Der Anteil, den der Konstruktions-Frame an der Konstitution des KonstruktFrames hat, wird wesentlich darüber bestimmt, ob der lexikalische Frame allein in der Lage ist, alle KtE zu motivieren oder ob der Konstruktions-Frame hinzukommt (vgl. Unterabschnitt 8.2.2).

- Damit verbunden lassen sich über lexikalische Frames diejenigen Belege identifizieren, die Konstrukte einer gegebenen Konstruktion enthalten. Es

55 Gleiches gilt für konstruktionelle Polysemie, die sich ebenfalls über Verbklassen definieren lässt (vgl. z.B. Goldberg 1992: 50-55; Croft 2003: 56; Perek 2014: 72-82, 2015: 111-142). Wie sich die Polysemie einer Konstruktion über lexikalische Bedeutungen differenzieren lässt, ist in Unterabschnitt 5.2.3 deutlich geworden. 
kommen nämlich nur diejenigen Instanzen infrage, die durch bestimmte lexikalische Frames disambiguiert sind oder einen eindeutigen Anteil des Konstruktions-Frames an den Konstrukt-Frames erkennen lassen (vgl. Unterabschnitte 5.4.2 und 8.4.2.). ${ }^{56}$

Präferenzen für lexikalische Frames sind außerdem ein übergeordneter Parameter, weil sie in Verbindung zu mehreren anderen semantischen Parametern von Konstruktionen stehen und innerhalb dieser als methodischer Fixpunkt dienen. Diese Beziehungen möchte ich abschließend zusammenfassen und kommentieren. Neben den noch zu diskutierenden Parametern weise ich hierbei noch einmal auf die Relevanz von Präferenzen für lexikalische Frames für die beiden bereits betrachteten Parameter der formalen Abstraktheit und der konstruktionellen Polysemie hin.

- Die formale Abstraktheit einer Konstruktion (Abschnitt 5.1) lässt Rückschlüsse auf die Präferenzen für lexikalische Frames zu: Je abstrakter eine Konstruktion ist, desto eher ist zu erwarten, dass eine große Bandbreite lexikalischer Frames in ihre Konstrukt-Frames eingehen kann. Umgekehrt ist zu erwarten, dass die Präferenzen für lexikalische Frames bei einer wenig abstrakten Konstruktion stark eingeschränkt sind.

Die Analysen der drei Konstruktionen unterstützten diesen Befund. So weist die reflexive Bewegungskonstruktion eine große Bandbreite lexikalischer Frames auf, die von relatierten Frames wie Motion (68), Self_motion (69) oder Cause_motion (70) bis hin zu unrelatierten Frames wie Ingestion (71), Work (72) oder Scouring (73) reicht.

(68) Ich [Motion bewege] mich durch die Wohnung, es ist warm. (Braun, Marcus: Hochzeitsvorbereitungen, Berlin: Berlin Verlag 2003, S. 86)

(69) Die Morgensonne [self_motion stahl] sich schon durch die Ritzen der Fensterläden, aber in dem großen Haus war es so still, als schliefen selbst die Bücher in den Regalen. (Funke, Cornelia: Tintenherz, Hamburg: Cecilie Dressler Verlag 2003, S. 94)

56 In diesem Sinne bindet Ziem (2018a: 9) die Frage nach den typischen Füllungen eines KE an deren Prototypikalität, welche, wie ich in Unterabschnitt 5.4.2 zeige, darüber entscheidet, ob ein gegebener Beleg ein Konstrukt der angenommenen Konstruktion enthält oder nicht. Für die reflexive Partikelverbkonstruktion allerdings gilt, wie bereits angesprochen, eine Ausnahme, auf die ich in Unterabschnitt 6.4.3 zu sprechen komme. 
(70) Katja und Aleksej [Cause_motion schubsten] sich gegenseitig durch die Reihe, um anzukommen, aber nicht als erster. (Franck, Julia: Lagerfeuer, Köln: DuMont Literatur und Kunst Verlag 2003, S. 297)

(71) Abends las ich, die Bücherei war ziemlich umfangreich, und ich [Ingestion fraß] mich durch alles; langsam, genügsam, manchmal mit wirklicher Anteilnahme. (Schmitter, Elke: Frau Sartoris, Berlin: BvT 2000[2002], S. 35)

(72) Sie hörte das knarrende Geräusch, als die Scherenblätter aufgingen, spürte das Metall an ihrem Hals und dann [work arbeitete] sich die Schere säbelnd und schabend durch ihren Zopf. (Pressler, Mirjam: Malka Mai, Weinheim Basel: Beltz \& Gelberg 2001, S. 269)

(73) Unermüdlich [scouring wühlten] sich ihre geschmeidigen Finger durch das schwarze, schattenhafte Fleisch der erschlagenen Forellen, während das Gewitter immer wieder aufglomm und verlosch und schließlich in schmutziger, schummriger Dunkelheit unterging. (Düffel, John von: Vom Wasser, München: dtv 2006, S. 191)

Demgegenüber weist die reflexive Weg-Konstruktion, die, wie in Unterabschnitt 5.1.2 gesehen, weniger abstrakt ist, sehr eingeschränkte Präferenzen auf (Unterabschnitt 5.3.2): Neben dem einzig belegten realtierten lexikalischen Frame Motion (74) ist lediglich der unrelatierte Frame Cutting (75) belegt.

(74) Im Zickzack [Motion bahne] ich mir den Weg ins DB-Reisezentrum. (Die Zeit, 20.04.2000, Nr. 17)

(75) Mein Schälmesser mit der dünnen Klinge [cutting säbelt] sich einen Weg durch die buschigen Petersilienköpfe, während ich überlege, ob es tatsächlich Köpfe oder doch Blätter oder gar Büschel heißt. (Riedel, Susanne: Eine Frau aus Amerika, Berlin: Berlin Verlag 2003, S. 106)

- Konstruktionelle Polysemie ist unter anderem über lexikalische Frames und lexikalische Bedeutungen differenzierbar (Unterabschnitt 5.2.2). Eine einzelne Lesart kann Präferenzen für bestimmte lexikalische Frames, stärker aber noch für lexikalische Bedeutungen zeigen, also durch diese ausgelöst werden.

Die Analyse in Unterabschnitt 5.2.3 hat diese Zusammenhänge für die reflexive Beweungskonstruktion und die reflexive Partikelverbkonstruktion aufgezeigt. Zieht man zum Vergleich die reflexive Weg-Konstruktion heran, die wesentlich schwächer ausgeprägte Präferenzen aufweist, leuchtet es ein, dass ihre Polysemie kaum in dem Maße wie für die anderen beiden Konstruktionen ausgeprägt ist (vgl. 
dazu die Ergebnisse in Unterabschnitt 7.2.2). Über die lediglich zwei belegten lexikalischen Frames Motion und Cutting, die in (74) und (75) illustriert sind, lässt sich konstruktionelle Polysemie viel weniger feststellen als für eine Konstruktion mit ausgeprägteren Präferenzen für lexikalische Frames, wie dies für die reflexive Bewegungskonstruktion und die reflexive Partikelverbkonstruktion der Fall ist.

- Über Präferenzen für lexikalische Frames können unter Einbezug der FrameNähe zwischen dem Konstruktions-Frame und lexikalischen Frames diejenigen lexikalischen Frames ermittelt werden, die relatiert sind (vgl. auch Ziem 2018a: 9). Gleichzeitig können unrelatierte lexikalische Frames davon abgegrenzt werden - ein wichtiger Faktor für die Messung der Produktivität einer Konstruktion (vgl. Abschnitte 5.4 und 5.6).

Die oben zitierten Belege (68)-(70) einerseits und (71)-(73) andererseits zerfallen in zwei Gruppen: Die lexikalischen Frames, die zur ersten Gruppe gehören, können als relatiert klassifiziert werden, weil sie in (direkten oder indirekten) Frame-zu-Frame-Relationen zum Konstruktions-Frame Motion stehen. Für die lexikalischen Frames der zweiten Gruppe ist dies nicht der Fall: Sie stehen in keinen Frame-zu-Frame-Relationen zu Motion und sind deshalb als unrelatiert zu klassifizieren. Diese Klassifikation ist nur durch die Betrachtung von Präferenzen für lexikalische Frames möglich.

- Die Ausprägung der Präferenzen für lexikalische Frames bestimmt die Höhe des Koerzionspotenzials der Konstruktion (Abschnitt 5.5). Da Koerzionseffekte nur für eine bestimmte Klasse lexikalischer Frames angenommen werden können, sind Präferenzen ein Indikator dafür, ob eine Konstruktion häufig Koerzionseffekte hervorruft oder nicht.

In Konstrukten wie denen in (68)-(70) liegt keine Koerzion vor: Der lexikalische Frame wird im Konstrukt-Frame nicht durch den Konstruktions-Frame angepasst. Dies liegt daran, dass relatierte lexikalische Frames nicht von Koerzionseffekten betroffen sind, da sie stets vollständig einen Konstrukt-Frame bilden können und dieser dann keinen Anteil des Konstruktions-Frames besitzt (vgl. Unterabschnitt 6.2.1). Bei unrelatierten lexikalischen Frames wie denen in (71)-(73) jedoch tritt im Konstrukt-Frame zum lexikalischen Frame in den allermeisten Fällen der Konstruktions-Frame hinzu, was zu einem Koerzionseffekt führt. Präferenzen für (relatierte oder unrelatierte) lexikalische Frames bilden also die Grundlage für Aussagen über die Verteilung solcher Koerzionseffekte über die Konstrukte einer Konstruktion hinweg (vgl. Unterabschnitte 7.4.1 und 7.4.3). 
- Die Präferenzen für lexikalische Frames einer Konstruktion sind Ausdruck ihrer Produktivität (Abschnitt 5.6): Je diverser die Präferenzen beschaffen sind und je mehr unrelatierte lexikalische Frames belegt sind, desto produktiver ist die Konstruktion einzustufen (vgl. auch Ziem 2018a: 10).

Ein Vergleich zwischen reflexiver Bewegungskonstruktion und reflexiver WegKonstruktion mag dies erneut verdeutlichen: Schon an den wenigen Konstrukten mit unrelatierten lexikalischen Frames in (71)-(73) wird deutlich, dass allein dadurch, dass bereits mehrere unterschiedliche unrelatierte lexikalische Frames belegt sind, ein Unterschied zur reflexiven Weg-Konstruktion bestehen muss, für die nur ein einziger unrelatierter lexikalischer Frame, nämlich Cutting in (75), belegt ist (vgl. die Ergebnisse in 7.5.2). Dadurch erscheint die reflexive Bewegungskonstruktion deutlich produktiver als die reflexive Weg-Konstruktion.

- Die Evokation einer emergenten Struktur (Abschnitt 5.7) wird zum Teil über Präferenzen für lexikalische Frames und lexikalische Bedeutungen gesteuert: Konstrukte, deren Konstrukt-Frames sich aus bestimmten lexikalischen Frames zusammensetzen, evozieren eine emergente Struktur eher als solche mit anderen lexikalischen Frames.

Eine ausführliche Analyse, die die Rolle von lexikalischen Frames und lexikalischen Bedeutungen hinsichtlich der emergenten Struktur deutlich macht, folgt in Unterabschnitt 5.7.2. An dieser Stelle sei nur so viel gesagt, dass sich die Frage nach der Evokation einer emergenten Struktur unter anderem dadurch entscheidet, welcher lexikalische Frame an der Konstitution eines Konstrukt-Frames beteiligt ist und ob ein bestimmtes FE dieses lexikalischen Frames (oder wahlweise des Konstruktions-Frames) einen Standardwert trägt, also eine bestimmte lexikalische Bedeutung hervorbringt.

\subsection{Frame-Nähe}

Konstituiert sich ein Konstrukt-Frame aus lexikalischem Frame und KonstruktionsFrame, so unterscheiden sich diese beiden Frames mehr oder weniger deutlich voneinander. Ein Konstrukt ist damit in der Lage, zwei Frames miteinander zu verknüpfen, die auf den ersten Blick in keiner Beziehung zueinander stehen. Die Ausprägung einer solchen conceptual distance (im Sinne von Haiman 1983: 783, 1985: 102-147) entscheidet darüber, inwieweit die semantische Leistung des Konstruktions-Frames - im Vergleich zu der des lexikalischen Frames - in einem Konstrukt-Frame zu Tage tritt. Sie entscheidet darüber, inwiefern der lexikalische 
Frame durch den Konstruktions-Frame im Zuge einer Frame-Anpassung (Unterabschnitt 4.4.1) angepasst wird.

Gerade die Tatsache, dass in den folgenden Belegen lexikalische Frames wie Prevarication (76), Hostile_encounter (77) oder Perception_experience (78), die durch die KtE des KE EREIGNIS evoziert werden, in keinerlei Verbindung zum Konstruktions-Frame Motion stehen, lässt die Konstrukte als besonders ,kreativ‘, ${ }^{57}$ eben als besonders deutliche Konstrukte der reflexiven Bewegungskonstruktion und die Konstruktion selbst als produktiv erscheinen.

(76) Niemand hier kann was Konkretes, alle wurschteln bloß rum, saugen Comedy aus der Tragödie, albern in Wortspielen, [Prevarication juxen] sich durch Schillerbrocken und Kantinenwitze, mal mühsam, mal spaßig, immer bemüht - und manchmal führt das Bemühen zu Erfolgen, und man sieht ein, dass alle politischen Ränke nur von der Gier „auf irgendwas“ angetrieben und nur durch Gehirnleere der Menschen ermöglicht werden. (Die Zeit, 27.04.2000, Nr. 18)

(77) Ich [Hostile_encounter kämpfte] mich durch dieses Unterholz, hielt mich an den Stämmen, Asten und Wurzeln fest, weil ich hinauf zum Gipfel wollte, der wie jeden Tag in den Wolken lag; wie dick die Schicht war, ließ sich nicht sagen - vielleicht schien oben die Sonne. (Schrott, Raoul: Tristan da Cunha oder die Hälfte der Erde; Hanser Verlag 2003, S. 161)

(78) Der Historiker [Perception_experience hört] sich in die Sprache ein, in der sich eine Klasse von Menschen zu orientieren versucht, er tritt in den Zeithorizont ihrer Voraussetzungen und Erwartungen ein und hat, so an den Begrenzungen teil, denen sie selbst ausgesetzt war. (Schlögel, Karl: Petersburg, München Wien: Carl Hanser Verlag 2002, S. 90)

Um den Grad dieser ,Kreativität‘ oder umgekehrt den Grad der Prototypikalität von Konstrukten, die wesentlich ,gewöhnlicher‘ als die Belege (76)-(78) erscheinen, zu messen, muss die Frage nach der (Un-)Verbundenheit zwischen

\footnotetext{
57 Unter dem Begriff der Kreativität fasst schon Langacker (1987: 71) sowohl die hier gemeinte produktive Instanziierung einer Konstruktion (mit einem unrelatierten lexikalischen Frame) als auch den eher generativistisch anmutenden Mechanismus zur Anwendung von Regeln bei der Produktion neuer Äußerungen zusammen - und verortet sie auf einem Kontinuum. Sampson (2016: 17) bezeichnet diese Form von Kreativität als F-Kreativität, wobei das F für fixiert steht. Hoffmann (2018: 262) und Bergs (2018: 278) bringen diese Form von Kreativität mit Produktivität in Verbindung. Kreativität in diesem Sinne scheint auch dem Verständnis von Goldberg (2019: 51-73) zugrunde zu liegen. Auf die sich durch den Prozess der Frame-Anpassung äußernde ,Kreativität‘ weist, ähnlich wie Herbst (2018a: 320) für den Begriff des Blendings im Sinne der Theorie der konzeptuellen Integration, auch Busse (2012: 674-675) hin.
} 
lexikalischem Frame und Konstruktions-Frame objektiviert werden. Als ein semantischer Parameter von Konstruktionen und ein Indiz für die Leistung des Konstruktions-Frames bei der Konstitution eines Konstrukt-Frames möchte ich deshalb die wortwörtliche Nähe bzw. Distanz zwischen jenen Frames heranziehen. Messen lässt sich diese auf der Grundlage von FrameNet-Daten mit Hilfe der Frame-zu-Frame-Relationen zwischen einem Konstruktions-Frame und möglichen lexikalischen Frames. Für den Parameter, der sich diese Methode zunutze macht, möchte ich den Terminus Frame-Nähe verwenden, angelehnt an den Begriff der Frame-Distanz, den Čulo (2013) für übersetzungswissenschaftliche Fragestellungen nutzt. ${ }^{58}$ Čulo (2013: 162) diskutiert die Idee, dass sich die semantische Verbundenheit von Übersetzungen messen lässt, indem man den Frame, der in einem Satz der Ausgangssprache evoziert wird, mit demjenigen, der in der Übersetzung des Satzes in der Zielsprache evoziert wird, vergleicht. Je weiter diese beiden Frames hinsichtlich ihrer Frame-zu-Frame-Relationen auseinander liegen, desto geringer die semantische Verbundenheit beider Sätze.

Unter dem (missverständlichen) Begriff der Frame-Valenz diskutieren Uchida \& Fujii (2011) eine ganz ähnliche Idee. Werden in einem Satz durch den Gebrauch eines Konnektors wie während zwei Frames evoziert, so lassen sich Verbindungen zwischen diesen Frames über Frame-zu-Frame-Relationen erfassen:

Since frames are linked to each other via frame-to-frame relationships, it is possible to make generalizations about each frame valence. This makes it possible to summarize the patterns of frame valence; otherwise the extremely long list of valences is not useful. (Uchida \& Fujii 2011: 134)

Tatsächlich gibt es Evidenz dafür, dass zwei so miteinander verbundene Frames häufig in einer Frame-zu-Frame-Relation zueinander stehen: „In examples where different frames are evoked in the two clauses, the frames are actually linked via higher frames in many cases.“ (Uchida \& Fujii 2011: 147). Frame-zuFrame-Relationen stellen demnach ein leistungsstarkes Instrument zur Messung der Distanz zwischen zwei Frames dar, einem lexikalischen Frame und dem Konstruktions-Frame. ${ }^{59}$

58 Auf den Begriff der Distanz verweist - eher informell - in Fragen von Kreativität auch Herbst (2018a: 320): „Different degrees of creativity arise from the distance between the units that are being blended (which can be related to form, semantics or world-knowledge).“

59 Eine verwandte Idee findet sich in der Frame-Forschung unter dem Begriff BedeutungsAdjazenz bereits bei Ballmer \& Brennenstuhl (1981: 303-306), die damit die semantische Zusammengehörigkeit nicht nur von Wörtern, die einen Frame evozieren, sondern auch jene von Frames insgesamt erfassen möchten. Anknüpfend an diese Idee und auf der Grundlage der AssoziationsStudien von Bartlett (1932) entwirft Busse (2012: 636-638) den Begriff der Frame-Kontinguität, der 
Ich möchte in diesem Abschnitt zeigen, dass es der Begriff der Frame-Nähe ermöglicht, lexikalische Frames von einem Konstruktions-Frame zu unterscheiden und die ,Nähe‘ Ersterer zu Letzterem zu bestimmen. Die Frame-Nähe zwischen einem lexikalischen Frame und dem Konstruktions-Frame kann als Anzeichen für den Eingang eines von dem lexikalischen Frame unterschiedenen KonstruktionsFrames in einen Konstrukt-Frame dienen und zugleich den graduellen Unterschied zwischen dem Konstruktions-Frame und möglichen lexikalischen Frames messen. So unterscheidet sich der Konstruktions-Frame am stärksten von einem lexikalischen Frame, wenn eine Frame-Nähe zwischen beiden durch Frame-zuFrame-Relationen in FrameNet nicht nachweisbar ist. Gleichzeitig gilt für diejenigen lexikalischen Frames, die mit dem Konstruktions-Frame durch Framezu-Frame-Relationen verbunden sind, dass sie sich umso deutlicher von ihm unterscheiden, je geringer die Frame-Nähe zwischen beiden Frames ist.

In Unterabschnitt 5.4.1 möchte ich das Grundprinzip der Frame-Nähe unter Rückgriff auf die Frame-zu-Frame-Relationen in FrameNet erläutern und eine Methode dafür vorstellen, die Menge der zu einem Konstruktions-Frame in FrameNähe stehenden lexikalischen Frames zu definieren. In Unterabschnitt 5.4.2 argumentiere ich dafür, das Konzept der Frame-Nähe prototypentheoretisch zu verstehen und die Frame-Nähe eines lexikalischen Frames in einem gegebenen Konstrukt als Indikator für die Prototypikalität dieses Konstrukts zu nutzen. Da für die weitere Analyse der drei untersuchten Konstruktionen die lexikalischen Frames, die zu ihrem Konstruktions-Frame Motion in Frame-Nähe stehen, von großer Relevanz sind, sei abschließend in Unterabschnitt 5.4.3 das vollständige System der Frame-Nähen von Motion, wie es für die drei Konstruktionen belegt ist, dargestellt.

\subsubsection{Frame-zu-Frame-Relationen und Frame-Nähe}

Um zu zeigen, wie die Frame-Nähen lexikalischer Frames zu einem gegebenen Konstruktions-Frame definiert sind, bietet es sich an, den Konstruktions-Frame der drei untersuchten Konstruktionen heranzuziehen. Am Beispiel von Motion möchte ich zeigen, wie ausgehend von den Frame-zu-Frame-Relationen, die für ihn in FrameNet 1.7 dokumentiert sind, diejenigen Frames ermittelt werden kön-

auf die assoziativen Relationen zwischen zwei Frames abzielt. In diesem Zusammenhang stellt Busse (2012: 643-644) sodann eine Typologie assoziativer Netzwerke auf, die solche Frames bilden können. Interessanterweise verweist er am Rande auf Konstruktionen im Sinne der Konstruktionsgrammatik, deren Strukturen sich mit Frame-Kookkurrenzen vergleichen lassen (vgl. Busse 2012: 644, Anm. 189). 
nen, die zu ihm in Frame-Nähe stehen. Abbildung 5.4 zeigt die Frames, mit denen Motion in der Vererbungsrelation verbunden ist. Diese Informationen sind über den FrameGrapher ${ }^{60}$ von FrameNet verfügbar, ich stelle allerdings lediglich alle unter- und übergeordneten Frames von Motion sowie zwei Frames, die auf derselben Hierarchieebene wie er liegen, dar.

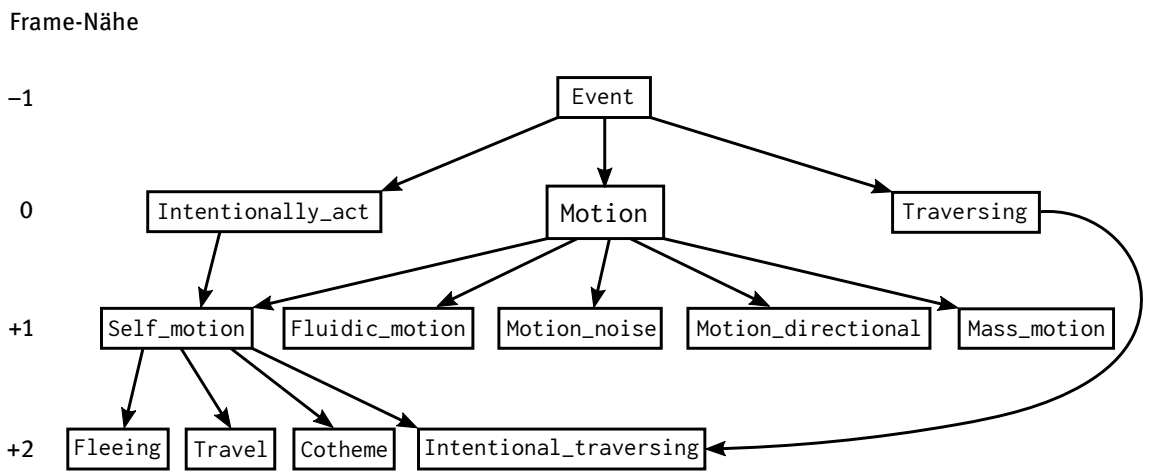

Abb. 5.4: Frames in Vererbungsrelation zu Motion hinsichtlich ihrer Frame-Nähen in FrameNet 1.7 (vereinfacht in Anlehnung an den FrameNet-FrameGrapher)

Frame-Nähen sind grundsätzlich in zwei Richtungen möglich: Sie verlaufen,nach unten' für all diejenigen Frames, die dem untersuchten Frame untergeordnet sind, und ,nach oben' für all diejenigen Frames, die dem untersuchten Frame übergeordnet sind. Für ersteren Fall liegt eine positive Frame-Nähe vor, der Abstand zum untersuchten Frame kann mit einem positiven Vorzeichen versehen werden. Für letzteren Fall liegt entsprechend eine negative Frame-Nähe vor, weshalb ein negatives Vorzeichen eingesetzt wird. Die Art des Vorzeichens (positiv oder negativ) zeigt somit lediglich die Richtung an, in der die Frame-zu-Frame-Relation verläuft. Je höher die darauffolgende Zahl ist, desto geringer die Frame-Nähe. Umgekehrt zeigt eine niedrige Zahl also keine niedrige Frame-Nähe an, sondern eine große.

Motion besitzt fünf Frames, die ihm in der Vererbungsrelation direkt untergeordnet sind: Fluidic_motion, Mass_motion, Motion_directional, Motion_noise

$60 \mathrm{https} / / /$ framenet.icsi.berkeley.edu/fndrupal/FrameGrapher (zuletzt abgerufen am 07.09. 2021). Die dem FrameGrapher zugrunde gelegten Frame-zu-Frame-Relationen beruhen auf dem auf der FrameNet-Website dokumentierten FrameNet-Release und nicht auf dem Release 1.7, das ich verwende. Ich lege aus Konsistenzgründen deshalb die Frame-zu-Frame-Relationen des Release 1.7 zugrunde, wenngleich sie sich von dem auf der Website dokumentierten Stand unterscheiden. 
und Self_motion. Sie alle stehen also in einer Frame-Nähe von +1 zu ihm. Self_motion wiederum steht als einziger dieser Frames zu Frames, die ihm direkt untergeordnet sind, in Vererbungsrelation: Cotheme, Fleeing, Intentional_traversing und Travel stehen zu Motion damit gewissermaßen indirekt in Verbindung: Sie erhalten in dieser Konstellation eine Frame-Nähe von +2 . Motion übergeordnet ist in der Vererbungsrelation nur ein Frame: Event. Er steht aus dieser Perspektive also zu ihm in einer Frame-Nähe von -1. Motion selbst erhält die Frame-Nähe 0. Voraussetzung dafür, dass Frame-Nähen von +2 und höher (sowie solche von -2 und niedriger, die für Motion aber nicht definiert sind) möglich sind, ist die Annahme einer mathematischen Transitivität der Hierarchie (vgl. dazu Stock \& Stock 2008: 70-72). Wenn also die Relation Self_motion ,ist ein' Motion gilt, ${ }^{61}$ gilt zugleich die Relation Fleeing, ist ein' Motion.

Als Frames, die in Frame-Nähe zu Motion liegen, betrachte ich allerdings nur diejenigen, die in einer gleichbleibenden Frame-zu-Frame-Relation (bei FrameNähen von +2 und höher) ${ }^{62}$ sowie über einen direkten Relationspfad auf Moti ion zurückzuführen sind, ohne die Richtung der Hierarchie zu wechseln. ${ }^{63}$ In Abbildung 5.4 zählen Intentionally_act und Traversing somit nicht dazu, wenngleich sie auf derselben Hierarchieebene wie Motion liegen. Ein Beispiel für die BenutztRelation mag dies weiter illustrieren: Der Frame Fastener ist Closure untergeordnet, welcher auf derselben Hierarchieebene wie Mot ion liegt. Motion und Closure sind allerdings nicht relational miteinander verbunden, ein gemeinsamer übergeordneter Frame etwa fehlt. Die Verbindung besteht lediglich darin, dass der Frame Clothing, der Closure in einer Benutzt-Relation untergeordnet ist, den Frames Dressing und Undressing übergeordnet ist, die zu Motion in einer Frame-

61 Vgl. dazu, dass die Vererbungsrelation als „,corresponding to is-a in many ontologies“ (Ruppenhofer et al. 2016: 80) aufgefasst wird.

62 Ein Wechsel der Relationen würde den Grundsatz der Transitivität gefährden, vgl. dazu das anschauliche Beispiel bei Stock \& Stock (2008: 71): „Nehmen wir z.B. an, die Leber von Professor $\mathrm{X}$ ist Teil von $\mathrm{X}$ und Professor $\mathrm{X}$ ist Teil der Universität $\mathrm{Y}$, dann gilt bei Transitivität: Die Leber von Professor X ist Teil der Universität Y, was offensichtlich Unfug ist. Aber Achtung! War das überhaupt dieselbe Relation? Die Leber ist ein Körperteil; ein Professor ist ein Teil einer Organisation. Nur weil wir vereinfachend von einer allgemeinen Ganzes-Teil-Relation ausgehen, gilt die Transitivität nicht.“

63 Für das in dieser Hinsicht betrachtete Frame-Netzwerk gilt somit dasselbe wie für ein Konstruktionsnetzwerk: „Constructional characteristics, that is, characteristics of form and meaning, are inherited in a downwards direction, from higher, more schematic levels towards lower, more concrete levels.“ (Hilpert 2019: 58). Es gilt damit: „Inheritance is thus a ,downwards“ relation; more specific constructional characteristics are not projected ,upwards'““ (Hilpert 2019: 59). Diese Sicht lässt sich nicht nur auf die Vererbungsrelationen, sondern auch auf alle anderen Frame-zuFrame-Relationen übertragen. 
Nähe von +2 stehen. Abbildung 5.5 zeigt diese Zusammenhänge durch einen Auszug aus den Benutzt-Relationen von Motion. Um von Fastener zu Motion zu gelangen, muss also dreimal die Richtung der Hierarchie gewechselt werden, da Dressing und Undressing dem Frame Placing untergeordnet sind, der wiederum erst Motion untergeordnet ist.

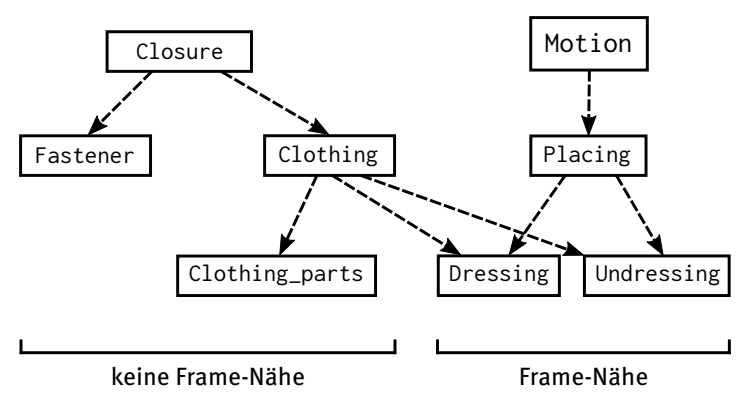

Abb. 5.5: Ausschnitt der Frames in Benutzt-Relation zu Motion in FrameNet 1.7 (vereinfacht in Anlehnung an den FrameNet-FrameGrapher)

Aus diesem Grund betrachte ich einen Frame wie Fastener nicht als in FrameNähe zu Motion stehend, ebenso wie alle anderen Frames, die auf der linken Seite in Abbildung $5.5 \mathrm{zu}$ sehen sind und nur durch einen Richtungswechsel in der Frame-Hierarchie zu erreichen wären (z.B. Clothing_parts). Gleiches gilt für die Frames Intentionally_act und Traversing in Abbildung 5.4, die ebenfalls nur durch einen Wechsel der Hierarchierichtung, hier innerhalb der Vererbungsrelation, erreichbar wären. Damit ist selbstverständlich nicht gesagt, dass diese Frames nicht als lexikalische Frames für die Konstitution von Konstrukt-Frames infrage kommen können. Tatsächlich machen solche lexikalischen Frames einen großen Anteil der Konstrukt-Frames der reflexiven Bewegungskonstruktion und der reflexiven Partikelverbkonstruktion aus.

\subsubsection{Indikatoren für die Prototypikalität eines Konstrukts}

Das System der Frame-Nähen eines Konstruktions-Frames lässt sich über die Feststellung des Inventars an lexikalischen Frames, die zu ihm in Frame-zu-FrameRelationen - auch über mehrere Hierarchieebenen hinweg - stehen, hinaus in der Analyse von Konstrukten und Konstrukt-Frames verwenden. In Anlehnung an die Prototypentheorie von Rosch (1978: 30), die eine vertikale und eine horizontale Dimension von Kategorien unterscheidet, können Frame-Nähen entlang 
ebendieser Dimensionen als Maßstab für die Prototypikalität von Konstrukten herangezogen werden. ${ }^{64} \mathrm{Da}$ es die lexikalischen Frames sind, die diese bestimmen, lässt sich schließlich zugleich diesen Frames eine entsprechende Prototypikalität oder Unprototypikalität zusprechen. Es ist davon auszugehen, dass Konstrukte, deren relatierte lexikalische Frames näher am Konstruktions-Frame liegen, als prototypischere Instanzen der Konstruktion zu betrachten sind als solche, in denen der lexikalische Frame in einer geringeren Frame-Nähe zum KonstruktionsFrame steht. Lexikalische Frames lassen sich also in zwei Klassen einteilen, auf die ich im weiteren Verlauf dieser Arbeit immer wieder zurückkommen werde.

a) Relatierte lexikalische Frames sind solche, die zum Konstruktions-Frame in einer (beliebig großen) Frame-Nähe stehen, einschließlich desjenigen lexikalischen Frames, der mit dem Konstruktions-Frame identisch ist.

b) Unrelatierte lexikalische Frames sind solche, die zum Konstruktions-Frame in keiner Frame-Nähe stehen.

Betrachtet man die Konstrukte einer Konstruktion nun als eine Kategorie, so strukturieren die Frame-Nähen der lexikalischen Frames der Konstrukte diese Kategorie hinsichtlich der Prototypikalität der Konstrukte. Diese interne Strukturierung der Kategorie ,Konstrukt der Konstruktion X' ist somit Ausdruck einer horizontalen prototypischen Dimension (vgl. dazu Rosch 1978: 30). Der KonstruktionsFrame stellt damit gewissermaßen die ,Kernbedeutung (core meaning) im Sinne von Rosch (1973: 140-141) dar. Konstrukte, deren lexikalische Frames direkt dem Konstruktions-Frame entsprechen (also eine Frame-Nähe von 0 aufweisen), sind die Prototypen der Kategorie. Je größer die Frame-Nähe eines relatierten lexikalischen Frames eines Konstrukts zum Konstruktions-Frame ist, desto prototypischer ist dieses Konstrukt für die Konstruktion. Lexikalische Frames mit einer geringen Frame-Nähe sind weiter vom prototypischen Kern, dem KonstruktionsFrame, entfernt, als solche mit einer weniger geringen Frame-Nähe. ${ }^{65}$ Die Kategorie der relatierten lexikalischen Frames im Sinne von a ist also in sich nach Prototypikalität strukturiert, die mit abnehmender Frame-Nähe abnimmt. Abbildung 5.6 veranschaulicht in Anlehnung an die Darstellungsform horizontaler prototypischer Dimensionen wie bei Aitchison (2012: 69) diese Zusammenhänge exempla-

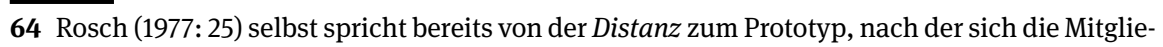
der einer Kategorie in ihrer Prototypikalität unterscheiden.

65 Auch Busse (2012: 598) weist im Zuge seiner Überlegungen zu Prototypikalität von Frames auf drei hier relevante Typen von Prototypikalität hin, die die Relationen zwischen Frames und die Konstitution ganzer Frame-Netzwerke betreffen, ohne sie jedoch weiter zu elaborieren. Der Begriff der Frame-Nähe als Indikator für Prototypikalität kann deshalb als eine mögliche Operationalisierung von Busses Thesen gesehen werden. 
risch für einige der positiven Frame-Nähen (+1 oder höher) der Vererbungsrelation von Motion. ${ }^{66}$

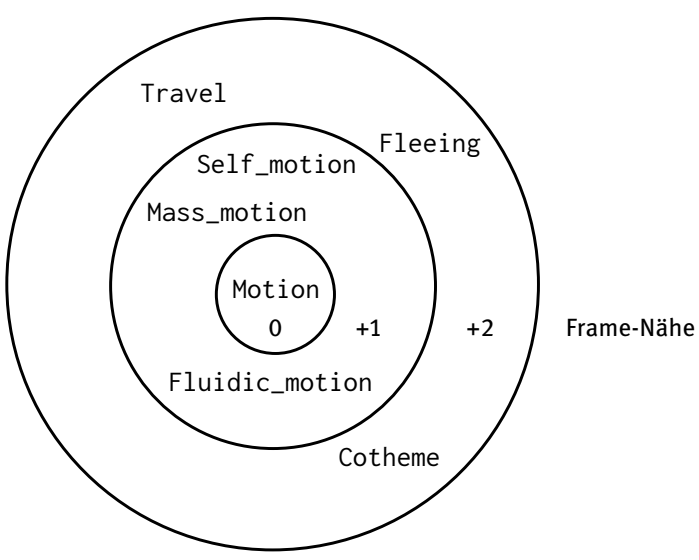

Abb. 5.6: Positive Frame-Nähen (Auswahl) zur Strukturierung einer prototypischen Kategorie relatierter Frames, am Beispiel der Vererbungsrelation von Motion in FrameNet 1.7

Die Prototypikalität eines Konstrukts, wie sie über die Frame-Nähe des lexikalischen Frames bestimmt werden kann, trifft allerdings keine Aussagen über die Gesamtheit der Konstrukte, die als Instanzen der betreffenden Konstruktion angesehen werden können. Hinzu kommen noch all diejenigen Konstrukte, deren lexikalische Frames nicht in einer Frame-zu-Frame-Relation zum KonstruktionsFrame stehen und für die sich somit keine Frame-Nähe ermitteln lässt - mit anderen Worten: die unrelatiert sind. Auf diesen Aspekt komme ich im nächsten Unterabschnitt 5.4.3 zurück. Wie bereits erwähnt, decken die Konstrukte, deren Konstrukt-Frames einen lexikalischen Frame in Frame-zu-Frame-Relation zum Konstruktions-Frame aufweisen, jedoch bereits einen Teil der Konstrukte ab. ${ }^{67}$ Lexikalische Frames in Frame-Nähe zum Konstruktions-Frame sind mit der Ausprägung ihrer Frame-Nähen nicht nur ein Indikator für die Prototypikalität eines

66 Dahingehend, dass der Übergang zwischen relatierten und unrelatierten lexikalischen Frames graduell ist, lässt sich auch die Annahme eines Kontinuums zwischen Elaboration und Fusion bei Hampe \& Schönefeld (2003: 248) deuten (vgl. auch Unterabschnitt 7.4.1). Zum Begriff der Elaboration vgl. Unterabschnitt 6.2.1.

67 Es ist somit nicht nötig, wie Mortelmans \& Smirnova (2020: 61-62) es tun, inhärente (reflexive) Bewegungsverben von Konstrukten der reflexiven Bewegungskonstruktion zu trennen. Instanzen mit Ersteren sind schlicht als verschieden prototypische Konstrukte (mit relatierten lexikalischen Frames) zu verstehen, Instanzen mit Letzteren als solche mit unrelatierten lexikalischen Frames. 
Konstrukts, sondern zugleich ein Indikator dafür, ob ein Beleg ein Konstrukt der betrachteten Konstruktion enthält (dazu Unterabschnitt 8.4.2). ${ }^{68}$ Alle Instanzen, deren lexikalische Frames in einer (direkten oder indirekten) Frame-zu-FrameRelation zum Konstruktions-Frame stehen, gleich welcher Frame-Nähe, kommen somit als Konstrukte der betreffenden Konstruktion infrage. Dies erleichtert die Datenauswahl erheblich und erklärt die Anzahlen der relevanten Konstrukte für die drei untersuchten Konstruktionen, wie sie in Abschnitt 3.4.1 aufgelistet sind.

Die Prototypikalität von Konstrukten, deren lexikalische Frames in FrameNähe zum Konstruktions-Frame stehen, wirkt sich nun auf mindestens die folgenden Aspekte aus, von denen die ersten beiden zugleich den Zusammenhang dieses semantischen Parameters von Konstruktionen zu anderen Parametern ausdrücken.

- Die Entstehung von Koerzionseffekten: Konstrukt-Frames mit relatierten lexikalischen Frames weisen keine Koerzionseffekte (Abschnitte 5.5 und 7.4) auf. Dies schon deshalb nicht, weil bei ihnen kein zusätzlicher KonstruktionsFrame an der Konstitution des Konstrukt-Frames beteiligt ist, also keine Frame-Anpassung stattfindet. Steht der lexikalische Frame aber nicht in Frame-Nähe zum Konstruktions-Frame, ist Letzterer automatisch beteiligt und konstituiert gemeinsam mit dem lexikalischen Frame den KonstruktFrame. Da der lexikalische Frame nun nicht mehr alle KtE allein motiviert, ergeben sich Koerzionseffekte. ${ }^{69}$

- Die Produktivität der Konstruktion: Eine Konstruktion ist dann besonders produktiv, wenn besonders viele unterschiedliche unrelatierte lexikalische Frames in die Konstitution ihrer Konstrukt-Frames eingehen, die Type-Frequenz der unrelatierten lexikalischen Frames also besonders hoch ist (vgl. Unterabschnitt 5.6.2 sowie Abschnitt 7.5). Die Untersuchung von Frame-Nähen ist damit eine wesentliche Voraussetzung der Untersuchung der Produktivität einer Konstruktion.

- Die semantische Motivierung von KtE und KEE (Abschnitte 6.2 und 6.3): Wird ein Frame als lexikalischer Frame eingesetzt, der in Frame-Nähe zum Konstruktions-Frame steht, so werden alle KtE des Konstrukts durch den lexikalische Frame motiviert (vgl. Unterabschnitt 6.2.1). Ein separater KonstruktionsFrame ist in diesen Fällen nicht an der Konstitution des Konstrukt-Frames beteiligt.

68 Auf die schon mehrfach erwähnte Ausnahme für die reflexive Partikelverbkonstruktion komme ich in Unterabschnitt 6.4.3 zu sprechen.

69 Auf die Messung des Koerzionspotenzials einer Konstruktion aufgrund dieser Verhältnisse in der Konstitution des Konstrukt-Frames gehe ich in den Unterabschnitten 7.4.1 und 7.4.3 ein. 
- Die Evokation des Konstruktions-Frames (Unterabschnitt 8.1.2 sowie Abschnitte 8.2 und 8.3): Ob in einem Konstrukt ein relatierter oder unrelatierter lexikalischer Frame vorliegt, entscheidet wesentlich über den Mechanismus zur Evokation des Konstruktions-Frames. In methodischer Hinsicht kann zudem das System eines potenziellen Konstruktions-Frames herangezogen werden, um zu überprüfen, ob dieser Frame tatsächlich als Konstruktions-Frame infrage kommt (vgl. Unterabschnitt 8.5.1).

Beide Arten von Konstrukten, solche mit relatierten wie solche mit unrelatierten lexikalischen Frames, kommen als Instanzen der Konstruktion infrage. Ist der Konstruktions-Frame an der Konstitution des Konstrukt-Frames beteiligt, steht dies ohnehin außer Frage. Ein Konstrukt, dessen KtE durch FE eines lexikalischen Frames vollständig motiviert werden (Unterabschnitt 6.2.1), kommt als Instanz der betreffenden Konstruktion nur dann infrage, wenn der lexikalische Frame in Frame-Nähe zum Konstruktions-Frame steht. Ist dies nicht der Fall, handelt es sich in der Regel nicht um eine Instanz der betreffenden Konstruktion (vgl. aber Unterabschnitt 6.4.3 für ein Gegenbeispiel bei der reflexiven Partikelverbkonstruktion). Ein Blick auf die Frame-Nähe eines lexikalischen Frames ist somit ein wichtiges Hilfsmittel bei der Frage danach, ob ein gegebenes Konstrukt als Instanz einer zu untersuchenden Konstruktion gelten kann oder nicht. ${ }^{70}$

\subsubsection{Frame-Nähen von Motion}

Als Basis für alle weiteren konstruktionssemantischen Analysen der drei untersuchten Konstruktionen möchte ich in diesem Unterabschnitt das vollständige System der Frame-Nähen von Motion aufzeigen. Für die Vererbungsrelation habe ich damit in Unterabschnitt 5.4 .1 bereits begonnen: Abbildung 5.4 zeigt alle Frames, die innerhalb der Vererbungsrelation in Frame-Nähe zu Motion stehen. Alternativ zu der graphischen Darstellung lassen sich diese Zusammenhänge auch tabellarisch darstellen. Tabelle 5.12 zeigt eine Auflistung der relevanten Frames und deren Zuordnung zu den unterschiedlichen Frame-Nähen. Die Zuordnung über- und untergeordneter Frames zueinander spielt hierbei keine Rolle: So ist aus der Tabelle nicht ersichtlich, dass die Frames Cotheme, Fleeing, Intentional_traversing und Travel gerade Self_motion untergeordnet sind und nicht etwa Motion_directional. Da diese Details für die folgenden Analy-

70 Auf den damit verbundenen methodologischen Aspekt hinsichtlich der Disambiguierung von Konstruktionen mit einer ambigen Formseite gehe ich in Unterabschnitt 8.4.2 ein. 
sen nicht von Relevanz sind, ignoriere ich das Problem, sie nicht darstellen zu können, zugunsten einer übersichtlicheren Darstellung in Tabellenform.

Tab. 5.12: Frame-Nähen der Vererbungsrelation von Motion nach FrameNet 1.7

\begin{tabular}{rlrrr}
\hline Frame-Nähe & Frame & RBKxn & RPVKxn & Weg-Kxn \\
\hline-1 & Event & 0 & 0 & 0 \\
0 & Motion & 105 & 24 & 26 \\
+1 & Fluidic_motion & 17 & 0 & 0 \\
& Mass_motion & 2 & 0 & 0 \\
& Motion_directional & 11 & 15 & 0 \\
& Motion_noise & 0 & 0 & 0 \\
& Self_motion & 159 & 56 & 0 \\
& Cotheme & 0 & 0 & 0 \\
& Fleeing & 5 & 0 & 0 \\
& Intentionally_traversing & 0 & 0 & 0 \\
& Travel & 0 & 0 & 0 \\
\hline
\end{tabular}

Um die Verteilung relatierter lexikalischer Frames, die in den Konstrukten der drei untersuchten Konstruktionen evoziert werden können, hinsichtlich ihrer FrameNähe zu illustrieren, sind in allen Tabellen in diesem Unterabschnitt die TokenFrequenzen der entsprechenden lexikalischen Frames, die auf die reflexive Bewegungskonstruktion (RBKxn), die reflexive Partikelverbkonstruktion (RPVKxn) sowie die reflexive $\mathrm{Weg}$-Konstruktion (Weg-Kxn) entfallen, angegeben. Die lexikalischen Frames in Frame-Nähe zu Motion gehe ich für jede einzelne der im FrameNet dokumentierten Frame-zu-Frame-Relationen, die ich in Unterabschnitt 2.1.2 aufgezählt habe, durch. Da ich dort noch nicht auf die Definitionen der Relationen eingegangen bin, soll dies an dieser Stelle nachgeholt werden.

Die wichtigste und zuerst zu betrachtende Frame-zu-Frame-Relation ist die Vererbungsrelation. Sie ist ,the strongest relation between frames, corresponding to is-a in many ontologies“ (Ruppenhofer et al. 2016: 80). Sie führt dazu, dass der jeweils untergeordnete Frame über alle Eigenschaften des übergeordneten Frames verfügt, entweder in derselben oder einer höheren Spezifiziertheit (vgl. Ruppenhofer et al. 2016: 80). Wie aufgrund ihres Status als wichtigste Framezu-Frame-Relation zu erwarten, stellt die Vererbungsrelation eine der quantitativ größten Frame-zu-Frame-Relationen dar, deren Frames als lexikalische Frames in Konstrukt-Frames der drei untersuchten Konstruktionen eingehen. Wie aus Tabelle 5.12 hervorgeht, sind für die reflexive Bewegungskonstruktion unter den lexikalischen Frames mit Frame-Nähe von +1 in der Summe mehr Konstrukte 
als mit dem lexikalischen Frame Motion, also einer Frame-Nähe von 0, belegt. Gleiches gilt für die reflexive Partikelverbkonstruktion.

Frame-Nähen lassen sich nicht nur für die Vererbungsrelation feststellen, sondern für alle Frame-zu-Frame-Relationen. Eine Relation, die in Bezug auf Motion als Konstruktions-Frame ein durchaus komplexes System von Frame-Nähen aufweist, ist die Benutzt-Relation. Sie ist „one of the largest frame-to-frame relations in FrameNet“ (Sikos \& Padó 2018: 43) und sagt aus, dass ,a particular frame makes reference in a very general kind of way to the structure of a more abstract, schematic frame.“ (Ruppenhofer et al. 2016: 83). Dieser Bezug zu einem übergeordneten Frame gilt ,almost exclusively for cases in which a part of the scene evoked by the child [dem untergeordneten Frame, A.W.] refers to the parent frame [dem übergeordneten Frame, A.W.]“ (Ruppenhofer et al. 2016: 83). ${ }^{71}$

Anders als die Vererbungsrelation weist die Benutzt-Relation Frame-Nähen von bis $\mathrm{zu}+3$ auf, während eine Frame-Nähe von -1 oder niedriger nicht existiert. Die Benutzt-Relation beinhaltet für die reflexive Bewegungskonstruktion die meisten Konstrukte, die in Frame-Nähe zum Konstruktions-Frame stehen, noch vor der Vererbungsrelation. Besonders frequent ist der lexikalische Frame Body_movement. Für die reflexive Partikelverbkonstruktion ist er ebenso der frequenteste aller lexikalischen Frames in dieser Frame-zu-Frame-Relation (vgl. Tabelle 5.13).

Über lediglich eine Stufe der Frame-Nähe verfügt die Relation Sub-Frame. Sie umfasst untergeordnete Frames, die Teilhandlungen des übergeordneten Frames darstellen und in einer (i.d.R. zeitlichen) Abfolge zueinander stehen:

\begin{abstract}
Some frames are complex in that they refer to sequences of states and transitions, each of which can itself be separately described as a frame. The separate frames (called subframes) are related to the complex frames via the SubFrame relation. In such cases, frame elements of the complex frame may be connected to the frame elements of the subparts, although not all frame elements of one need have any relation to the other. [...] Also, the ordering and other temporal relationships of the subframes can be specified using binary precedence relations. (Ruppenhofer et al. 2016: 83)
\end{abstract}

Die Sub-Frame-Relation weist, wie Tabelle 5.14 zeigt, nur zwei Frames in einer Frame-Nähe von +1 auf. Für keine der von mir untersuchten Konstruktionen sind

71 Sikos \& Padó (2018: 43) weisen darauf hin, dass noch eine zweite Definition der BenutztRelation bei Petruck \& de Melo (2012: 46) existiert und dass nicht alle Frames, die über diese Relation miteinander verbunden sind, mit einer einzigen Definition zu erfassen sind. Da es mir nicht auf eine eingehendere Diskussion dieser Relation ankommt, sehe ich über diese Probleme hinweg und orientiere mich, wie für die anderen Relationen auch, an der Definition von Ruppenhofer et al. (2016). 
Tab. 5.13: Frame-Nähen der Benutzt-Relation von Motion nach FrameNet 1.7

\begin{tabular}{|c|c|c|c|c|}
\hline Frame-Nähe & Frame & RBKxn & RPVKxn & Weg-Kxn \\
\hline 0 & Motion & 105 & 24 & 26 \\
\hline \multirow[t]{15}{*}{+1} & Body_movement & 246 & 118 & 0 \\
\hline & Bringing & 17 & 8 & 0 \\
\hline & Change_direction & 43 & 17 & 0 \\
\hline & Cotheme & 0 & 0 & 0 \\
\hline & Departing & 6 & 0 & 0 \\
\hline & Emanating & 0 & 0 & 0 \\
\hline & Evading & 7 & 0 & 0 \\
\hline & Excreting & 0 & 1 & 0 \\
\hline & Light_movement & 0 & 0 & 0 \\
\hline & Operate_vehicle & 0 & 1 & 0 \\
\hline & Path_traveled & 0 & 0 & 0 \\
\hline & Placing & 14 & 11 & 0 \\
\hline & Redirecting & 0 & 0 & 0 \\
\hline & Removing & 0 & 0 & 0 \\
\hline & Roadways & 0 & 0 & 0 \\
\hline \multirow[t]{12}{*}{+2} & Arranging & 0 & 0 & 0 \\
\hline & Convoy & 0 & 0 & 0 \\
\hline & Disembarking & 0 & 0 & 0 \\
\hline & Dressing & 0 & 0 & 0 \\
\hline & Dunking & 0 & 0 & 0 \\
\hline & Entourage & 0 & 0 & 0 \\
\hline & Facial_expression & 0 & 0 & 0 \\
\hline & Installing & 0 & 0 & 0 \\
\hline & Piracy & 0 & 0 & 0 \\
\hline & Sending & 0 & 0 & 0 \\
\hline & Storing & 0 & 0 & 0 \\
\hline & Undressing & 1 & 0 & 0 \\
\hline \multirow[t]{5}{*}{+3} & Carry_goods & 0 & 0 & 0 \\
\hline & Delivery & 0 & 0 & 0 \\
\hline & Making_faces & 1 & 0 & 0 \\
\hline & Sent_items & 0 & 0 & 0 \\
\hline & Store & 0 & 0 & 0 \\
\hline
\end{tabular}

Tab. 5.14: Frame-Nähen der Sub-Frame-Relation von Motion nach FrameNet 1.7

\begin{tabular}{rlrrr}
\hline Frame-Nähe & Frame & RBKxn & RPVKxn & Weg-Kxn \\
\hline 0 & Motion & 105 & 24 & 26 \\
+1 & Getting_underway & 0 & 0 & 0 \\
& Halt & 0 & 0 & 0 \\
\hline
\end{tabular}


Tab. 5.15: Frame-Nähen der Perspektive_auf-Relation von Motion nach FrameNet 1.7

\begin{tabular}{rlrrr}
\hline Frame-Nähe & Frame & RBKxn & RPVKxn & Weg-Kxn \\
\hline-1 & Motion_scenario & 0 & 0 & 0 \\
0 & Motion & 105 & 24 & 26 \\
\hline
\end{tabular}

Tab. 5.16: Frame-Nähen der Kausativ-Relation von Motion nach FrameNet 1.7

\begin{tabular}{rlrrr}
\hline Frame-Nähe & Frame & RBKxn & RPVKxn & Weg-Kxn \\
\hline-1 & Cause_motion & 77 & 53 & 0 \\
0 & Motion & 105 & 24 & 26 \\
\hline
\end{tabular}

Konstrukte mit lexikalischen Frames belegt, die in einer Sub-Frame-Relation zu Motion stehen.

Eine Frame-zu-Frame-Relation, die ausschließlich eine negative und keine positive Frame-Nähe konstituiert, ist Perspektive_auf. Voraussetzung für diese Relation ist „the presence of at least two different possible points-of-view on the Neutral [sic!] frame“ (Ruppenhofer et al. 2016: 82). Die Perspektive_auf-Relation beinhaltet, wie in Tabelle 5.15 dargestellt, einen Frame in einer Frame-Nähe von -1. Der Motion übergeordnete Frame Motion_scenario stellt hierbei den neutralen Frame dar, während Motion eine Perspektive auf diesen darstellt. Ebenso wie für die Sub-Frame-Relation sind für die drei untersuchten Konstruktionen für die Frame-zu-Frame-Relation Perspektive_auf keine Konstrukte belegt.

Eine Frame-zu-Frame-Relation, die wie die Perspektive_auf-Relation allein über einen Motion übergeordneten Frame, aber keine untergeordneten Frames verfügt und somit allein eine Frame-Nähe von -1 konstituiert, stellt die KausativRelation dar. Sie wird in der FrameNet-Dokumentation von Ruppenhofer et al. (2016: 85), wie für andere Schriften von FrameNet schon Busse (2012: 190) kritisiert, „nicht näher definiert, sondern nur an Beispielen erläutert“, was insbesondere für die Darstellung von Petruck et al. (2004) gilt, in der sie zusammen mit der Inchoativ-Relation eingeführt wird. Dennoch wirft sie einige, insbesondere im Sprachvergleich zwischen den für das Englische konzipierten FrameNet-Frames und deutschen Reflexivkonstruktionen, relevante Probleme auf, auf die ich in Unterabschnitt 6.3.1 zurückkomme. Auch die Kausativ-Relation ordnet Motion lediglich einen Frame in Frame-Nähe von -1 über: Cause_motion. Sie ist in Tabelle 5.16 zusammengefasst.

Ein wiederum komplexeres System von Frame-Nähen liegt bei der Siehe_auchRelation vor, der letzten, für die überhaupt Frame-Nähen von Motion ermittelt werden können. Vorbehalten ist sie „groups of frames that are similar and should 
Tab. 5.17: Frame-Nähen der Siehe_auch-Relation von Motion nach FrameNet 1.7

\begin{tabular}{rlrrr}
\hline Frame-Nähe & Frame & RBKxn & RPVKxn & Weg-Kxn \\
\hline 0 & Motion & 105 & 24 & 26 \\
+1 & Bringing & 17 & 8 & 0 \\
& Operate_vehicle & 0 & 1 & 0 \\
& Ride_vehicle & 1 & 0 & 0 \\
& Self_motion & 159 & 56 & 0 \\
+2 & Passing & 0 & 0 & 0 \\
& Sending & 0 & 0 & 0 \\
& Spatial_contact & 0 & 0 & 0 \\
+3 & Topic & 0 & 0 & 0 \\
& Wearing & 0 & 0 & 0 \\
\hline
\end{tabular}

be carefully differentiated, compared, and constrasted“ (Ruppenhofer et al. 2016: 85). Sie kann als die schwächste aller Frame-zu-Frame-Relationen gelten: „Due to its less structured semantic nature, the See_also relation freely violates any and all limitations normally expected for frame-to-frame relations“ (Ruppenhofer et al. 2016: 85). Wie Tabelle 5.17 zeigt, liegen für die Siehe_auch-Relation Frame-Nähen bis $\mathrm{zu}+3$ vor.

Für die verbleibenden Relationen Inchoativ und Vorausgehend sind in FrameNet 1.7 keine Frame-zu-Frame-Relationen zu Moti on dokumentiert und somit auch keine Frame-Nähen ermittelbar. Deshalb verzichte ich darauf, sie zu definieren.

Eine Gemeinsamkeit, die sich für alle Relationen und die Frame-Nähen der Frames, die zu Motion in Verbindung stehen, zeigt, ist, dass die Anzahl der Konstrukte mit geringerer Frame-Nähe teils deutlich abnimmt. Für die reflexive Bewegungskonstruktion übersteigt die Frame-Nähe +1 für alle Frame-zu-FrameRelationen zusammengenommen mit kumuliert 699 Konstrukten Motion selbst (105 Konstrukte) um ein Vielfaches. ${ }^{72}$ Die Frame-Nähe von +2 allerdings beinhaltet nur noch sechs Konstrukte und für eine Frame-Nähe von +3 sind noch weniger Konstrukte belegt, genauer: nur ein einziges Konstrukt. Für die reflexive Partikelverbkonstruktion lassen sich noch deutlichere Beobachtungen machen: Hier sind nur Konstrukte bis zu einer Frame-Nähe von +1 belegt, auch diese überschreiten mit kumuliert 292 Token diejenigen mit dem Konstruktions-Frame (24 Konstrukte) um ein Vielfaches. Niedrigere Frame-Nähen (von +2 oder mehr) treten für diese Konstruktion nicht auf. Tabelle 5.18 zeigt zusammengefasst die Anzahl der Kon-

72 Da einige Frames innerhalb der unterschiedlichen Frame-zu-Frame-Relationen mehrfach erscheinen, sind diese in der Auflistung in Tabelle 5.18 entsprechend mehrfach enthalten. Deshalb sind keine Summen für die Konstrukte der drei Konstruktionen angegeben. 
Tab. 5.18: Konstrukte der untersuchten Konstruktionen in Frame-Nähe zum KonstruktionsFrame Motion

\begin{tabular}{rrrr}
\hline Frame-Nähe & RBKxn & RPVKxn & Weg-Kxn \\
\hline-1 & 77 & 53 & 0 \\
0 & 105 & 24 & 26 \\
+1 & 699 & 292 & 0 \\
+2 & 6 & 0 & 0 \\
+3 & 1 & 0 & 0 \\
\hline
\end{tabular}

strukte und deren Frame-Nähen für alle Frame-zu-Frame-Relationen. Diese Zusammenhänge lassen auf die abnehmende Prototypikalität dieser Konstrukte als Instanzen der Konstruktion schließen. Die mit abnehmender Frame-Nähe abnehmende Prototypikalität spiegelt sich in der abnehmenden Anzahl von belegten Konstrukten wider. ${ }^{73}$ Auf die Implikationen dieser stark sinkenden Frequenzen mit abnehmenden Frame-Nähen komme ich in Unterabschnitt 8.2.2 zurück.

Im Folgenden möchte ich einige der Frame-Nähen von Motion durch Beispiele für die reflexive Bewegungskonstruktion illustrieren. Ich beschränke mich hierfür auf die Vererbungsrelation und somit die in Tabelle 5.12 aufgelisteten lexikalischen Frames. Wird das KE EREIGNIS der jeweiligen Konstruktion durch LE instanziiert, die ebenfalls Mot i on evozieren, beträgt die Frame-Nähe 0, da KonstruktionsFrame und lexikalischer Frame identisch sind. Dies trifft am Beispiel der reflexiven Bewegungskonstruktion auf LE wie bewegen (move.v), schlängeln (snake.v) oder mäandern (meander.v) zu, wie die Belege in (79) zeigen.

a. Er [Motion bewegte] sich durch vermeintlich unauffällige harmonische Räume mit einer Sehnsucht nach Farbigkeit, die alle Grauwerte des Theoretischen löschte. (Die Zeit, 10.02.2000, Nr. 7)

b. Ich [Motion schlängelte] mich durch die Autos und die Abgase, die rot zwischen den Schlußlichtern dampften, und entdeckte gerade noch rechtzeitig die kräftige Gestalt von John Bird hinter dem erleuchteten

73 Allerdings zeigt McColm (2019: 109-116), dass zunehmend von der ,Konstruktionsbedeutung، abweichende lexikalische Bedeutungen diachron Evidenz für die Herausbildung der Konstruktion als eigenständige Einheit ist. Ähnliches stellen mit explizitem Bezug auf den Begriff der Distanz auch Gisborne \& Patten (2011: 99) fest: „In the case of the way-construction, verbs from similar semantic fields are accommodated to the construction first, with verbs from more distal semantic fields being added later." Im Sinne des Konzepts der Frame-Nähe lassen sich diese Beobachtungen tendenziell als eine Zunahme belegter unrelatierter lexikalischer Frames interpretieren. Auch dies spricht für die besondere ,Kreativität‘, die Konstrukten mit einem unrelatierten lexikalischen Frame zukommt. 
Schaufenster. (Franck, Julia: Lagerfeuer, Köln: DuMont Literatur und Kunst Verlag 2003, S. 256)

c. So [Motion mäandern] sich die nackten und verschlungenen Leiber durch die Werbung und über die Titel jener der Werbeästhetik folgenden Magazine. (Die Zeit, 13.01.2000, Nr. 3)

Eine durchaus große Anzahl von Konstrukten ist für lexikalische Frames belegt, die in einer Frame-Nähe von +1 zum Konstruktions-Frame stehen. In den Daten für die reflexive Bewegungskonstruktion sind unter anderem folgende Konstrukte für die lexikalischen Frames Self_motion (80), Fluidic_motion (81) und Mass_motion (82) belegt.

(80) Katharina [self_motion schlich] sich oben durch den Flur und kletterte die Leiter zum Dachboden hinauf. (Dölling, Beate: Hör auf zu trommeln, Herz, Weinheim: Beltz \& Gelberg 2003, S. 28)

(81) Sie hatte den Damm eines Speichersees durchbrochen, [Fluidic_motion ergoss] sich durch den Feldkanal in die Lapus, von der Lapus in die Somes und von der Somes jenseits der ungarischen Grenze in die Theiß. (Die Zeit, 24.02.2000, Nr. 9)

(82) Ich fühle mich doppelt wieder, und wärmeres Leben [Mass_motion ergießt] sich durch alle meine Nerven. (Safranski, Rüdiger: Friedrich Schiller, München Wien: Carl Hanser 2004, S. 164)

Für die reflexive Bewegungskonstruktion sind, wie erwähnt als einzige unter den drei untersuchten Konstruktionen, ebenso Konstrukte belegt, deren lexikalische Frames in einer Frame-Nähe von +2 zum Konstruktions-Frame stehen. Von den vier lexikalischen Frames, die dafür infrage kommen, ist lediglich Fleeing belegt, wie die Belege in (83) illustrieren.

(83) a. Die [Fleeing flüchtet] sich in eine Affäre mit ihrem Maklervorbild, dem noch größeren Fassadenkünstler Buddy Kane (Peter Gallagher). (Die Zeit, 20.01.2000, Nr. 4)

b. Nur wer nicht überzeugend politisch handeln kann, [Fleeing flüchtet] sich gern in die Welt der PR-Berater undWerbeagenturen. [sic!] (Tange, Ernst Günter: Zitatenschatz zur Politik, Frankfurt a. M.: Eichborn 2000, S. 84)

c. Panik und Niedergeschlagenheit wechselten auch bei ihr ab, sie [Fleeing flüchtete] sich zu ihrem chirurgischen Besteck, in ihre SchädelHirn-Traumata. (Stadler, Arnold: Sehnsucht, Köln: DuMont Literatur und Kunst Verlag 2002, S. 37) 
Nun sind allerdings nicht nur diejenigen Konstrukte mit lexikalischen Frames in Frame-Nähe zum Konstruktions-Frame interessant, sondern gerade die Konstrukte, in denen lexikalischer Frame und Konstruktions-Frame unrelatiert sind, stellen die als ,kreativ‘ einzustufenden Konstrukte dar. Wenn Michaelis (2015: 9) schreibt, dass „[c]ombining verb meaning and construction meaning requires interpreters to create a semantic link between the event denoted by the verb and that denoted by the construction“, so existiert diese semantische Verbindung nur bei solchen Konstrukten, deren lexikalischer Frame in einer Frame-Nähe zum Konstruktions-Frame steht. Eine semantische Verbindung zwischen lexikalischem Frame und Konstruktions-Frame ist somit ein hinreichendes, aber kein notwendiges Kriterium für die Konstitution eines Konstrukt-Frames eines Konstrukts, das als Instanz der betreffenden Konstruktion gelten kann.

Wie ich in Unterabschnitt 5.4.1 gezeigt habe, kommen nicht alle Frames, die über eine Relation erreichbar sind, als Frames in einer Frame-Nähe zu einem gegebenen Frame infrage, insbesondere solche nicht, die zu erreichen ein Wechsel in der Richtung der Frame-Hierarchie nötig ist. Deshalb ist davon auszugehen, dass eine Frame-Distanz nicht unendlich groß sein kann, denn ,[a]n obvious question is whether there is a fixed boundary beyond which one cannot speak of semantic relatedness anymore“ (Čulo 2013: 162). Damit kommt die Frage nach einer ungefähren Grenze der Prototypikalität von Konstrukten ins Spiel: „[I]t is not yet clear how many steps through the frame hierarchy we can take and still plausibly claim 'semantic similarity'.“ (Czulo 2017: 475). Der Wechsel in der Hierarchierichtung erscheint für eine solche Grenze praktikabel. Frame-Nähen können somit lediglich in eine Richtung verlaufen, entweder ,nach unten', wenn es sich um positive Frame-Nähen, also untergeordnete Frames, handelt, oder ,nach oben', wenn es sich um negative Frame-Nähen, also übergeordnete Frames, handelt.

Nichtsdestotrotz können, wie bereits erwähnt, auch unrelatierte Frames, die also nicht in einer Frame-Nähe zu einem Konstruktions-Frame wie Motion stehen, als lexikalische Frames in Konstrukt-Frames eingehen. Die drei im Eingang zu diesem Abschnitt zitierten Konstrukte in (76)-(78) zeigen solche unrelatierten lexikalischen Frames: Prevarication, Hostile_encounter und Perception_experience. Lexikalischer Frame und Konstruktions-Frame sind in solchen Fällen nicht über Frame-zu-Frame-Relationen miteinander verbunden, kurzum: Sie weisen keine Frame-Nähe zueinander auf, sodass diese lexikalischen Frames als unrelatiert einzustufen sind. Wenngleich in diesem Fall die Prototypikalität dieser Konstrukte nicht mehr anhand einer Frame-Nähe bestimmt werden kann, kann es sich dabei durchaus um markante Konstrukte handeln. Der Grund dafür liegt darin, dass solche Konstrukte gerade aufgrund der fehlenden Relation zwischen lexikalischem Frame und Konstruktions-Frame und der dadurch nicht vorhandenen Frame-Nähe auffallen. Diese Auffälligkeit basiert auf Koerzionseffekten, 
die entstehen, wenn lexikalischer Frame und Konstruktions-Frame gemeinsam einen Konstrukt-Frame konstituieren, wenn also eine Frame-Anpassung des lexikalischen Frames stattfindet. An dieser Stelle kommt der nächste semantische Parameter von Konstruktionen, das Koerzionspotenzial, ins Spiel.

\subsection{Koerzionspotenzial}

Unter dem Terminus Koerzion (coercion) ${ }^{74}$ ist in der Konstruktionsgrammatik das Phänomen bekannt, dass die semantischen Eigenschaften einer Konstruktion dazu in der Lage sind, diejenigen von LE zu ,überschreiben' (vgl. Michaelis 2003a, 2004, 2005). Liegt Koerzion vor, so übt - konstruktionssemantisch reformuliert - ein Konstruktions-Frame wesentlichen Einfluss auf die Konstitution eines Konstrukt-Frames aus, eben indem er einen lexikalischen Frame derart anpasst, dass die LE, die Letzterer evoziert, eine von ihrer üblichen lexikalischen Bedeutung abweichende Lesart erhält. Dies impliziert, dass lexikalische Frames bei der Konstitution von Konstrukt-Frames primär sind und der KonstruktionsFrame sekundär ist und dass dieses Verhältnis im Falle einer Koerzion zugunsten eines größeren Einflusses des Konstruktions-Frames verschoben wird (vgl. auch Panther \& Thornburg 1999). Gewissermaßen ist dies der Kern des von Michaelis (2004: 25) formulierten Override Principle: „If a lexical item is semantically incompatible with its morphosyntactic context, the meaning of the lexical item conforms to the meaning of the structure in which it is embedded." Tatsächlich besteht Koerzion in der Anpassung eines lexikalischen Frames, während der Konstruktions-Frame unverändert bleibt, wie Michaelis (2005: 61) feststellt: „As per the Override Principle, coercion is asymmetric: only the input type (the lexical expression), and not the output type (the construction's denotatum), is changed in the resolution of a type mismatch.“ Ich möchte im Folgenden dafür argumentieren, das Phänomen der Koerzion als einen wichtigen Indikator für die Rolle eines Konstruktions-Frames und dessen Einfluss auf mögliche Konstrukt-Frames zu verstehen.

Koerzionsphänomene eignen sich als Grundlage für einen semantischen Parameter für Konstruktionen, weil in Koerzionseffekten die Existenz eines Konstruktions-Frames und seine Beteiligung an der Konstitution von KonstruktFrames deutlich sichtbar wird. Voraussetzung dafür ist freilich ein deutlicher Unterschied zwischen lexikalischem Frame und Konstruktions-Frame - kurzum: eine nicht nachweisbare Frame-Nähe des Ersteren (Abschnitt 5.4) - und ihre

74 Bei der deutschen Übertragung des Terminus folge ich Willems \& Coene (2003). 
gemeinsame Konstitution eines Konstrukt-Frames. Ist Koerzion zu verzeichnen, muss also die Existenz und Auswirkung eines Konstruktions-Frames angenommen werden:

[F]rom the point of view of Construction Grammar, coercion constitutes a major argument in favor of the existence of constructions as independent form/meaning pairings, since it can be used as a heuristic means to discover the independent constructional semantics. If a construction is able to change the meaning of a lexical item that occurs in it, then one is entitled to say that the construction has a particular meaning on its own, irrespective of the lexical items that instantiate the construction. (Lauwers \& Willems 2011: 1220)

Koerzionseffekte sind demnach „prime examples of holistic meaning.“ (Audring \& Booij 2016: 623). Sie sind ein gutes Beispiel dafür, „wie eine syntaktische Konstruktion die konventionelle Bedeutung eines Lexems [...] unterdrückt und eine Reinterpretation erzwingt.“ (Albert 2015: 538). Wie Boas (2011a: 1284-1285) argumentiert, muss dies aber nicht nur einzelne Wörter (insbesondere Verben) betreffen, sondern kann sich über das gesamte Konstrukt einer ArgumentstrukturKonstruktion erstrecken und somit auch andere LE, die als Instanzen von KE, KEE und KorE in einem Konstrukt auftreten, betreffen. Von solchen einzelnen Koerzionseffekten ist das Koerzionspotenzial einer Konstruktion als Ganzes zu unterscheiden, nach dem dieser Parameter benannt ist.

Diese Unterscheidung möchte ich in Unterabschnitt 5.5.1 begründen, bevor ich in Unterabschnitt 5.5.2 auf die graduelle Natur von Koerzion eingehe - eine Erkenntnis, die eine wesentliche Voraussetzung für die empirische Messung des Koerzionspotenzials (Abschnitt 7.4) darstellt. In diesem Abschnitt stehen somit grundlegende Fragen zu diesem Parameter im Fokus. Auf eine Methode zur Messung des Koerzionspotenzials gehe ich in Unterabschnitt 7.4.1 und 7.4.3 ein, die empirischen Ergebnisse des Koerzionspotenzials für die drei untersuchten Konstruktionen sind Gegenstand der Unterabschnitte 7.4.2 bis 7.4.4.

\subsubsection{Koerzionseffekte und Koerzionspotenzial}

Um Koerzion festzustellen, ist es notwendig, einen Konstrukt-Frame daraufhin zu überprüfen, ob der in ihm enthaltene lexikalische Frames von dem KonstruktionsFrame angepasst ist (Unterabschnitt 4.4.1) und ob somit ein von dem reinen lexikalischen Frame abweichender Konstrukt-Frame vorliegt. Voraussetzung hierfür ist die Betrachtung der Parallelen zwischen den Strukturelementen der Konstrukte einer Konstruktion und den Frames, die an entsprechenden Konstrukt-Frames beteiligt sind. Die Motivierungen dieser Strukturelemente durch FE werden durch eine Betrachtung der lexikalischen Frames und des Konstruktions-Frames sowie 
deren Verhältnissen über einzelne Konstrukt-Frames hinweg ermittelt. Auf diesen Aspekt und eine Möglichkeit zu seiner methodischen Operationalisierung gehe ich in Kapitel 6 ausführlich ein, möchte zunächst aber den Begriff des Koerzionspotenzials erörtern.

Gemäß des Override Principle von Michaelis (2004: 25) könnte man eine potenzielle Dominanz des Konstruktions-Frames über einen lexikalischen Frame, den eine LE evoziert, annehmen: „[T] he argument is that larger linguistic structures are a stronger force than the individual word and are therefore able to modify properties of the coerced item.“ (Audring \& Booij 2016: 628). Die möglichen Abweichungen können dabei unterschiedlich stark sein, denn Koerzion ist keine binäre Eigenschaft, sondern ein graduelles Phänomen und ,the overwelming majority of coercions is so subtle that they quite go unnoticed.“ (Audring \& Booij 2016: 620). Eine Analyse kann also nicht damit enden, festzustellen, dass in einem Konstrukt Koerzion vorliegt oder nicht, vielmehr muss die konkrete Höhe eines solchen Koerzionseffekts messbar sein. Dies ist der erste Grund dafür, diesen Parameter Koerzionspotenzial zu nennen, denn auf der Ebene der Konstruktion hängt die Höhe dieses Potenzials davon ab, wie die Verteilung unterschiedlich hoher Koerzionseffekte über die Konstrukte der Konstruktion hinweg beschaffen ist. ${ }^{75}$

Der zweite Grund, von einem Koerzionspotenzial zu sprechen, setzt gerade bei der Beobachtung an, dass sich die Koerzionseffekte über die Konstrukte einer Konstruktion hinweg in unterschiedlicher Höhe verteilen. Aufgrunddessen halte ich es für sinnvoll, den Begriff des Koerzionspotenzials von einzelnen Koerzionseffekten abzugrenzen. Wie Bybee (2010) feststellt, wird Koerzion üblicherweise ausschließlich auf Ebene einzelner Konstrukte diagnostiziert, also auf Token-Ebene, nicht jedoch auf Ebene der Konstruktion und damit auf Type-Ebene:

The coercion theory seems to assume that instances of use that demand coercion have no effect on the meaning of a category. A usage-based approach, however, would propose that instances of language use have an effect on the more permanent representation of meaning. (Bybee 2010: 186)

Terminologisch möchte ich aus diesem Befund die Konsequenz ziehen, einzelne Fälle von Koerzion als Koerzionseffekte auf Token-Ebene für ein Konstrukt und das Koerzionspotenzial als eine Eigenschaft der Konstruktion, also auf TypeEbene, zu verstehen. Somit lässt sich dem Umstand Rechnung tragen, dass „coercion does not have to happen anew with each instance of use“ (Bybee 2010:

75 Bei Michaelis (2003a: 305) erscheint ebenfalls der Terminus „coercive potential“, bei Pustejovsky \& Jezek (2008: 207) und Michaelis (2011: 1393) ist die Rede von „coercion potential“. In allen Fällen ist jedoch keine genaue Definition oder eine Begründung für die Verwendung des Begriffs des Potenzials zu finden. 
186), sondern dass man die Eigenschaft, Konstrukte mit Koerzionseffekten hervorzubringen, einer Konstruktion zuschreiben kann und dies in die semantische Beschreibung einer Konstruktion eingehen lassen sollte. Vorschläge dafür, wie sich von einzelnen Koerzionseffekten auf das Koerzionspotenzial einer Konstruktion schließen lässt, Koerzionseffekte sich also generalisieren lassen, unterbreite ich in den Unterabschnitten 7.4.1 und 7.4.3.

Begrifflich fassen lassen sich Koerzionseffekte mit der von Audring \& Booij (2016: 628-634) vorgeschlagenen dreiteiligen Typologie von coercion by selection, coercion by enrichment und coercion by override: ${ }^{76}$ Ersteres Phänomen bezeichnet einen recht schwachen Koerzionseffekt, in dem bestimmte Aspekte des Bedeutungspotenzials einer LE - also des lexikalischen Frames, den sie evoziert (vgl. Unterabschnitt 4.2.2) - selektiert werden, im zweiten Phänomen wird der lexikalische Frame einer LE durch den Konstruktions-Frame erweitert, während letzteres Phänomen den stärksten Koerzionseffekt einer (mehr oder weniger) vollständigen Überschreibung eines lexikalischen Frames durch den Konstruktions-Frame bezeichnet. Michaelis' Override Principle erfasst demnach nur eine der möglichen Erscheinungsformen von Koerzion (vgl. Audring \& Booij 2016: 627-628). Auch auf diese Einteilung komme ich in den Unterabschnitten 7.4.1 und 7.4.3 zurück.

\subsubsection{Koerzion als graduelles Phänomen}

Bevor die Höhe des Koerzionspotenzials der drei untersuchten Konstruktionen auf Grundlage der Untersuchung der Strukturparallelen zwischen ihren KtE und KEE auf der einen Seite sowie den FE ihres Konstruktions-Frames und der unterschiedlichen lexikalischen Frames auf der anderen Seite bestimmt werden kann (Abschnitt 7.4), ist es an dieser Stelle wichtig, die graduelle Natur von Koerzion, ${ }^{77}$ wie sie sich exemplarisch in den Konstrukten der reflexiven Bewegungskonstruktion äußert, festzuhalten. Schon die way-Konstruktion gilt als ein Paradefall für Koerzion (vgl. Michaelis 2003a: 276, 2005: 59; Traugott \& Trousdale 2013: 86). Geht man davon aus, dass die Konstruktion formal in erster Linie durch eine syntagmatische Abfolge der KE BEWEGENDES, EREIGNIS, WEG und des KEE gekennzeichnet ist, so ist der entstehende Koerzionseffekt, den sie erzeugen kann, stark abhängig

76 Vgl. auch die Unterscheidung zwischen inherent compatibility und semantic enrichment bei Perek (2015: 28) sowie die Typologie von Engelberg (2019: 20-21), der neologistische, konventionalisierte und musterbildende Koerzionseffekte unterscheidet. Eine Methode zu deren präziser Abgrenzung fehlt allerdings.

77 Zur graduellen Natur von Koerzion vgl. überblickend auch Lauwers \& Willems (2011: 12271230). 
von den KtE, die das KE EREIGNIS instanziieren können - mit anderen Worten: von den Präferenzen für lexikalische Frames (Unterabschnitt 5.3.3). Gleichzeitig ist die formale Abstraktheit der Konstruktion (Abschnitt 5.1) entscheidend, da nur ein KE wie EREIGNIS in der Lage ist, durch KtE instanziiert zu werden, die als LE entsprechende lexikalische Frames evozieren. Diese Beobachtungen verknüpfen den semantischen Parameter des Koerzionspotenzials mit den Parametern der formalen Abstraktheit und der Beschränkungen und Präferenzen, wie es in Abbildung 5.1 im Eingang zu diesem Kapitel dargestellt ist.

Treten in das KE EREIGNIS nun LE ein, deren lexikalischer Frame dem Konstruktions-Frame, also Motion, entspricht, so entsteht keine Koerzion. Beispiele hierfür sind die Belege in (84) mit den LE bewegen (move.v) und begeben (go.v), die das KE EREIGNIS instanziieren.

(84) a. Er [Motion bewegte] sich durch vermeintlich unauffällige harmonische Räume mit einer Sehnsucht nach Farbigkeit, die alle Grauwerte des Theoretischen löschte. (Die Zeit, 10.02.2000, Nr. 7)

b. Titus legte die drei Blätter übereinander, faltete sie sorgfältig zusammen und [Motion begab] sich, wie von Petersen gefordert, zurück auf seinen Platz. (Schulze, Ingo: Neue Leben, Berlin: Berlin Verlag 2005, S. 782)

c. Oder aber Sie [Motion begeben] sich unter die Langschläfer, denn in den Halbschlafphasen am späteren Morgen wird auch viel geträumt. (Die Zeit, 20.04.2000, Nr. 17)

Bei Motion als lexikalischem Frame handelt es sich, da er identisch mit dem Konstruktions-Frame ist, um einen relatierten lexikalischen Frame (vgl. Unterabschnitt 5.4.2). Für ihn wie für alle relatierten lexikalischen Frames, auch diejenigen mit Frame-Nähen von +1 und mehr, gilt, dass sie keine Koerzionseffekte erzeugen. Werden in den Konstrukten allerdings LE gebraucht, die unrelatierte lexikalische Frames evozieren, so entstehen Koerzionseffekte. Bei dieser binären Unterscheidung kann es allerdings, wie in Unterabschnitt 5.5.1 angedeutet, nicht bleiben, denn die graduelle Natur des Phänomens der Koerzion äußert sich in unterschiedlichen Höhen von Koerzionseffekten in Konstrukten mit unrelatierten lexikalischen Frames. Diese Koerzionseffekte bestehen vor allem in coercion by enrichment und coercion by override im Sinne von Audring \& Booij (2016: 628-634). Eine einfache Form der ,Erweiterung' eines lexikalischen Frames durch einen Konstruktions-Frame liegt vor, wenn bestimmte Strukturelemente des Konstrukts durch FE beider Frames zugleich motiviert werden, also eine doppelte Motivierung (Unterabschnitte 6.2.3 und 6.3.3) vorliegt. Dies ist etwa bei dem lexikalischen Frame Manipulate_into_doing der Fall: Das Konstrukt in (85) zeigt, dass das KtE 
des KE WEG durch FE des lexikalischen Frames und des Konstruktions-Frames doppelt motiviert wird.

(85) Der Fabrikant breitete sein Schweigen wieder aus, der Offizier [Manipulate_ into_doing mogelte] sich [Resulting_Action aus seinem Sitz Source]. (Düffel, John von: Vom Wasser, München: dtv 2006, S. 56)

Die doppelte Motivierung ist allerdings nicht nur für KtE des KE WEG möglich, sondern ebenso für das KEE. Wird dies gleichzeitig zum KtE des KE WEG ebenfalls doppelt motiviert, ist der Koerzionseffekt höher. Dieser Fall ist etwa in Konstrukten mit den lexikalischen Frames Cause_to_amalgamate, Attaching oder Smuggling zu beobachten, die die Konstrukte in (86) illustrieren sollen.

a. [PART_2 In das Weiß unter Blau ${ }^{\text {GoAL}}$ ] [Cause_to_amalgamate mischen] [PART_1

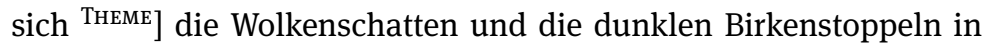
den Senken. (Die Zeit, 05.01.2000, Nr. 2)

b. Spinnenweb [Attaching klebte] [Iтем sich ${ }^{\text {Theme] [ }}{ }_{\text {Goal }}$ in sein Gesicht GoAL]. (Koneffke, Jan: Paul Schatz im Uhrenkasten, Köln: DuMont Buchverlag 2000, S. 216)

c. Und wenn die Jungs nach getaner Tat in den Armen der Liebsten schlummern, [smuggling schmuggelt] [Goods sich THEmE] Rudi mittels Dollars und Autogrammfußbällen am Endlosstau von Brest vorbei [GoAL nach Polen ${ }^{\text {GoAL }}$ ] rein. (Die Zeit, 24.02.2000, Nr. 9)

Gegenüber diesen Fällen von coercion by enrichment, in denen Anteile des lexikalischen Frames bis zu einem gewissen Grad noch immer im Konstrukt-Frame ,übrig bleiben', stellen Fälle von coercion by override die höchsten Koerzionseffekte dar. ${ }^{78}$ Sie liegen vor, wenn in den beiden soeben bereits betrachteten Strukturelementen, dem KtE des KE WEG sowie dem KEE, keine Motivierung des lexikalischen Frames mehr zu finden ist, sondern diese ausschließlich durch den KonstruktionsFrame geleistet wird (im Sinne der in Unterabschnitt 4.4.1 diskutierten Variante c einer Frame-Anpassung). Ein solcher Fall ist in den Konstrukten in (87) mit den lexikalischen Frames Daring, Hostile_encounter und Work zu sehen. Die Höhe des

78 Diese beiden Fälle sind somit eine Feindifferenzierung dessen, was Perek (2015: 28) allgemein unter semantic enrichment fasst: „In the case of semantic enrichment, the respective contributions of the verb and the construction are distinct: the verb conveys what it 'normally' does and the missing elements of meaning are provided by the construction." Frame-semantisch gewendet handelt es sich dabei um „cases where the use of a verb involves facets of meaning that are arguably absent from its semantic frame [...], and are contributed by the construction." (Perek 2015: 28). 
Koerzionseffekts ist also nicht allein abhängig von der semantischen Motivierung eines KtE wie desjenigen des KE WEG: Die semantische Motivierung der Instanz des KEE muss ebenfalls mit in Betracht gezogen werden.

a. Aber früher, als wir noch klein waren, [Daring traute] [sich ${ }^{\text {THEME }}$ niemand [an uns ${ }^{\mathrm{GOAL}}$ ] heran, auch nicht in Mückes Gegend, denn wir standen unter dem Schutz seines Bruders, und dessen Rache würde fürchterlich sein. (Goosen, Frank: Liegen lernen, Frankfurt am Main: Eichborn AG 2000, S. 88)

b. Der Wagen legte kaum zweihundert Meter in der Minute zurück,

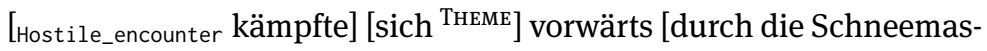
sen ${ }^{\text {PATH }}$, bog von der Landstraße ab in eine von wenigen Laternen beleuchtete Allee. (Krausser, Helmut: Eros, Köln: DuMont 2006, S. 8)

c. „Manchmal [work arbeiten] [sich ${ }^{\text {THEME}] ~[a u s ~ d e r ~ t e i g i g e n, ~ f o r m l o s e n ~}$ Volksmasse SouRCE] irgendwelche besonderen, starken, sehr arbeitsfähigen Menschen nach oben. (Schlögel, Karl: Petersburg, München Wien: Carl Hanser Verlag 2002, S. 292)

Diese wenigen Beispiele verdeutlichen, dass Koerzionseffekte über die Konstrukte einer Konstruktion hinweg in unterschiedlicher Höhe verteilt sein können, je nachdem, wie die einzelnen Konstrukt-Frames konstituiert sind. Einer Konstruktion kommt deshalb nicht eine über alle Konstrukte stabile, fest zuzuschreibende Eigenschaft von Koerzion zu, sondern sie wird durch ein Koerzionspotenzial gekennzeichnet, das je nach Verteilung der Koerzionseffekte über die Konstrukte hinweg spezifisch ausfällt. Dieses Koerzionspotenzial lässt eine Aussage über die Wirkung des Konstruktions-Frames auf die Konstitution einzelner KonstruktFrames zu, in denen der Konstruktions-Frame im Sinne von Michaelis (2005: 50) ,durchscheint'.

Ausgangspunkt für die Messung des Koerzionspotenzials ist eine einfache Methode: Um einen Koerzionseffekt festzustellen und seine Höhe zu bestimmen, muss ein gegebenes Konstrukt daraufhin untersucht werden, wie groß der Anteil des lexikalischen Frames ist, der nach einer Frame-Anpassung noch innerhalb eines Konstrukt-Frames ,übrig bleibt‘. Die verschieden hoch ausgeprägten Koerzionseffekte definieren sich also nach der Konstitution des Konstrukt-Frames. Das gesamte Koerzionspotenzial lässt sich durch quantitative Aussagen über die Verteilung der Koerzionseffekte in den Konstrukten ermitteln. Die Konstrukte einer Konstruktion müssen, wie an den Belegen in (85)-(87) demonstriert, daraufhin untersucht werden, wie hoch der Koerzionseffekt jeweils ist. Es wird deutlich, dass sich diese Untersuchung wesentlich auf Erkenntnisse über die Strukturparallelen zwischen KtE und KEE der Konstrukte und den FE der in den Konstrukt- 
Frames involvierten Frames stützen muss: Je nachdem, wie die semantische Motivation der KtE und KEE in einem Konstrukt zusammengesetzt ist - durch den lexikalischen Frame allein, durch den Konstruktions-Frame allein, oder durch beide zugleich -, ist der Koerzionseffekt höher oder niedriger. Die in den Konstrukten in (85)-(87) illustrierten Koerzionseffekte zeigen nur drei Kombinationen solcher semantischer Motivierungen, es lassen sich für die drei untersuchten Konstruktionen jedoch insgesamt sieben solcher Kombinationen feststellen, auf die ich in den Unterabschnitten 7.4.1 und 7.4.3 als sieben Koerzionsstufen ausführlicher eingehe.

Eine Untersuchung der semantischen Motivierungen der Konstrukte einer Konstruktion, also der Konstitution ihrer Konstrukt-Frames, ist eine konstruktionssemantisch leicht umsetzbare Methode, um Koerzionseffekte festzustellen und ihre Höhe zu bestimmen. Die Analyse lexikalischer Frames und des Konstruktions-Frames lässt sich allerdings noch durch Aussagen über die aus ihnen resultierenden lexikalischen Bedeutungen und die Konstruktionsbedeutung erweitern, denn Koerzion ist ein Phänomen, das ebenso die aus diesen Frames hervorgehenden Bedeutungen betrifft. Deshalb weist Ziem (2018b: 80-81) darauf hin, dass Koerzionseffekte grundsätzlich mit der auf Minsky (1975: 212, 1988: 247) zurückgehenden frame-semantischen Idee der Standardwerte in Verbindung zu bringen sind, die einen lexikalischen Frame oder einen Konstruktions-Frame zu einer lexikalischen Bedeutung oder einer Konstruktionsbedeutung hin spezifizieren (vgl. Unterabschnitt 4.1.1). Die ,Verdrängung' oder Modifikation von Bestandteilen einer lexikalischen Bedeutung - oder: ihrer Grundlage in Gestalt von FE eines lexikalischen Frames - durch die Konstruktionsbedeutung kann gleichzeitig als ein Überschreiben möglicher Standardwerte der lexikalischen Bedeutung verstanden werden.

Coercion, [...], macht nicht nur versteckte Standardwerte sichtbar; ebenso wichtig ist, dass Coercion auch Hinweise auf Mechanismen der Tilgbarkeit von Standardwerten liefert. (Ziem 2018b: 81)

Voraussetzung dafür, dass ein Standardwert getilgt werden kann, ist, dass er von einem anderen Standardwert überschrieben wird. Dies geschieht dann, wenn ein KtE oder KEE eines Konstrukts doppelt motiviert wird, also von einem FE des lexikalischen Frames und von einem FE des Konstruktions-Frames (Unterabschnitte 6.2.3 und 6.3.3). Der Standardwert des FE des lexikalischen Frames wird durch den Standardwert des FE des Konstruktions-Frames getilgt. Anhand der LE fressen (etwa: eat.v), die ich in Abschnitt 4.2 zur Illustration von Standardwerten herangezogen habe, möchte ich dies an der reflexiven Bewegungskonstruktion verdeutlichen. In (88) wird das KtE des KE WEG durch zwei FE zugleich moti- 
viert: Ingestion.IngESTIBLES des lexikalischen Frames und Motion.PATH des Konstruktions-Frames.

(88) Die nämlich erreichte im 18. Jahrhundert mit einem aus Amerika kommenden Schiff die französische Küste und [Ingestion fraß] sich unbarmherzig [Ingestibles durch die französischen Weinäcker ${ }^{\mathrm{PATH}}$ ]. (Die Zeit, 30.03.2000, Nr. 14)

Der Standardwert des FE Ingestion.INGESTIBLEs könnte bei der in (88) vorliegenden lexikalischen Bedeutung von fressen in Bezug auf das Referenzobjekt, auf das das FE Ingestion.INGESTOR verweist - eine Reblaus - etwa ,Weinblätter ${ }^{6}$ lauten. ${ }^{79}$ Mehrere Weinäcker gehören - auch für einen ganzen Schwarm von Rebläusen sicher nicht dazu, ganz zu schweigen von allen oder auch nur den meisten französischen Weinäckern. ${ }^{80}$ Dadurch, dass nun aber das KtE durch die französischen Weinäcker zugleich durch das FE Motion.PATH motiviert wird, wird es im Sinne des KE WEG reinterpretiert. Was die Rebläuse fressen, sind freilich nicht ganze Weinäcker, sondern lediglich Teile davon, wobei sie sich (vermutlich weniger metaphorisch als es auf den ersten Blick scheint) von einem Weinacker aus zu anderen Weinäckern ,bewegen'. Der Standardwert für das FE Ingestion.INGESTIBLES wird also durch das FE Motion.GoAL getilgt zugunsten einer Konstruktbedeutung im Sinne der reflexiven Bewegungskonstruktion.

Diese kurze Analyse zeigt, dass Standardwerte, wie Busse (2012: 263) feststellt, ,jederzeit kontextuell (z.B. durch nachträglich einlaufende Informationen) korrigiert bzw. überformt werden können.“ Eine solche (zusätzliche) Information ist in diesem Fall die Konstruktionsbedeutung, die zu der lexikalischen Bedeu-

79 Da zu der lexikalischen Bedeutung von fressen freilich noch andere Standardwerte des FE Ingestion.INGESTIBLES gehören, je nachdem, auf welches ,fressende Lebewesen` Bezug genommen wird, könnte man präziser auch von einem Wertebereich von Standardwerten im Sinne von Busse (2012: 565-572) sprechen.

80 Mit anderen Worten: Sie liegen außerhalb des Wertebereichs der Standardwerte für das FE Ingestion.INGESTIBLES mit der lexikalischen Bedeutung von fressen. 
tung hinzutritt. Der dadurch ausgelöste Koerzionseffekt resultiert in der lokalen Anpassung ${ }^{81}$ des Standardwerts. ${ }^{82}$

Der Einbezug von Standardwerten ist wichtig, um die Messung des Koerzionspotenzials nicht allein auf der Basis von FE und somit auf den Ebenen von lexikalischem Frame und Konstruktions-Frame zu belassen, sondern sie weiter $\mathrm{zu}$ differenzieren. Um dies allerdings systematisch und vor allem quantitativ durchführen zu können, sind umfangreiche Analysen lexikalischer Bedeutungen und damit von Standardwerten lexikalischer Frames nötig, für die gegenwärtig keine Datengrundlage besteht (vgl. auch Dux 2020: 70). Bei der Messung des Koerzionspotenzials einer Konstruktion sehe ich deshalb von einem Einbezug von Standardwerten ab und belasse die Argumentation auf Ebene der Frames. In Abschnitt 7.4, der sich mit der Messung des Koerzionspotenzials für alle drei untersuchten Konstruktionen und dem Eingang dieses Parameters in einen Konstruktionseintrag beschäftigt, stehen deshalb Konstrukt-Frames und ihre Konstitution aus lexikalischen Frames und Konstruktions-Frames im Vordergrund.

\subsection{Produktivität}

Die Produktivität einer Konstruktion ist für einen semantischen Parameter von Konstruktionen relevant, weil sie Fragen nach der Instanziierbarkeit einer Konstruktion aus semantischer Sicht beantwortet. Sie schließt damit zunächst direkt an den Parameter der Präferenzen für lexikalische Frames (Abschnitt 5.3) an, denn die Produktivität einer Konstruktion bemisst sich wesentlich danach, welche lexikalischen Frames in die Konstitution eines Konstrukt-Frames involviert sein können. ${ }^{83}$ Gleichzeitig sind diese lexikalischen Frames vor dem Hintergrund

81 Koerzionseffekte sind demnach auch in dieser Hinsicht wesentliche Auswirkungen von Frame-Anpassungen, die ich in Unterabschnitt 4.4.1 im Anschluss an den Begriff des Anpassungsprozesses (matching process) im Sinne von Minsky (1975: 213) und seine Weiterentwicklung durch Busse (2012: 624-627) diskutiert habe. Nicht zufällig verwendet etwa Goldberg (1995: 159) den Terminus accommodation synonym zu demjenigen der Koerzion. Die konstruktionssemantische Konsequenz daraus ist, dass sich die sieben Koerzionsstufen, die ich in den Unterabschnitten 7.4.1 und 7.4.3 vorstelle, nach der Höhe der Frame-Anpassung eines lexikalischen Frames durch den Konstruktions-Frame zusammensetzen.

82 Zur Tilgung von Standardwerten ist zu sagen, dass sie, wie soeben gezeigt, nur bei angepassten Konstrukt-Frames (Unterabschnitt 4.4.1) funktioniert, nämlich solchen, die sowohl aus FE des (unrelatierten) lexikalischen Frames als auch solchen des Konstruktions-Frames bestehen und bei denen ein KtE und/oder KEE durch zwei FE dieser beiden Frames doppelt motiviert wird. 83 Implizit spiegelt sich hierin auch der Zusammenhang zwischen der formalen Abstraktheit der Konstruktion und ihrer Produktivität wider, für den etwa Clausner \& Croft (1997: 255) argumen- 
des Koerzionspotenzials einer Konstruktion (Abschnitt 5.5) an den KonstruktionsFrame rückgekoppelt: Je produktiver eine Konstruktion ist, desto potenziell mehr Konstrukte zeigen eine Frame-Anpassung des lexikalischen Frames durch den Konstruktions-Frame hin zu einem Konstrukt-Frame. Koerzionspotenzial und Produktivität hängen somit direkt zusammen, denn ist das Koerzionspotenzial einer Konstruktion hoch, spricht dies gleichzeitig für eine hohe Produktivität (vgl. auch Suttle \& Goldberg 2011: 1238) und ebenso für einen großen Einfluss des Konstruktions-Frames gegenüber den lexikalischen Frames auf die Konstitution von Konstrukt-Frames (vgl. die Ergebnisse in den Unterabschnitten 7.4.2 bis 7.4.4 sowie 7.5.2). Aus diesem Grund muss die Produktivität einer Konstruktion Bestandteil einer konstruktikographischen Beschreibung sein.

Als Grundlage für die konstruktionssemantische Betrachtung von Produktivität als Gegenstand eines semantischen Parameters von Konstruktionen soll der Produktivitätsbegriff von Barðdal (2008) dienen. Dieser besteht aus zwei Komponenten: (i) Type-Frequenz und (ii) semantischer Kohärenz. Im Folgenden möchte ich insbesondere den Aspekt der semantischen Kohärenz in den Blick nehmen, um den Produktivitätsbegriff im Rahmen einer Konstruktionssemantik genuin semantisch zu bestimmen. Dazu ziehe ich die Einteilung lexikalischer Frames nach ihrer Frame-Nähe zum Konstruktions-Frame in relatierte und unrelatierte Frames (Unterabschnitt 5.4.2) heran. In Unterabschnitt 5.6.1 argumentiere ich zunächst, dass die Prototypikalität lexikalischer Frames deren semantische Kohärenz abbildet. Im Anschluss daran diskutiere ich in Unterabschnitt 5.6.2 die Gründe dafür, warum unrelatierte lexikalische Frames die Basis für die Erweiterbarkeit einer Konstruktion sind, welche Barðdal (2008: 1) als definitorisch für Produktivität ansieht. Die auf das Kriterium der Type-Frequenz bezogene Messung der Produktivität, die für eine konstruktikographische Beschreibung benötigt wird, sowie den konkreten Vergleich der untersuchten Konstruktionen thematisiere ich in den Unterabschnitten 7.5.1 und 7.5.2.

\footnotetext{
tieren, allerdings bezogen auf die lexikalische Spezifiziertheit (Unterabschnitt 5.1.2) als Kriterium für ihre formale Abstraktheit. Je weniger lexikalisch spezifiziert eine Konstruktion ist, desto höher muss demnach ihre Produktivität sein (vgl. auch Bybee 1995: 430, 432; Ziem \& Lasch 2013: 105). Da bereits die Präferenzen für lexikalische Frames eine Aussage über die lexikalische Spezifiziertheit der Konstruktion treffen (je geringer die Spezifiziertheit, desto potenziell breiter die Präferenzen für lexikalische Frames) nehme ich den Zusammenhang zwischen Abstraktheit und Produktivität nicht gesondert in den Blick.
} 


\subsubsection{Prototypikalität: relatierte lexikalische Frames}

Mit der Produktivität einer Konstruktion ist konstruktionssemantisch gesehen der mögliche Eintritt eines lexikalischen Frames in die Konstitution eines KonstruktFrames gemeint. Um diesen Eintritt zu beobachten, muss für die drei untersuchten Konstruktionen das Augenmerk auf ein bestimmtes KE gelegt werden, das für alle drei Konstruktionen identisch ist: das KE EREIGNIS. Da dieses KE durch LE instanziiert wird, die den lexikalischen Frame evozieren (dazu auch Unterabschnitt 7.3.1), ist die Produktivität einer Konstruktion an ebendiesen lexikalischen Frames - genauer: an den Präferenzen für diese lexikalischen Frames (Unterabschnitt 5.3.3) - zu messen. Schon Ziem (2018a) betont, dass sich die Produktivität nach lexikalischen Präferenzen eines bestimmten KE, im Falle der drei untersuchten Konstruktionen also EREIGNIS, richtet:

Die Produktivität einer Konstruktion betrifft die Variation der Leerstellenfüllung, also die Menge an unterschiedlichen Fillern (Instanzen), die in einer Konstruktion realisiert werden können. Die Produktivität einer Konstruktion erhöht sich in dem Maße, wie die Anzahl an unterschiedlichen Instanzen steigt, die die Leerstellen füllen. [...] Zwischen Constraints der Leerstellen-Füllung und der Produktivität einer Konstruktion besteht mithin ein direkter Zusammenhang. (Ziem 2018a: 10)

Grundlage für den Produktivitätsbegriff, wie Ziem ihn verwendet und wie auch ich ihn verwende, bildet die Konzeption von Barðdal (2008), die (syntaktische) Produktivität definiert als ,an argument structure construction's ability to attract new or existing lexical items, i.e. a construction's ExTENSIBILITy.“ (Barðdal 2008: 1). Anders als Barðdal füge ich an dieser Stelle allerdings bereits eine Generalisierung ein: Die Produktivität einer Konstruktion bemisst sich sinnvollerweise in erster Linie nicht nach der Erweiterbarkeit um einzelne $L E$ und deren lexikalische Bedeutungen, sondern um lexikalische Frames, also Gruppen von LE, die gemeinsam ebendiesen Frame evozieren und potenziell über unterschiedliche lexikalische Bedeutungen verfügen können (vgl. Unterabschnitt 4.1.1). ${ }^{84}$ Erweiterbarkeit ist in diesem Zusammenhang also nichts anderes als der Grad, inwieweit eine Konstruktion die Aufnahme, neuer' lexikalischer Frames in ihre Konstrukt-Frames ermöglicht. Ausschlaggebend für die Messung der Produktivität einer Konstruktion sind also die lexikalischen Frames, die in die Konstitution von Konstrukt-Frames eingehen.

84 Auch andere Produktivitätsbegriffe operieren lediglich auf der Ebene von LE, ohne sie hinsichtlich ihrer lexikalischen Frames zu generalisieren. So fasst Engelberg (2019: 18) zusammen, „dass sich Produktivität darin ausdrückt, dass mit zunehmender Korpusgröße auch weitere neue, bisher dort nicht beobachtete lexikalische Einheiten in Slots auftreten“. 
Gemäß dem Ansatz von Barðdal (2008) wird Produktivität wesentlich durch zwei Faktoren bestimmt: Type-Frequenz ${ }^{85}$ und semantische Kohärenz. ${ }^{86}$

The hypothesis that I will be pursuing in this study is that the type frequency of a schema and its coherence appropriately predict a schema's productivity (in the sense of 'extensibility'). In other words, productivity is a function of both type frequency and coherence. (Barðdal 2008: 27)

Relatierte lexikalische Frames bilden nun das ab, was hier mit Kohärenz gemeint ist: Sie bilden eine Gruppe semantisch kohärenter lexikalischer Frames, die in Konstrukt-Frames eingehen können. Dies entspricht ziemlich genau der von Barðdal (2008: 27) verwendeten Definition von Kohärenz: „By schema coherence I mean the internal consistency found between all the members of each schema or a category." Mit anderen Worten: Die über Frame-Nähen determinierte Prototypikalität lexikalischer jener Frames (Unterabschnitt 5.4.2) ist ein Maßstab für die semantische Kohärenz einer Konstruktion. Darin besteht ein Grund zur Verknüpfung des Parameters der Produktivität mit demjenigen der Frame-Nähe, wie es in Abbildung $5.1 \mathrm{im}$ Eingang zu diesem Kapitel dargestellt ist. Semantische Kohärenz äußert sich zudem auch in der hier bereits enthaltenen Generalisierung (vgl. Abbildung 5.3 in Unterabschnitt 5.3.3): Auch die LE, die einem lexikalischen Frame zugeordnet werden, können auf einer ersten Ebene als semantisch kohärent gelten, wenngleich sie unterschiedliche lexikalische Bedeutungen aufweisen. Erst auf einer zweiten Ebene würde deren Feindifferenzierung nach jenen lexikalischen Bedeutungen innerhalb eines lexikalischen Frames erfolgen. Übertragen auf alle LE der relatierten Frames lässt sich somit festhalten, dass alle diese LE und damit alle Konstrukte, die einen relatierten lexikalischen Frame evozieren, als semantisch kohärent zu verstehen sind. Daraus folgt ebenso, dass alle relatierten lexikalischen Frames noch nicht zur Erweiterbarkeit einer Konstruktion zählen, jedoch können auch sie bereits Aufschluss über die Produktivität der Konstruktion geben.

Belege für semantisch kohärente lexikalische Frames habe ich für die reflexive Bewegungskonstruktion bereits in Unterabschnitt 5.4.3 zitiert, eine systematische Analyse wird in Kapitel 6 hinsichtlich der Strukturparallelen zwischen der

85 Für das Kriterium der Type-Frequenz vgl. auch Bybee (1985: 133, 1995: 430, 2010: 67), Clausner \& Croft (1997: 254), Suttle \& Goldberg (2011: 1242), Hilpert (2018: 95) und Diessel (2019: 130-131). 86 Bei Diessel (2019: 126-130) entspricht das Kriterium der semantischen Kohärenz in etwa dem Begriff der semantischen Ähnlichkeit, wobei diese nicht nur - wie in meinem Ansatz - das Verhältnis zwischen (in Termini meines Modells) lexikalischen Frames und dem Konstruktions-Frame betreffen muss, sondern auch das Verhältnis lexikalischer Frames untereinander. Als ein Beispiel zieht Diessel explizit die way-Konstruktion heran. 
Konstruktion und den Frames, die ihre Konstrukte semantisch motivieren, folgen. $\mathrm{Zu}$ Illustrationszwecken interessant ist nun jedoch zu sehen, wie weit die semantische Kohärenz einer Konstruktion reichen kann. Zu beobachten ist dies an lexikalischen Frames, die zwar noch zum Konstruktions-Frame relatiert sind, allerdings innerhalb der Klasse dieser relatierten lexikalischen Frames die niedrigste FrameNähe und damit geringste Prototypikalität aufweisen.

Für die reflexive Bewegungskonstruktion sind Frame-Nähen von bis $\mathrm{zu}+3$ belegt. Genauer gesagt handelt es sich dabei um ein einziges Konstrukt mit dem lexikalischen Frame Making_faces, der zum Konstruktions-Frame Motion in BenutztRelation steht und in dieser Relation eine Frame-Nähe von +3 aufweist. Der entsprechende Beleg findet sich in (89). Zwar ist die Frame-Nähe zu Motion mit +3 niedrig, das Konstrukt zählt aber aufgrund des relatierten Frames nach wie vor zur Menge der semantisch kohärenten, wenngleich weniger prototypischen, Konstrukte.

Ein polnisches Model (Goshia mit Namen) am Tisch gegenüber versucht sich seit 20 Minuten in unser Gespräch reinzu[Making_faces grinsen]. (Die Zeit, 30.03.2000, Nr. 14)

Das Konstrukt in (89) kann dadurch, dass das KE EREIGNIS durch ein Partikelverb (reingrinsen) instanziiert wird, zugleich als Konstrukt der reflexiven Partikelverbkonstruktion gelten. ${ }^{87}$ Für die reflexive Partikelverbkonstruktion ist darüber hinaus kein Konstrukt in vergleichbarer Frame-Nähe belegt, das Maximum liegt hier bei einer Frame-Nähe von +1 für die Vererbungsrelation, die Benutzt-Relation und die Siehe_auch-Relation (vgl. Tabelle 5.18 in Unterabschnitt 5.4.3). Für die reflexive Weg-Konstruktion beschränkt sich die Menge relatierter und damit semantisch kohärenter lexikalischer Frames gänzlich auf Motion, der durch die LE bahnen evoziert werden kann (vgl. schon Unterabschnitt 3.3.1).

Semantische Kohärenz ist in dem Ansatz von Barðdal (2008) allerdings nicht das einzige Kriterium für Produktivität. Hinzu kommt noch das Kriterium der Type-Frequenz:

By type frequency I mean the total number of types which can instantiate a construction. [...] For a syntactic construction, like for instance the ditransitive construction, all predicates which can instantiate the ditransitive construction together make up its type frequency. (Barðdal 2008: 27)

87 In den Korpusdaten für die reflexive Partikelverbkonstruktion ist es allerdings nicht enthalten, was daran liegt, dass ausschließlich nach finiten Partikelverben gesucht wurde und reinzugrinsen deshalb nicht erfasst wurde (vgl. Unterabschnitt 3.4.1). 
Damit ist deutlich, dass bereits eine hohe Type-Frequenz derjenigen lexikalischen Frames, die innerhalb der Konstruktion semantisch kohärent sind, also zu den relatierten lexikalischen Frames gehören, auf eine hohe Produktivität der Konstruktion hindeutet. Diese semantisch kohärenten lexikalischen Frames sind der einfachste Weg, eine Konstruktion produktiv zu verwenden, wenn man davon ausgeht, „dass die Produktivität einer Konstruktion umso stärker ist, je leichter es Sprechern fällt, neue Instanzen der Konstruktion zu produzieren oder zu prozessieren.“ (Hilpert 2018: 94). Der Produktions- und Verarbeitungsaufwand von semantisch kohärenten (also relatierten) lexikalischen Frames erscheint gegenüber unrelatierten und damit semantisch inkohärenten lexikalischen Frames geringer, weshalb sie bereits zur produktiven Erweiterbarkeit einer Konstruktion gehören müssen. Die Type-Frequenz relatierter und damit semantisch kohärenter lexikalischer Frames ist somit die eine Hälfte einer Methode zur Messung der Produktivität (Unterabschnitt 7.5.1). Die andere Hälfte betrifft lexikalische Frames, die außerhalb dieser semantischen Kohärenz liegen und unrelatiert sind. Unrelatierte lexikalische Frames zeigen jedoch, so möchte ich im folgenden Unterabschnitt 5.6.2 argumentieren, deutlicher als relatierte lexikalische Frames die Erweiterbarkeit einer Konstruktion und sind zur Messung der Produktivität deshalb noch ein wenig relevanter.

\subsubsection{Erweiterbarkeit: unrelatierte lexikalische Frames}

Obwohl bereits die relatierten und damit semantisch kohärenten lexikalischen Frames einen Hinweis auf die Produktivität der Konstruktion geben können, sagen sie noch nichts über den Einfluss des Konstruktions-Frames aus, denn aufgrund ihrer Prototypikalität (Unterabschnitt 5.4.2) und mehr noch den nicht vorhandenen Koerzionseffekten (Unterabschnitt 5.5.2) stellen sie keine Erweiterung der Konstruktion dar, wie sie im Produktivitätsbegriff von Barðdal (2008) gefordert ist. Eine deutliche Erweiterung liegt bei unrelatierten lexikalischen Frames vor. Mit anderen Worten: Wertvollere Aussagen über die Produktivität einer Konstruktion liefern unrelatierte lexikalische Frames. ${ }^{88}$ Damit beantwortet sich die be-

88 Vgl. dazu Suttle \& Goldberg (2011: 1238), die feststellen, dass der Begriff der Produktivität im engeren Sinne bisweilen lediglich für, übliche‘ Konstrukte (hier also: solche mit relatierten lexikalischen Frames) gebraucht wird, während für ,neue‘ Konstrukte (hier also: solche mit unrelatierten lexikalischen Frames) eher der Begriff der Koerzion verwendet wird. Da sich in Konstrukten mit unrelatierten lexikalischen Frames tatsächlich Koerzionseffekte äußern (vgl. Abschnitte 5.5 und 7.4), sehe ich unrelatierte lexikalische Frames gegenüber relatierten lexikalischen Frames als deutlicheren Hinweis auf die Produktivität einer Konstruktion an. 
reits implizit aufgeworfene Frage, was es heißt, dass eine Konstruktion um ,neue‘ Konstrukte erweitert wird. ,Neue‘ Konstrukte sind solche, bei denen ein unrelatierter lexikalischer Frame in die Konstitution des Konstrukt-Frames eingeht und im Zuge einer Frame-Anpassung angepasst wird. Dies ist ein weiterer Grund dafür, warum der semantische Parameter der Produktivität (wie in Abbildung 5.1 dargestellt) mit demjenigen der Frame-Nähe verknüpft ist. Die Generalisierung, die ich in Unterabschnitt 5.6.1 auf der Ebene semantisch kohärenter Konstrukte eingeführt habe, setzt sich hier nahtlos fort. Als ,neu' gelten nicht in erster Linie LE und deren lexikalische Bedeutungen, sondern lexikalische Frames. Ein lexikalischer Frame kann als ,neu' gelten, wenn er von den relatierten lexikalischen Frames abweicht, also unrelatiert ist. ${ }^{89}$ Darin begründet sich die Verknüpfung des semantischen Parameters der Produktivität mit demjenigen der Beschränkungen und Präferenzen, insbesondere mit Präferenzen für lexikalische Frames (Unterabschnitt 5.3.3). Diese von lexikalischen Frames ausgehende Generalisierung überträgt sich freilich auf die LE und lexikalischen Bedeutungen, die unter einem Frame zusammenzufassen sind: Ist ein lexikalischer Frame, der in einen KonstruktFrame eingeht, ,neu' und damit unrelatiert, so sind es auch die LE, die diesen Frame evozieren sowie deren lexikalische Bedeutungen. ${ }^{90}$

Unrelatierte lexikalische Frames sind nicht nur deshalb für die Produktivität einer Konstruktion entscheidend, weil sie im engeren Sinne Kennzeichen ,neuer‘ Instanzen der Konstruktion sind, sondern weil sie direkt den Einfluss des Konstruktions-Frames auf die Konstitution von Konstrukt-Frames zeigen und damit Ausdruck des Koerzionspotenzials der Konstruktion sind (vgl. Unterabschnitt 5.5.2). Konstrukte, bei denen unrelatierte lexikalische Frames in die Konstitution ihrer Konstrukt-Frames eingehen, beinhalten in jenen Konstrukt-Frames in der Regel immer einen Anteil des Konstruktions-Frames. Der Konstrukt-Frame kommt also, gemäß dem konstruktionssemantischen Modell (Kapitel 4) - und anders als

89 Theoretisch lässt sich die Aufnahme unrelatierter lexikalischer Frames in Konstrukt-Frames mit dem Begriff der Abweichung (exploitation) von Hanks (2011: 497-500, 2013: 211-250) in Verbindung bringen. Damit lässt sich erklären, warum unrelatierte lexikalische Frames, also solche ohne eine Frame-Nähe zum Konstruktions-Frame (Abschnitt 5.4) als besonders ,kreativ“ eingestuft werden können - denn: „Das Phänomen der Abweichung ist ein zentraler Aspekt der kreativen, dynamischen Natur von Sprache“ (Hanks 2011: 499). Expliziter diskutiert Bergs (2018: 283-285) Koerzionseffekte als eine Form von Kreativität, die Sampson (2016:17) E-Kreativität nennt, wobei das E für erweiternd steht und diese sich von der bereits erwähnten F-Kreativität in ihrer Innovationsstärke unterscheidet.

90 Herbst (2018a: 316) spricht hierfür auch von atypischen $L E$. Eine solche Form von lexikalischer Variation sieht Herbst (2018a: 319) als wesentliche Triebfeder von Kreativität an: „[L]inguistic creativity has a lot to do with lexical variation, in particular with using particular lexical units in constructions in which one would not expect them.“ 
bei Konstrukten mit relatierten lexikalischen Frames - als ein Blend aus lexikalischem Frame und Konstruktions-Frame und somit durch Frame-Anpassung zustande (vgl. Unterabschnitt 4.4.1), die deutlich voneinander getrennt sind, weil sie nicht in einer Frame-Nähe zueinander stehen. In allen Konstrukten mit unrelatierten lexikalischen Frames liegt somit Koerzion vor, weshalb diese für die Messung des Koerzionspotenzials der Konstruktion besonders interessant sind (vgl. Unterabschnitt 5.5.2). Dies ist die Art und Weise, in der der Parameter der Produktivität mit demjenigen des Koerzionspotenzials (Abschnitt 5.5) in Verbindung steht, wie es bereits in Abbildung $5.1 \mathrm{im}$ Eingang zu diesem Kapitel dargestellt ist. In den Worten von Suttle \& Goldberg (2011: 1238): „A construction is considered to be productive to the extent that it can coerce new words to appear in it." Das Koerzionspotenzial einer Konstruktion gibt nun Auskunft darüber, wie affin eine Konstruktion zur Bildung ,neuer' Konstrukte mit unrelatierten lexikalischen Frames ist. Da das Koerzionspotenzial insbesondere auf Präferenzen für lexikalische Frames (Unterabschnitt 5.3.3) aufbaut, ist auch der semantische Parameter der Produktivität mit demjenigen der Beschränkungen und Präferenzen verknüpft.

Die Menge der unrelatierten lexikalischen Frames und damit diejenige der Konstrukte, die zur Erweiterung einer Konstruktion führen, lässt sich durch die Höhe der Koerzionseffekte in diesen Konstrukten feindifferenzieren. Je höher der Koerzionseffekt in einem Konstrukt ist, desto stärker treibt dieses Konstrukt die ,Erweiterung“ der Konstruktion voran. Bevor ich in Unterabschnitt 7.4.1 und 7.4.3 eine Methode vorschlage, wie die Höhe eines Koerzionseffekts bestimmt werden kann - was Ausgangspunkt für die Messung des Koerzionspotenzials ist -, sei an zwei Belegen illustriert, wie sich die Produktivität einer Konstruktion an Konstrukten mit unrelatierten lexikalischen Frames äußern kann.

Ein für die reflexive Bewegungskonstruktion eher niedriger Koerzionseffekt liegt in dem bereits in Unterabschnitt 5.5.2 als (85) zitierten Konstrukt mit dem lexikalischen Frame Manipulate_into_doing vor, das in (90) noch einmal wiederholt ist. Konstrukte wie dieses liegen tendenziell näher an Konstrukten mit semantisch kohärenten lexikalischen Frames wie das in Unterabschnitt 5.6.1 unter (89) für den lexikalischen Frame Making_faces zitierte.

(90) Der Fabrikant breitete sein Schweigen wieder aus, der Offizier [Manipulate_ into_doing mogelte] sich aus seinem Sitz. (Düffel, John von: Vom Wasser, München: dtv 2006, S. 56)

Konstrukte wie das in (90) sind somit zwar Ausdruck der Erweiterung einer Konstruktion, sie sind aber aufgrund ihres niedrigen Koerzionseffekts näher an Konstrukten mit semantisch kohärenten lexikalischen Frames als etwa solche wie dasjenige in (91) mit dem lexikalischen Frame Dead_or_alive, das den für die reflexi- 
ve Bewegungskonstruktion höchstmöglichen Koerzionseffekt aufweist und damit eine deutliche Erweiterung der Konstruktion darstellt.

Man [Dead_or_alive lebte] sich aus der herausgerissenen Zeit, in der man saß, zurück in die Erinnerung ans Zuhause von damals und voraus in die Hoffnung, bald heimzukehren. (Müller, Herta: Der König verneigt sich und tötet, München: Carl Hanser Verlag 2003, S. 42)

Wie für die reflexive Bewegungskonstruktion kann für die beiden anderen Konstruktionen analog verfahren werden, um ihre Produktivität zu ermitteln. Dazu ist also nicht nur die in Unterabschnitt 5.6.1 erwähnte Type-Frequenz relatierter und damit semantisch kohärenter lexikalischer Frames einzubeziehen, sondern gleichzeitig jene unrelatierter lexikalischer Frames, die eine Erweiterung der Konstruktion um ,neue ' Konstrukte darstellen. Aufgrund dieser Erweiterung ist die Type-Frequenz unrelatierter lexikalischer Frames als relevanter als diejenige der relatierten lexikalischen Frames einzustufen. Dieser Punkt zeigt die Notwendigkeit für eine Methode zur Messung der Produktivität. Ich komme auf sie in Unterabschnitt 7.5.1 zurück und diskutiere die Ergebnisse für die drei untersuchten Konstruktionen in Unterabschnitt 7.5.2.

\subsection{Emergente Struktur}

Als Kerngegenstand des letzten der sieben semantischen Parameter von Konstruktionen möchte ich einen semantischen Aspekt diskutieren, der zunächst eine ,Restklasse“ darzustellen scheint, da es sich dabei auf den ersten Blick betrachtet um einen Bestandteil der semantischen Eigenschaften einer Konstruktion handelt, der sich nicht direkt aus den lexikalischen Frames oder dem KonstruktionsFrame zu ergeben und somit auch über die Annotation eines Konstrukts hinsichtlich seiner Strukturelemente und den FE, die sie semantisch motivieren, nicht erfassbar zu sein scheint. In den drei untersuchten Konstruktionen betrifft dies den Aspekt der ,Schwierigkeit' der ,Bewegung', die die durch das KtE des KE BEWEGENDES bezeichnete Entität vollzieht, auf den ich in Unterabschnitt 3.1.4 für die reflexive Bewegungskonstruktion hingewiesen habe. ${ }^{91}$ Auch solche Aspekte müssen Teil einer konstruktikographischen Beschreibung sein, da sie - ähnlich

91 Der Aspekt der ,Schwierigkeit“ findet sich auch in den Konstrukten der anderen beiden Konstruktionen, für die sich ebenso eine solche emergente Struktur feststellen lässt. Da diese in der bisherigen Forschung aber insbesondere für die reflexive Bewegungskonstruktion und noch stärker für die way-Konstruktion diskutiert worden ist, beschränke ich mich in der Beispielanalyse in 
wie das Koerzionspotenzial (Abschnitt 5.5) und die Produktivität (Abschnitt 5.6) einen nicht zu vernachlässigenden Teil der semantischen Leistung der Konstruktion ausmachen.

Bei genauerer Betrachtung stellt sich heraus, dass lexikalische Frames und der Konstruktions-Frame hinsichtlich der emergenten Struktur in einem Konstrukt zwar nicht spezifiziert sind, diese sich jedoch über den Einbezug von Standardwerten erklären lässt, welche für bestimmte FE des aus diesen beiden Frames resultierenden Konstrukt-Frames beobachtbar sind. Während ich bereits auf Standardwerte in lexikalischer Bedeutung und Konstruktionsbedeutung eingegangen bin (etwa in den Unterabschnitten 5.2.3 für die Differenzierung konstruktioneller Polysemie und in Unterabschnitt 5.2.4 und 5.5.2 für Koerzionseffekte), fehlt dies noch für die Konstruktbedeutung. Deshalb möchte ich zunächst in Unterabschnitt 5.7.1 skizzieren, welche Rolle Standardwerte für die Evokation einer emergenten Struktur spielen, um in Unterabschnitt 5.7.2 am Beispiel der reflexiven Bewegungskonstruktion drei sprachlich overte Phänomene zu diskutieren, die diese Standardwerte erkennen lassen.

\subsubsection{Standardwerte in Konstruktbedeutungen}

Wenngleich Konstrukt-Frames in der Regel durch eine Komposition von lexikalischem Frame und Konstruktions-Frame zustande kommen (vgl. Unterabschnitt 4.1.3), gilt dies für die Konstruktbedeutung, die auf ihm beruht, nicht zwangsläufig. Standardwerte, die eine Konstruktbedeutung gegenüber dem ihr zugrunde liegenden Konstrukt-Frame spezifizieren, können sich ergeben, ohne dass sie bereits in lexikalischer Bedeutung oder Konstruktionsbedeutung enthalten sein müssen. Dass sich Standardwerte einer lexikalischen Bedeutung durch die Konstruktionsbedeutung tilgen lassen, habe ich durch eine Analyse in Unterabschnitt 5.5 .2 bereits gezeigt. Wie aber wirken Standardwerte in einer Konstruktbedeutung, wenn sie weder in einer lexikalischen Bedeutung noch in der Konstruktionsbedeutung angelegt sind?

Begrifflich möchte ich solche Standardwerte unter Rückgriff auf einen Terminus der Theorie der konzeptuellen Integration von Fauconnier \& Turner (1998a,b, 2002) erfassen: der emergenten Struktur (vgl. schon Unterabschnitt 4.1.3). Während lexikalischer Frame und lexikalische Bedeutung sowie Konstruktions-Frame und Konstruktionsbedeutung, wie in Kapitel 4 erläutert, als Inputs eines Netzwerk konzeptueller Integration zu verstehen sind und der Konstruktions-Frame und die

diesem Kapitel auf die reflexive Bewegungskonstruktion. In Unterabschnitt 7.6.2 nehme ich einen Vergleich der emergenten Struktur für alle drei Konstruktionen vor. 
Konstruktbedeutung als Blend gedeutet werden können (vgl. Abbildung 4.3 in Unterabschnitt 4.1.3), ist die emergente Struktur ein Bestandteil einer konzeptuellen Integration, den ich bisher noch nicht berücksichtigt habe. Wie Fauconnier \& Turner (1998a: 144) argumentieren, ist sie bei der Entstehung eines Blends unmittelbar erwartbar: „Composition, completion, and elaboration lead to emergent structure in the blend; the blend contains structure that is not copied from the inputs." Die emergente Struktur ist somit ein Format zur Erfassung von Standardwerten, die nicht auf Type-Ebene, in lexikalischer Bedeutung oder Konstruktionsbedeutung, angelegt sein müssen: „The blend develops emergent structure that is not in the inputs." (Fauconnier \& Turner 2002: 42). ${ }^{92}$

Eine wesentliche Motivation, die Theorie der konzeptuellen Integration zur Erklärung der Konstitution eines Konstrukt-Frames und einer Konstruktbedeutung heranzuziehen, ist - neben der Tatsache, dass es sich bei einem Konstrukt-Frame in vielen Fällen um einen Blend aus lexikalischem Frame und Konstruktions-Frame handelt - das Vorhandensein von Standardwerten in Konstruktbedeutungen, die sich eben nicht isoliert entweder für den lexikalischen Frame oder den Konstruktions-Frame beobachten lassen, sondern die erst bei der Betrachtung des Konstrukt-Frames und damit der Gesamtheit eines Netzwerks konzeptueller Integration erfassbar werden.

Voraussetzung dafür, dass ein Standardwert in einem Konstrukt-Frame hin zu einer Konstruktbedeutung spezifiziert werden kann, ist, dass ein entsprechendes FE existiert, das diesen Standardwert trägt. Im Falle der reflexiven Bewegungskonstruktion und der emergenten Struktur, die in einer ,Schwierigkeit der ,Bewegung', die mit dem in einem Konstrukt ausgedrückten Ereignis einhergeht, besteht, handelt es sich dabei um ein FE MANNER, ${ }^{93}$ das entweder bereits im lexikalischen Frame enthalten ist oder durch den Konstruktions-Frame und dessen FE Motion.〈MANNER〉 im Konstrukt-Frame beigetragen wird. Das FE selbst ist somit noch kein Ausdruck der emergenten Struktur, sondern erst sein Standardwert, der innerhalb der Konstruktbedeutung entsteht. Auf drei Varianten, die diesen Stan-

92 Den Begriff der Emergenz verwende ich demnach nicht im radikal gebrauchsbasierten Sinne, wie dies etwa Hopper $(1987,1998,2011)$ tut (vgl. auch Auer \& Pfänder 2011), sondern gehe mit Traugott \& Trousdale (2013: 48) davon aus, dass Emergenz auch mit im sprachlichen Wissen verankerten Strukturen (eben: Konstruktionen und deren semantischen Eigenschaften) interagieren kann.

93 Sofern ich nicht ein konkretes FE eines konkreten Frames bezeichne, sondern generisch darauf verweise, stelle ich das FE MANNER ohne Winkelklammern da, obwohl es in der Mehrheit der Frames ein Nicht-Kern-FE ist. Wann immer ich mich auf sein Enthaltensein in einem diskutierten Frame beziehe, notiere ich es entsprechend seines FE-Typs. 
dardwert an die sprachliche Oberfläche treten lassen, möchte ich im folgenden Unterabschnitt 5.7.2 eingehen.

\subsubsection{Die ,Schwierigkeit‘ der reflexiven Bewegungskonstruktion}

Ein Beispiel für eine emergente Struktur lässt sich für die reflexiven Bewegungskonstruktion darin finden, dass die ,Schwierigkeit' der ,Bewegung', die in einer Konstruktbedeutung entsteht, scheinbar nicht über Standardwerte in lexikalischer Bedeutung und Konstruktionsbedeutung erklärbar ist. Ohne eine solche strukturelle Erklärung zu suchen, bemerkt auch Goldberg (1997), dass es Fälle von Konstrukten gibt, in denen

the meaning of the construction and the meaning of the verb are not simply composed in an additive or monotonic fashion. Instead, the meaning of the verb is integrated with the meaning of the construction, resulting in entailments that neither the verb or construction have independently. (Goldberg 1997: 392)

Im Rückgriff auf die Strukturelemente von Konstruktionen und Frames reformuliert heißt das: Teile der Konstruktbedeutung sind scheinbar nicht über die semantische Motivierung der vorliegenden KtE durch FE von lexikalischem Frame und Konstruktions-Frame erklärbar und scheinbar nicht overt sprachlich ausgedrückt. Der sprachlich nicht overte Ausdruck des Standardwerts, der für die emergente Struktur verantwortlich ist, betrifft jedoch wiederum nur einen Teil der Möglichkeiten, wie dieser in einer Konstruktbedeutung spezifiziert werden kann. Ich betone deshalb das Attribut des scheinbar sprachlich nicht overten Ausdrucks, da bei genauerer Betrachtung durchaus zu beobachten ist, dass der Standardwert, auf den die ,Schwierigkeit‘ zurückzuführen ist, sehr wohl overt sichtbar werden kann..$^{94}$

Ich möchte nun drei Varianten unterscheiden, in denen der Standardwert der ,Schwierigkeit' der ,Bewegung' in einer Konstruktbedeutung entstehen kann. ${ }^{95}$ Erstere Variante ist eine, in der gerade kein separates FE MANNER instanziiert wird.

94 Diese Annahme widerspricht freilich (z.T. pragmatisch orientierten) Ansätzen, die dafür argumentieren, dass wesentliche Aspekte einer Bedeutung gerade nicht an der sprachlichen Oberfläche ,ablesbar' sind (z.B. Fauconnier 1990; Langacker 1999: 46-48; von Polenz 2008: 298-327; Busse 2012: 42). Ich stelle damit nicht grundsätzlich infrage, dass alle Komponenten einer (Konstrukt-)Bedeutung auch sprachlich ausgedrückt werden müssen, ziehe aber aus Gründen der Objektivierbarkeit und der konstruktionssemantischen Operationalisierbarkeit die Frage nach solchen overten Ausdrücken derjenigen nach unausgedrückten Bedeutungsbestandteilen vor.

95 Szcześniak (2013: 168-169) kritisiert für die way-Konstruktion gar, dass der Aspekt der ,Schwierigkeit‘ nicht am KEE-Bestandteil way festgemacht werden kann, was er nach seiner An- 
Dies trifft auch auf zweitere Variante zu, jedoch ist der sprachliche Ausdruck dort nicht auf ein einzelnes Strukturelement des Konstrukts und damit eine Instanz des Konstrukt-Frames beschränkt. Letztere Variante schließlich besteht in einer direkten overten Instanziierung eines FE MANNER im Konstrukt-Frame. Im Folgenden seien die drei Varianten dargestellt, im Anschluss möchte ich sie anhand einiger Belege für die reflexive Bewegungskonstruktion illustrieren.

a) Der Standardwert ,Schwierigkeit' ist in der lexikalischen Bedeutung, der LE, durch die das KE EREIGNIS instanziiert wird, bereits angelegt (und nicht overt ausgedrückt). ${ }^{96}$

b) Der Standardwert ,Schwierigkeit‘ entsteht durch einen kollokationalen Zusammenhang zwischen der LE als KtE des KE EREIGNIS und einem anderen KtE, allen voran demjenigen des KE WEG bzw. $\langle$ WEG $\rangle{ }^{97}$

c) Der Standardwert ,Schwierigkeit" wird overt durch die Instanziierung eines (i.d.R. Nicht-Kern-)FE wie MANNER ausgedrückt, dessen Realisierung zwar außerhalb der für die Konstruktion obligatorischen Strukturelemente liegt, aber (wie bei den anderen beiden Varianten) entweder im lexikalischen Frame oder im Konstruktions-Frame - z.B. in Gestalt des FE Motion.〈MANNER〉 angelegt ist.

Ad a) Die lexikalischen Bedeutungen mancher LE tragen den Aspekt der ,Schwierigkeit' bereits in sich. Schon Goldberg (1995: 203-205) spekuliert für die wayKonstruktion, dass bestimmte LE umso eher für ihre Instanzen infrage kommen, wenn sie eine ,Schwierigkeit' der Bewegung inhärent zumindest implizieren können. Für die reflexive Bewegungskonstruktion lässt sich feststellen, dass auch sie LE als KtE des KE EREIGNIS zulässt, die den Aspekt der ,Schwierigkeit‘ bereits inhärent kodieren. Genauer: In deren lexikalischer Bedeutung ist ein FE MANNER der jeweiligen lexikalischen Frames, die sie evozieren, bereits

sicht aber sein sollte. Ich halte diese Analyse für zu streng, da sich Leistungen der Konstruktion, wie ich in den folgenden Analysen zu sehen, auch an anderem overt realisierten Material auf Konstruktebene zeigen können. Auf die Schlussfolgerung, die Szcześniak aus seiner Maxime zu ziehen scheint und die ich, auf der Basis meiner Argumentation, teile, komme ich zum Abschluss dieses Unterabschnitts zurück.

96 Vgl. Goldberg (1995: 205, 1996: 37-38) für eine ähnliche Beobachtung für die wayKonstruktion. Vgl. auch Proost $(2015:$ 167, 172) für den Befund, dass bestimmte Implikaturen in Konstrukten einer präpositionalen Ditransitivkonstruktion von lexikalischen Bedeutungen abhängen.

97 Eine ganz ähnliche Bedingung scheint Szcześniak (2013: 178) vorzuschweben, ,,by treating the difficulty reading as a pragmatic inference triggered by the impression that the path expressed by the construction is of a special kind." Vgl. auch Szcześniak (2014b: 177-178) für eine ausführlichere Analyse. 
mit einem Standardwert wie ,schwierig' besetzt. Dazu zählen LE wie kämpfen (fight.v), quälen (torment.v) und zwängen (squeeze.v), die die lexikalischen Frames Hostile_encounter (92), Cause_to_experience (93) und Manipulation (94) evozieren.

(92) a. Dieses Jahr [Hostile_encounter kämpft] sich der CC-AS also durch seine 19. Session. (Die Zeit, 20.01.2000, Nr. 4)

b. David [Hostile_encounter kämpfte] sich durch das überfüllte Lokal und reduzierte seinen Wortschatz auf: Moment, gleich, Sekunde, bin schon da und sofort. (Suter, Martin: Lila, Lila, Zürich: Diogenes 2004, S. 52)

c. Das von Uganda unterstützte MLC BEMBAs, mittlerweile eine der größten Rebellengruppen, kontrolliert weite Gebiete im Osten und Norden des Kongo und [Hostile_encounter kämpft] sich in Richtung der Hauptstadt Kinshasa vor. (Archiv der Gegenwart, 2001 [2000])

(93) a. „Ich sitze hier von morgens bis abends am Schreibtisch und [Cause_to_experience quäle] mich durch eine Akte nach der anderen. (Düffel, John von: Houwelandt, Köln: DuMont Literatur und Kunst Verlag 2004, S. 137)

b. Rolf Knieper [Cause_to_experience quält] sich im Jeep durch die Wüste Turkmenistans und trinkt Wodka in mongolischen Partykellern. (Die Zeit, 24.02.2000, Nr. 9)

c. Vom sonnigen unteren Centovalli aus steigt man über fette Feuersalamander durch Esskastanienwälder bergan, schwitzt, dampft, springt nackt in einen Bergbach, [cause_to_experience quält] sich über verrutschte Pfade an Ziegenvolk vorbei ins Baumlose\}, zieht einen Pullover an, wirft den ersten Schneeball, und bei 1000 Metern über null versinkt man bis zur Hüfte im Schnee. (Die Zeit, 30.03.2000, Nr. 14)

(94) a. Der Ministerpräsident [Manipulation zwängt] sich samt Tross durch die kahlen Betonkavernen, sieht die armen Betten dicht an dicht, dazu die Notmatratze. (Die Zeit, 13.04.2000, Nr. 16)

b. Im belgischen Eupen [Manipulation zwängen] sich die Gendarmen der Rijkswacht mit ihren Computern und Funkanlagen in die düsteren Kammern einer Kaserne anno 1900, klaglos übrigens, weil wenigstens technisch gut versorgt; im Hof stehen Streifenwagen, aber sonst sieht alles aus wie damals, als hier Pferde wieherten. (Die Zeit, 20.04.2000, Nr. 17) 
c. Sie legte das Klassenbuch auf den Lehrertisch und [Manipulation zwängte] sich, ohne ihren Stuhl zurückzuschieben, auf ihren Platz. (Schulze, Ingo: Neue Leben, Berlin: Berlin Verlag 2005, S. 759)

Die Hypothese, die Goldberg (1995: 205) für die way-Konstruktion aufstellt, nach welcher „monomorphemic (basic or superordinate level) motion verbs are typically unacceptable in this construction“, wobei die Begründung dafür lautet, dass "[t]hese vanilla motion verbs do not normally imply that there is any difficulty or indirect motion involved“ (Goldberg 1995: 205), gilt für die drei untersuchten Konstruktionen grundsätzlich nicht (vgl. für dasselbe Argument für die wayKonstruktion Luzondo Oyón 2013: 354-355; Szcześniak 2013: 177-178, 2014b: 163; Traugott \& Trousdale 2013: 88). ${ }^{98}$ Sie ist, wie ich bereits in Unterabschnitt 5.2.3 gezeigt habe, durchaus mit LE wie bewegen (move.v) oder begeben (go.v) belegt, die direkt Motion, also den Konstruktions-Frame, als lexikalischen Frame evozieren und als ,Bewegungsverben“ kaum basaler sein könnten. ${ }^{99}$ Allerdings hat Goldberg mit der Vermutung Recht, dass jene Konstrukte keine direkten Aspekte der ,Schwierigkeit' durch die LE kodieren, wie die folgenden Belege zu bewegen (95) und begeben (96) verdeutlichen sollen.

(95) a. Er [Motion bewegte] sich durch vermeintlich unauffällige harmonische Räume mit einer Sehnsucht nach Farbigkeit, die alle Grauwerte des Theoretischen löschte. (Die Zeit, 10.02.2000, Nr. 7)

b. Die von Beduinen geführte Karawane (bis zu 18 Teilnehmer) [Motion bewegt] sich in täglichen Etappen von vier bis sechs Stunden über Dünen und Sandfelder, durch die Wadis und Ebenen, wobei die Kamele als Reit- und Packtiere fungieren. (Die Zeit, 17.02.2000, Nr. 8)

c. Ein Drahtseil ist über den Fluß gespannt, mit einer beweglichen Winde ist das Fährboot daran festgetäut, und während die gewaltige Strömung auf das querstehende Ruder drückt, [Motion bewegen] sich Boot und Winde seitlich am Seil entlang von einem Ufer zum andern. (Düffel, John von: Vom Wasser, München: dtv 2006, S. 192)

(96) a. Ich befand mich gerade in München und [Motion begab] mich in ein Geschäft, das überaus luxuriös wirkte, wie ein Gourmettempel. (Die Zeit, 10.02.2000, Nr. 7)

98 In ihren diachronen Analysen differenzieren Mondorf (2011: 409) und Fanego (2019: 686) diese Beobachtung dahingehend, dass neutrale ,Bewegungsverben‘ im Laufe der Grammatikalisierung der way-Konstruktion weniger frequent wurden.

99 Ich komme auf diese Fälle bei der Diskussion der semantischen Motivierung der KtE in Abschnitt 6.2 zurück. 
b. Der Likud-Politiker SHARON [Motion begab] sich am 28. September mit einem großen Aufgebot an Sicherheitskräften auf den weiträumig abgesperrten Tempelberg, der den Felsendom und die Al-Aksa-Moschee, den drittheiligsten Ort der Moslems, umfasst. (Archiv der Gegenwart, 2001 [2000])

c. Am 10. Oktober [Motion begab] sich TSVANGIRAI freiwillig zu einem polizeilichen Verhör in Harare. (Archiv der Gegenwart, 2001 [2000])

Diese Befunde geben Anlass zu der Annahme, dass eine Kodierung der ,Schwierigkeit' als emergente Struktur über die lexikalische Bedeutung allein nur dann möglich ist, wenn diese einen entsprechenden Standardwert enthält. Sie verdeutlichen, warum der semantische Parameter der emergenten Struktur, wie in Abbildung 5.1 im Eingang zu diesem Kapitel dargestellt, mit dem Parameter des Koerzionspotenzials (Abschnitt 5.5) einerseits und mit dem der Produktivität (Abschnitt 5.6) andererseits zusammenhängen muss. Sind das Koerzionspotenzial und die Produktivität einer Konstruktion hoch, so ist zu erwarten, dass auch zahlreiche lexikalische Bedeutungen mit ihr auftreten können, die einen Standardwert wie ,schwierig' enthalten und somit die emergente Struktur evozieren. Allerdings trifft dieses Kriterium auf die lexikalischen Bedeutunggen von LE wie bewegen und begeben, wie ich bereits in Unterabschnitt 4.1.1 argumentiert habe, gerade nicht zu. Sollen sie innerhalb einer Konstruktbedeutung eine emergente Struktur aufweisen, müssen dafür andere Wege als die Spezifizierung eines Standardwerts in der lexikalischen Bedeutung existieren.

Ad b) Sofern die lexikalische Bedeutung allein noch nicht den Standardwert der ,Schwierigkeit‘ enthält, kann dieser entstehen, wenn die betreffende LE in Kollokation mit einem bestimmten KtE, insbesondere des KE WEG bzW. 〈WEG〉 steht. ${ }^{100}$ So kann etwa mit der LE arbeiten (work.v), das den lexikalischen Frame Work evoziert, nur dann eine emergente Struktur entstehen, wenn es, wie in den Belegen in (97), als Basiswort in Kollokationen zu Kollokatoren(phrasen) wie Bücherberge, 14 Pfund schweres Handbuch oder kompliziertes Kreuzworträt-

100 Auf solche Kollokationsrestriktionen weist auch Boas (2003a: 124-158) für die Lizenzierung von Resultativkonstruktionen hin. Szcześniak (2019b: 83, 88-92) zeigt für die way-Konstruktion und die zur reflexiven Bewegungskonstruktion strukturell parallele englische Reflexivkonstruktion durch Kollokationen hervorgerufende semantische und pragmatische Effekte auf. In einer breiteren, der Kollostruktionsanalyse angelehnten Definition von Kollokationen sieht Hilpert (2019: 20-22) Kollokationen gar als ein wesentliches Kriterium bei der Identifizierung von Konstruktionen an. Basierend auf einer solchen Kollostruktionsanalyse ließe sich die vorliegende Variante b der Evokation der emergenten Struktur mittels einer kovariierenden Kollexemanalyse (Gries \& Stefanowitsch 2004a; Stefanowitsch \& Gries 2005) untersuchen. 
sel steht. ${ }^{101}$ Ein bereits in der lexikalischen Bedeutung von arbeiten angelegter Standardwert ,schwierig‘ erscheint unplausibel.

(97) a. \{Ich [EREIGNis arbeite] mich [WEG durch Bücherberge] hindurch $\}$ und ziehe nach Stunden das Werk „Der Tod der Familie“ des - wie ich - dicken, leider schon toten Psychiaters David Cooper hervor, der den Ehrentitel „Anti-Psychiater“ tragen durfte. (Schuh, Franz: Schreibkräfte, Köln: DuMont 2000, S. 18)

b. \{[EREIGNis Arbeitete] sich [WeG durch ein 14 Pfund schweres Handbuch, das er vom FBI aus Washington erhalten hatte]\} (für die 70 Mark Luftfracht musste er seine Wirtin anpumpen), und schrieb ganz wie sein Vorbild Karl May über ein Land, das er nie mit eigenen Augen gesehen hatte. (Die Zeit, 06.04.2000, Nr. 15)

c. \{Er [EREIGNis arbeitete] sich [Weg durch ein kompliziertes Kreuzworträtsel]\}, hatte ein Bier aufgemacht und sich auf einen beschaulichen Abend eingestellt, als das Telefon läutete. (Glavinic, Thomas: Die Arbeit der Nacht, München Wien: Carl Hanser Verlag 2006, S. 381)

Da das FE 〈MANNER〉 sowohl im lexikalischen Frame Work als auch im Konstruktions-Frame Motion enthalten ist, fusionieren diese zu einem einzigen gleichnamigen FE des Konstrukt-Frames. Der Standardwert dieses FE ist nun als ,schwierig“ zu paraphrasieren, das FE selbst wird aber overt nicht realisiert, sondern der Standardwert entsteht lediglich durch die Kollokation mit bestimmten KtE des KE WEG bzW. 〈WEG〉.

Dieselben Beobachtungen lassen sich zu LE wie drängeln in (98) und schieben in (99) (beide push.v), die den lexikalischen Frame Cause_motion evozieren, mit Kollokatoren wie Menge oder Masse machen.

(98) a. \{Phillip [EREIGNIS drängelt] sich [WeG durch die Menge]\}. (Bach, Tamara: Marsmädchen, Hamburg: Verlag Friedrich Oetinger 2003, S. 132)

b. \{Katharina [EREIGNis drängelte] sich [WEG durch die Leute]\}. (Dölling, Beate: Hör auf zu trommeln, Herz, Weinheim: Beltz \& Gelberg 2003, S. 59)

(99) a. Laura ist ganz klein, sogar noch ein paar Zentimeter kleiner als ich, aber \{sie [EREIGNIs schiebt] sich [WEG durch die Menge]\} wie ein Türsteher. (Bach, Tamara: Marsmädchen, Hamburg: Verlag Friedrich Oetinger 2003, S. 59)

101 Die Begriffe Basis(wort) und Kollokator für die beiden Bestandteile einer Kollokation gehen auf den Kollokationsbegriff von Hausmann (1984: 401, 1985: 119) zurück. 
b. Auf einmal steigt von draußen der Stimmenpegel an, Fotografen hetzen herein, \{ein dichter Pulk dunkel gekleideter Männer [EREIGNis schiebt] sich wie ein Rammblock [WEG durch die Masse]\}. (Die Zeit, 16.03.2000, Nr. 12)

Interessant ist in diesem Zusammenhang auch die LE wühlen (rummage.v) mit dem lexikalischen Frame Scouring und Kollokatoren wie 1500 Seiten ärztlicher Diagnose, Bankbilanzen und das weiche Erdreich in den Belegen in (100). Auch für diese LE gilt, dass der Standardwert ,schwierig‘ erst für das im Konstrukt-Frame enthaltene FE MANNER spezifiziert wird und nicht bereits in der lexikalischen Bedeutung (und ebenso wenig in der Konstruktionsbedeutung) enthalten ist.

(100) a. $\quad$ Reporter wühlten sich [WEG durch 1500 Seiten ärztlicher Diagnose]\} und schenkten ihm dafür einen Freispruch ersten Grades mit Zitaten wie diesem: (Die Zeit, 20.01.2000, Nr. 4)

b. Von Jakarta bis Seoul - überall \{wühlen sich Wirtschaftsprüfer, Weltbanker und Experten des Internationalen Währungsfonds [WEG durch Bankbilanzen]\}. (Die Zeit, 17.02.2000, Nr. 8)

c. $\quad$ Lastwagen wühlen sich [WEg durch das weiche Erdreich]\}, kippen ockerbraunen Sand obenauf. (Die Zeit, 24.02.2000, Nr. 9)

Ad c) Können sowohl die lexikalische Bedeutung allein als auch entsprechende Kollokationsverhältnisse nicht für die Entstehung eines Standardwerts wie ,schwierig، in Anspruch genommen werden, kann ein FE MANNER des KonstruktFrames innerhalb des Konstrukts auch overt instanziiert werden und somit auf die ,Schwierigkeit‘ der ,Bewegung“, die mit dem Ereignis, das durch die LE ausgedrückt wird, einhergeht, hinweisen. Eine Fusion der beiden FE 〈MANNER〉 des lexikalischen Frames und des Konstruktions-Frames im Konstrukt-Frame ist hierfür nicht zwingend erforderlich, da bereits der Konstruktions-Frame Motion über das entsprechende FE Motion.〈MANNER〉 verfügt. Dieses FE gehört selbst in Konstrukten, deren Konstruktbedeutung durch lexikalische Bedeutung und Konstruktionsbedeutung gemeinsam konstituiert wird, nicht zur Motivierung der Instanzen der obligatorischen Strukturelemente. Deshalb liegt die emergente Struktur in diesen Fällen zwar außerhalb jener obligatorischen Strukturelemente im engeren Sinne, das für sie notwendige FE, das den Standardwert trägt, ist aber in mindestens dem Konstruktions-Frame, in vielen Fällen auch in dem lexikalischen Frame, angelegt.

In Beleg (101) weist etwa das adverbial gebrauchte Adjektiv tapfer als Instanz des FE 〈MANNER〉, das sowohl im lexikalischen Frame Ingestion (essen/eat.v) als FE Ingestion.(MANNER) als auch im Konstruktions-Frame als FE 
Motion.〈MANNER〉 angelegt ist, auf die ,Schwierigkeit' hin. Es ist also durch eine entsprechende Fusion der FE beider Frames im Konstrukt-Frame enthalten. ${ }^{102}$

Also [Ingestion aßen] sich Kohl und Rühe [(MANnER) tapfer] durch große Stücke Marzipantorte, unter der Aufsicht von mindestens 15 Kamerateams und unter den kritischen Blicken eines ganzen Schwarms von Reportern, die lauerten und hofften - worauf eigentlich? (Die Zeit, 27.01.2000, Nr. 5)

Ein anderes Beispiel ist der lexikalische Frame Cause_to_amalgamate mit LE wie mischen (mix.v). Der die emergente Struktur erzeugende Standardwert in der Konstruktbedeutung von (102) wird durch das adverbial gebrauchte Adjektiv müde, als Instanz des FE Cause_to_amalgamate.〈MANNER〉, im Konstrukt-Frame erzeugt.

Die Lichter der Stadt leuchten ins Zimmer, ein fahler Mond [Cause_to_amalgamate mischt] sich [〈MANNER〉 müde] in das blausilberne Geflacker hinein. (Venske, Regula: Marthes Vision, Frankfurt am Main: Eichborn Verlag 2006, S. 11)

Auch für den lexikalischen Frame Bringing mit einer LE wie bringen (bring.v) ist ein Beispiel belegt. In (103) sorgt das adverbial gebrauchte Adjektiv erschöpft für einen die emergente Struktur erzeugenden Standardwert.

Er [Bringing brachte] sich [〈MANNER erschöpft] in Seitenlage, wegen der Wunde. (Kopetzky, Steffen: Grand Tour, Frankfurt am Main: Eichborn 2002, S. 540)

Diese Variante des Ausdrucks der ,Schwierigkeit‘ offenbart darüber hinaus ein weiteres Detail: Nicht alle Konstruktbedeutungen der Konstrukte der reflexiven Bewegungskonstruktion enthalten diese emergente Struktur (vgl. schon Oya 1999: 364) und somit einen entsprechenden Standardwert. ${ }^{103}$ Dies ist daran erkennbar, dass bestimmte Instanzen des FE 〈MANNER〉 gleichermaßen auf das ,Gegenteil‘ einer ,Schwierigkeit‘ hinweisen können, gewissermaßen auf eine ,Einfachheit‘ der ,Bewegung‘. Um noch einmal auf den lexikalischen Frame Ingestion zurückzukommen, sei dafür auf die Belege in (104) verwiesen. So lassen Instanziierungen wie langsam, genügsam, manchmal mit wirklicher Anteilnahme oder unbarmherzig

102 Da das FE Motion.(MANNER) im Konstruktions-Frame ohnehin angelegt ist, stelle ich in den folgenden Annotationen lediglich die MANNER-FE der lexikalischen Frames dar. Für relatierte lexikalische Frames ist ohnehin nur die Annotation des FE dieses Frames relevant.

$103 \mathrm{Zu}$ einer solchen Schlussfolgerung kommt auch Proost (2015: 171-172) für bestimmte Implikaturen bei Ditransitivkonstruktionen. 
eher keine Interpretation einer ,Schwierigkeit‘ zu, sondern legen das Gegenteil nahe.

(104) a. Abends las ich, die Bücherei war ziemlich umfangreich, und ich [Ingestion fraß] mich durch alles; [〈MANNER〉 langsam, genügsam, manchmal mit wirklicher Anteilnahme]. (Schmitter, Elke: Frau Sartoris, Berlin: BvT 2000[2002], S. 35)

b. Die nämlich erreichte im 18. Jahrhundert mit einem aus Amerika kommenden Schiff die französische Küste und [Ingestion fraß] sich [(MANNER〉 unbarmherzig] durch die französischen Weinäcker. (Die Zeit, 30.03.2000, Nr. 14)

Noch deutlicher ist der Ausdruck des Gegenteils einer ,Schwierigkeit' in den folgenden Belegen mit der lexikalischen Bedeutung der LE legen (lay.v) des lexikalischen Frames Placing in (105) sowie der lexikalischen Bedeutung der LE begeben (go.v) mit Motion in (106). Die adverbial gebrauchten Adjektive singend und freiwillig lassen deutlicher die Interpretation einer, Einfachheit‘ der ,Bewegung` $\mathrm{zu}$.

(105) Bald machten britische Panzer sowjetischen Panzern Platz, und Major Tatarov bestellte sich bei Tante Klara ein Schaumbad und [placing legte] sich [〈MANNER〉 singend] in Haueisens Wanne. (Koneffke, Jan: Paul Schatz im Uhrenkasten, Köln: DuMont Buchverlag 2000, S. 235)

(106) Ein jeder von ihnen [motion begibt] sich [(MANNER\} freiwillig] in dieselbe Lage, in der auch die Teilnehmer sind, die er beobachtet. (Die Zeit, 09.03.2000, Nr. 11)

Dieser empirische Befund für die reflexive Bewegungskonstruktion lässt sich an dieser Stelle bereits generalisieren: Die emergente Struktur ist keine notwendige Eigenschaft einer Konstruktbedeutung. Am Beispiel der ,Schwierigkeit‘ ist dies bereits für die way-Konstruktion dokumentiert:

The difficulty reading arises frequently enough to be taken for a prototypical meaning component of the construction, but it is certainly not found in all uses and is not a necessary condition for a use to sound natural and grammatical. (Szcześniak 2013: 163)

Die reflexive Bewegungskonstruktion zeigt, dass selbst in Konstruktionen, zu deren Leistung eine emergente Struktur zählt, nicht jede Konstruktbedeutung von ihr erfasst werden muss. Die drei Varianten der möglichen Kodierung einer emergenten Struktur, die ich aufgezeigt habe, sind ein erster Maßstab, um zu überprüfen, ob eine emergente Struktur überhaupt vorliegt und wie sie über die Konstrukte der betreffenden Konstruktion verteilt ist. Neben dem Auftreten der drei Vari- 
anten, die die emergente Struktur erzeugen, müssen allerdings ebenso jene Fälle dokumentiert werden, in denen das ,Gegenteil‘ einer ,Schwierigkeit‘ vorliegt, sowie diejenigen, in denen weder das eine noch das andere auftritt, die sich also gegenüber der emergenten Struktur ,neutral' verhalten. Eine solche Dokumentation ermöglicht es, über die einzelnen Konstrukte einer Konstruktion hinweg zu einer Verteilung dieser insgesamt sieben unterschiedlichen Fälle (drei Varianten, deren jeweiliges ,Gegenteil‘ sowie die ,neutralen' Fälle) zu gelangen und diese als entsprechenden ,Wert' des semantischen Parameters der emergenten Struktur konstruktikographisch zu erfassen. Ich komme darauf in Abschnitt 7.6 zurück.

Die Tatsache, dass nicht alle Konstrukte der Konstruktion von einer emergenten Struktur wie der ,Schwierigkeit‘ der reflexiven Bewegungskonstruktion erfasst werden, macht zugleich deutlich, warum dieser Parameter nur im Rückgriff auf Konstruktbedeutungen analysiert werden und nicht wie viele andere Parameter lediglich mit lexikalischen Frames oder dem Konstruktions-Frame operieren kann, wie etwa Präferenzen für lexikalische Frames (Unterabschnitt 5.3.3), FrameNähe (Abschnitt 5.4), Koerzionspotenzial (Abschnitt 5.5) und Produktivität (Abschnitt 5.6). Somit gehört das von ihm erfasste Phänomen streng genommen nicht zu den invarianten semantischen Eigenschaften einer Konstruktion, die auf alle Konstrukte gleichermaßen zutreffen. Szcześniak (2013) argumentiert, dass es sich bei der ,Schwierigkeit‘ um eine Implikatur handeln muss, die als solche nicht als Bestandteil der semantischen Eigenschaften einer Konstruktion angesehen werden kann (vgl. auch Szcześniak 2014b: 162-163):104

If the difficulty reading is a defining property of the construction, it should be either an entailment or a presupposition. Implicatures are attributed to utterances, not specific linguistic forms, and they are usually secondary effects of other properties, and thus not part of the construction's meaning. (Szcześniak 2013: 165)

Selbst wenn es sich bei dem Aspekt der ,Schwierigkeit‘ um eine Implikatur handelt, steht diese einer Konventionalisierung und damit der Integration in die

104 An dieser Stelle wird der Bedarf für eine Diskussion um die Trennung zwischen Semantik und Pragmatik sichtbar, die ich hier allerdings nicht leisten kann. Wie bereits Lakoff (1987: 138139) und Langacker (1987: 154) lehnt auch Goldberg (1995: 7) eine Trennung grundsätzlich ab, was in einen weiten Begriff von ,Bedeutung der Konstruktionsgrammatik mündet (vgl. Fischer \& Stefanowitsch 2007: 9). Allerdings gibt es seit längerem Stimmen, die für eine Wiederaufnahme der Diskussion sprechen: Vgl. etwa Rostila (2007: 89-91) zu Argumenten für eine graduelle, aber dennoch aufrechtzuerhaltende Unterscheidung. Fried (2010: 98-99) problematisiert ähnlich den Übergang zwischen semantischen und pragmatischen Aspekten einer Konstruktion. Vgl. weiterhin Finkbeiner (2019) für einen Überblick über Desiderate und den Forschungsstand zur Rolle der Pragmatik in der Konstruktionsgrammatik. Auch Finkbeiner plädiert für eine scharfe Unterscheidung zwischen Semantik und Pragmatik. 
semantischen Eigenschaften, die zu einer Konstruktion gehören, nicht im Wege. So resümiert etwa Bybee (2013: 56), dass Implikaturen in Gestalt von Inferenzen sehr wohl den Status einer semantischen Eigenschaft auf Type-Ebene erreichen können. Auch Finkbeiner (2019: 182-183) plädiert für die Berücksichtigung konventionalisierter pragmatischer Aspekte in die Beschreibung von Konstruktionen. Aus dezidiert kognitionslinguistischer Perspektive argumentiert auch Schmid (2020) dafür, dass pragmatische Phänomene wie Implikaturen (vgl. dazu speziell Schmid 2020: 277-279) kognitiven Verfestigungen unterworfen sein können und sich somit auf Type-Ebene manifestieren können:

If pragmatic associations vary by definition from context to context, and if routinization is based, also by definition, on the recognition of similarity, then at first sight pragmatic associations do not seem to qualify for repetition-driven entrenchment. However, [...] the routinization of associations can operate over whatever different usage events have in common. This suggests that recurrent properties of usage events are eligible for entrenchment after all. (Schmid 2020: 269)

Doch auch wenn man sich der Auffassung der emergenten Struktur als Implikatur nicht anschließen möchte, lässt sich festhalten, dass eine emergente Struktur produktiv in ,neuen' Konstrukten einer Konstruktion evoziert werden kann:

If specific inferences commonly occur with a construction, their representation will be strengthened and eventually they can be activated automatically when the construction occurs, making them, in essence, part of the meaning of the construction. (Bybee 2010: 109)

Bybees, Finkbeiners und Schmids Argumentationen halte ich gerade aus gebrauchsbasierter Perspektive für sinnvoll, weshalb ich davon ausgehe, dass eine konstruktikographische Relevanz des Parameters der emergenten Struktur gegeben ist. ${ }^{105}$ Das Beispiel der reflexiven Bewegungskonstruktion zeigt, dass sie als Teil der semantischen Leistung der Konstruktion, wenn auch nicht über alle Konstrukte hinweg invariant, eine semantische Eigenschaft der Konstruktion ist, was der breite Forschungskanon, bereits zur way-Konstruktion und auch zur reflexiven Bewegungskonstruktion, widerspiegelt. Die empirischen Befunde, sie nicht in allen Konstrukten lokalisieren zu können, geben keinen Anlass dazu, sie vollständig zu ignorieren und somit nicht in die konstruktikographische Beschreibung aufzunehmen. ${ }^{106}$ Das Frequenzkriterium, das Bybee (2010: 109) implizit

105 Eine Weiterverfolgung dieses Parameters in pragmatischer Perspektive könnte ihn mit dem Begriff des pragmatischen Templates von Liedtke (2018) in Verbindung bringen, der, wie von Liedtke (2018: 127) selbst explizit erwähnt, an die Konstruktionsgrammatik anschließbar ist.

106 Das von Szcześniak (2014a: 137-138) vertretene Argument, der semantische Aspekt der ,Schwierigkeit‘ sei der way-Konstruktion abzusprechen, weil es außer dieser Konstruktion kei- 
erwähnt, spricht ebenso dagegen, eine emergente Struktur zu ignorieren: Es handelt sich dabei keineswegs um ein Einzelphänomen, sondern es ist, wie sich im empirischen Vergleich der drei untersuchten Konstruktionen in Unterabschnitt 7.6.2 zeigen wird, durchaus frequent belegt. Die Evokation einer emergenten Struktur kann über Kriterien wie die drei Varianten, die ich aufgezeigt habe, geleistet werden und bleibt dadurch fakultativ, aber dennoch ein wesentlicher Bestandteil der Konstruktion. Würde man die emergente Struktur ignorieren, wären etwa auch die Präferenzen für lexikalische Frames (Unterabschnitt 5.3.3) irrelevant, da auch sie ja nicht auf alle Konstrukte gleichermaßen zutreffen.

Selbst wenn man die Tatsache, dass sich eine emergente Struktur wie der semantische Aspekt der ,Schwierigkeit' nicht in allen Konstrukten der betreffenden Konstruktion finden lässt, nicht als Argument dafür gelten lässt, sie bei der Analyse zu ignorieren, stellt sich natürlich die Frage, ob sie überhaupt einer Konstruktion auf Type-Ebene zugeschrieben werden kann und damit einen eigenen semantischen Parameter von Konstruktionen rechtfertigt. Neben dem soeben diskutierten Aspekt der Konventionalisierung lässt sich Folgendes gegen einen solchen Vorbehalt einwenden: Obwohl die emergente Struktur allein als Standardwert innerhalb einer Konstruktbedeutung und damit auf Token-Ebene entsteht, ist sie dennoch charakteristisch für die betreffende Konstruktion. Dies wird dadurch deutlich, dass sich die ,Schwierigkeit“ im Falle der drei untersuchten Konstruktionen auf das Ereignis der ,Bewegung' bezieht und damit auf den Konstruktions-Frame oder einen relatierten lexikalischen Frame. Gerade bei unrelatierten lexikalischen Frames ist diese ,Schwierigkeit‘ ganz wesentlich an den Konstruktions-Frame gebunden, worauf sich die Varianten b und c der Evokation einer emergenten Struktur maßgeblich stützen. Variante b ist bei einem unrelatierten lexikalischen Frame insbesondere dann möglich, wenn das KtE des KE WEG bzw. 〈WEG〉 durch ein FE des Konstruktions-Frames semantisch motiviert wird, entweder gemeinsam mit einem FE des lexikalischen Frames (Unterabschnitt 6.2.3) oder allein durch ein FE des Konstruktions-Frames (Unterabschnitt 6.2.2). Wäre der Konstruktions-Frame an der semantischen Motivierung dieses KtE nicht beteiligt, könnte die emergente Struktur nicht durch diese Variante evoziert werden.

Ähnliches gilt für Variante c. Sollte der lexikalische Frame von sich aus kein FE wie MANNER beinhalten, durch das der Standardwert der ,Schwierigkeit‘ overt

ne anderen sprachlichen Formen gibt, ihn auszudrücken, wirkt vor diesem Hintergrund geradezu absurd: Selbst wenn es der Fall sein sollte, dass ein vergleichbarer semantischer Aspekt einer Konstruktbedeutung nirgendwo sonst in einer Sprache zu finden ist (wofür Szcześniak keine empirischen Belege liefert), wäre die unikale Eigenschaft der Konstruktion, ebendiesen semantischen Aspekt zu kodieren, gerade ein Argument, sie als semantisch besonders aussagekräftig anzusehen. 
ausgedrückt werden kann, tritt auch hier der Konstruktions-Frame ein und stellt ein FE wie Motion.(MANNER) zur Verfügung. Damit wird deutlich, dass der Standardwert der ,Schwierigkeit“ nicht nur charakteristisch für Konstrukte einer Konstruktion wie der reflexiven Bewegungskonstruktion ist, sondern dass dessen Evokation ganz gezielt auf den Konstruktions-Frame und seine FE zurückgeführt werden kann. Da der Konstruktions-Frame auf Type-Ebene liegt und mit der Konstruktion als Ganzes assoziiert ist (vgl. Unterabschnitt 4.1 und Abschnitt 4.3), ist die emergente Struktur, wie sie für die drei untersuchten Konstruktionen festgestellt werden kann, als Gegenstand eines semantischen Parameters von Konstruktionen (und damit ebenso auf Type-Ebene) zu verstehen und nicht allein ein Phänomen von Konstruktbedeutungen, wenngleich sie sich vordergründig dort offenbart.

Über diese Aspekte hinaus bietet die Analyse einer emergenten Struktur die Chance, über die reine Analyse der semantischen Motivierung der Strukturelemente von Konstrukten durch FE von lexikalischem Frame und KonstruktionsFrame hinauszugehen. Dieser Aspekt betrifft insbesondere die unter den Punkten a und b genannten Varianten der Evokation einer emergenten Struktur. Punkt c hingegen erlaubt die Erfassung der Instanzen von Strukturelementen, die außerhalb der obligatorischen Strukturelemente der Konstruktion liegen. Dass diese dennoch für die Konstruktbedeutung relevant sind, ist durch ihre Erzeugung einer emergenten Struktur evident. Sie lassen sich über diesen Parameter systematisch in die konstruktikographische Beschreibung integrieren. 